\title{
QUANTUM COHOMOLOGY AND TORIC MINIMAL MODEL PROGRAMS
}

\author{
EDUARDO GONZÁLEZ AND CHRIS T. WOODWARD
}

\begin{abstract}
We give a quantum version of the Danilov-Jurkiewicz presentation of the cohomology of a compact toric orbifold with projective coarse moduli space. More precisely, we construct a canonical isomorphism from a formal version of the Batyrev ring from [4] to the quantum orbifold cohomology at a canonical bulk deformation. This isomorphism generalizes results of Givental [23], Iritani [34] and Fukaya-Oh-Ohta-Ono [21] for toric manifolds and Coates-Lee-Corti-Tseng [11] for weighted projective spaces. The proof uses a quantum version of Kirwan surjectivity (Theorem 2.6 below) and an equality of dimensions (Theorem 4.19 below) deduced using a toric minimal model program (tmmp). We show that there is a natural decomposition of the quantum cohomology where summands correspond to singularities in the tmmp, each of which gives rise to a collection of Hamiltonian non-displaceable Lagrangian tori.
\end{abstract}

\section{Contents}

1. Introduction 1

2. Quantum Kirwan surjectivity for toric orbifolds $\quad 15$

3. Quantum Stanley-Reisner ring and Jacobian ring 21

4. Dimensional equality via a toric minimal model program 31

5. Minimal models and non-displaceable Lagrangians 44

References $\quad 48$

\section{INTRODUCTION}

According to results of Danilov and Jurkiewicz [16, 35, 36], the rational cohomology ring of a complete rationally-smooth toric variety is the quotient of a polynomial ring generated by prime invariant divisors by the Stanley-Reisner ideal. In addition to relations corresponding to linear equivalence of invariant divisors, there are higher degree relations corresponding to collections of divisors whose intersection is empty.

Partially supported by grants DMS1104670 and DMS0904358. We thank the Centre de Recerca Matemàtica, Barcelona, for hospitality during preparation of this paper.

Appeared in Adv. Math. Volume 353, 7 September 2019, Pages 591-646.
doi.org/10.1016/j.aim.2019.07.004. 
One can reformulate this presentation of the cohomology ring in terms of equivariant cohomology as follows. Let $G$ be a complex reductive group acting on a smooth polarized projective variety $X$. If the action on the semistable locus $X^{\mathrm{ss}}$ is locally free then the geometric invariant theory (git) quotient $X / / G=X^{\mathrm{ss}} / G$, by which we mean the stack-theoretic quotient of the semistable locus by the group action, is a smooth proper Deligne-Mumford stack with projective coarse moduli space. A result of Kirwan [38] says that the natural map $H_{G}(X, \mathbb{Q}) \rightarrow H(X / / G, \mathbb{Q})$ is surjective. Under suitable properness assumptions the same holds for quasi-projective $X$.

In particular, let $G$ be a torus acting on a finite-dimensional vector space $X$ with weights contained in an open half-space. The quotient $X / / G$ is a smooth proper Deligne-Mumford toric stack as in Borisov-Chen-Smith [7] and any such toric stack with projective coarse moduli space arises in this way. The equivariant cohomology $H_{G}(X)$ may be identified with the ring of polynomial functions on $\mathfrak{g}$ and each weight maps to a divisor class in $H(X / / G)$ under the Kirwan map. The Stanley-Reisner ideal $S R_{X}^{G}$ is precisely the kernel of the Kirwan map. For example, if $G=\mathbb{C}^{\times}$acts by scalar multiplication on $X=\mathbb{C}^{k}$, then $H_{G}(X)=\mathbb{Q}[\xi]$ is a polynomial ring in a single generator $\xi$, the git quotient is $X / / G=\mathbb{P}^{k-1}$, and the intersection of the $k$ prime invariant divisors is empty. The Stanley-Reisner ideal is the ideal $\left\langle\xi^{k}\right\rangle$ generated by $\xi^{k}$. This gives the standard description of the cohomology ring of projective space $H\left(\mathbb{P}^{k-1}\right)=H_{G}(X) / S R_{X}^{G}=\mathbb{Q}[\xi] /\left\langle\xi^{k}\right\rangle$.

In this paper we give a similar presentation of the quantum cohomology of compact toric orbifolds with projective coarse moduli spaces, via the quantum version of the Kirwan map introduced in $[50,51,52]$. The results here generalize those of Batyrev [4], Givental [23], Iritani [32, 33, 34], and Fukaya-Oh-Ohta-Ono [21], who use results of McDuff-Tolman [43]. In particular, Iritani [34] computed the quantum cohomology of toric manifolds using localization arguments for toric varieties that appear as certain complete intersections, while Fukaya et al [21] gave a computation using open-closed Gromov-Witten invariants defined via Kuranishi structures. The orbifold quantum cohomology of weighted projective spaces is computed in Coates-Lee-Corti-Tseng [11]. After the first version of this manuscript appeared a mirror theorem for toric stacks was proved by Coates, Corti, Iritani, and Tseng [12] and applied to give a Batyrev-style presentation in [13, Theorem 5.13].

A novel feature of the approach here is the appearance of minimal model programs, which are used to prove injectivity of the quantum Kirwan map modulo the quantum Stanley-Reisner ideal. The critical values of the Givental-Hori-Vafa potential acquire a natural geometric meaning in our approach: their logarithms are the transition times in the minimal model program, see Theorem 5.5 below, and the dimension of the orbifold cohomology and the logarithm of the lowest eigenvalue of quantum multiplication by the first Chern class decrease under each transition. We also obtain a more conceptual understanding of the appearance of open families of non-displaceable Lagrangians in toric orbifolds, as a consequence of the existence of infinitely many minimal model programs, see Remark 5.3.

We introduce the following notations. 
Notation 1.1. (a) (Novikov coefficients) Let $\Lambda$ denote the universal Novikov field of formal power series of $q$ with rational exponents

$$
\Lambda=\left\{\begin{array}{l|l}
\sum_{\rho} c_{\rho} q^{\rho} & \begin{array}{l}
c_{\rho} \in \mathbb{C}, \rho \in \mathbb{Q} \\
\forall e>0, \#\left\{\rho \mid c_{\rho}<e\right\}<\infty
\end{array}
\end{array}\right\}
$$

We denote by $\Lambda_{0} \subset \Lambda$ the subring with only non-negative powers of $q$.

(b) (Equivariant quantum cohomology) Let

$$
Q H_{G}(X):=H_{G}(X, \mathbb{C}) \otimes_{\mathbb{C}} \Lambda
$$

denote the (ungraded) equivariant quantum cohomology of $X$. We denote by $Q H_{G}(X, \mathbb{Q}):=H_{G}(X, \mathbb{Q}) \otimes_{\mathbb{Q}} \Lambda$ the subspace with rational coefficients. Equivariant enumeration of stable maps to $X$ defines a family of products

$$
\star_{\alpha}: T_{\alpha} Q H_{G}(X, \mathbb{Q})^{2} \rightarrow T_{\alpha} Q H_{G}(X, \mathbb{Q})
$$

forming (part of) the structure of a Frobenius manifold on $Q H_{G}(X, \mathbb{Q})[23]$ for $\alpha$ in a formal neighborhood of a symplectic class $\omega \in H_{2}^{G}(X, \mathbb{Q})$. Explicitly the product $\beta \star_{\alpha+\omega} \gamma$ is defined by

$$
\left\langle\beta \star_{\alpha+\omega} \gamma, \delta\right\rangle=\sum_{d \in H_{2}(X, \mathbb{Z}), n \geq 0} \frac{q^{\langle d, \omega\rangle}}{n !} \int_{\left[\overline{\mathcal{M}}_{0, n+3}(X, d)_{G}\right]} \operatorname{ev}^{*}\left(\alpha, \ldots, \alpha, \beta, \gamma, \delta^{\vee}\right)
$$

where the integral denotes push-forward to $B G$ using the equivariant virtual fundamental class described in [28].

(c) (Inertia stacks) The inertia stack of $X / / G$ is

$$
I_{X / / G}=\bigcup_{r>0} \operatorname{Hom}^{\mathrm{rep}}(\mathbb{P}(r), X / / G)=\bigcup_{[g]} X^{g, \mathrm{ss}} / Z_{g} .
$$

In the first union, $\operatorname{Hom}^{\mathrm{rep}}(\mathbb{P}(r), \cdot)$ denotes representable morphisms from $\mathbb{P}(r)=$ $B \mathbb{Z}_{r}$ and the second union is over conjugacy classes $[g]$ of elements $g \in G$, with $Z_{g} \subset G$ the centralizer of $g$ and $X^{g \text {,ss }}$ the intersection of the semistable locus $X^{\text {ss }}$ with the fixed point set

$$
X^{g}:=\{x \in X \mid g x=x\} .
$$

The rigidified inertia stack is

$$
\bar{I}_{X / / G}=\bigcup_{r>0} \operatorname{Hom}^{\mathrm{rep}}(\mathbb{P}(r), X / G) / \mathbb{P}(r)=\bigcup_{[g]} X^{g, \mathrm{ss}} /\left(Z_{g} /\langle g\rangle\right)
$$

where $\langle g\rangle$ denotes the subgroup generated by $g$, as in Abramovich-Graber-Vistoli [1], Chen-Ruan [10].

(d) (Orbifold quantum cohomology of a git quotient) Let

$$
Q H(X / / G):=H\left(I_{X / / G}, \mathbb{C}\right) \otimes \Lambda
$$

denote the orbifold quantum cohomology of $X / / G$, or $Q H(X / / G, \mathbb{Q})$ the version with rational coefficients. Enumeration of twisted stable maps to $X / / G$ (representable maps from orbifold curves to $X / / G$ ) defines a Frobenius manifold structure 
on $Q H(X / / G)[1],[10]$ given by a family of products

$$
\star_{\alpha}: T_{\alpha} Q H(X / / G, \mathbb{Q})^{2} \rightarrow T_{\alpha} Q H(X / / G, \mathbb{Q}) .
$$

These products are defined in a formal neighborhood of an equivariant symplectic class $\omega \in H^{2}(X / / G, \mathbb{Q})$ by

$$
\left\langle\beta \star_{\omega+\alpha} \gamma, \delta\right\rangle:=\sum_{\substack{d \in H_{2}(X / / G, \mathbb{Q}) \\ n \geq 0}} \frac{q^{\langle d, \omega\rangle}}{n !} \int_{\left[\overline{\mathcal{M}}_{0, n+3}(X / / G, d)\right]} \mathrm{ev}^{*}\left(\alpha, \ldots, \alpha, \beta, \gamma, \delta^{\vee}\right)
$$

for $\alpha, \beta, \gamma \in H\left(I_{X / / G}\right)$, extended by linearity over $\Lambda$. The pairing on the left-handside is a certain re-scaled Poincaré pairing on the inertia stack $I_{X / / G}$, see [1].

Example 1.2. To connect with the notation in [1], [10] (where one works with different Novikov fields) consider the following examples.

(a) (Stacky half-point) Let $G=\mathbb{C}^{\times}$act on $X=\mathbb{C}$ with weight two so that $X / / G=\mathbb{P}(2)$. The inertia stack $I_{X / / G}$ is the union of two copies of $\mathbb{P}(2)$ corresponding to the elements \pm 1 of $\mathbb{Z}_{2}$. Thus

$$
Q H(X / / G)=\Lambda \oplus \Lambda \theta_{-}
$$

the sum of two copies of $\Lambda$, where $\theta_{-}$is the additive generator of the twisted sector. Representable morphisms from a stacky curve $C$ to $X / / G=\mathbb{P}(2)$ correspond to double covers of the coarse moduli space $C$, with ramification at the stacky points. Since there is a unique double cover of the projective line with two ramification points (up to isomorphism) multiplication is given by $\theta_{-} \star_{\omega} \theta_{-}=1$.

(b) (Teardrop orbifold) Suppose that $G=\mathbb{C}^{\times}$acts on $X=\mathbb{C}^{2}$ with weights 1,2 . Then $X / / G=\mathbb{P}(1,2)$ is a weighted projective line, $Q H_{G}(X) \cong \Lambda[\xi]$ is a polynomial ring in a single generator, while

$$
Q H(X / / G)=\Lambda \oplus \Lambda \theta_{+} \oplus \Lambda \theta_{-}
$$

where $\theta_{+}$is the point class in $H(X / / G) \subset H\left(I_{X / / G}\right)$ and $\theta_{-}$is the class of the fixed point set $X^{-1} /\langle-1\rangle=\mathbb{P}(2)$ in the twisted sector. Identify $H_{2}^{G}(X, \mathbb{Q}) \cong \mathbb{Q}$ corresponding to the dual of the Euler class of the representation with weight one. The fundamental class in $H_{2}(X / / G, \mathbb{Q}) \cong H_{2}^{G}(X, \mathbb{Q})$ then maps to $1 / 2$. The moduli space of twisted stable maps $u: C \rightarrow \mathbb{P}(1,2)$ of genus and class zero is either isomorphic to $\mathbb{P}(1,2)$ for no stacky points in the domain $C$, or isomorphic to $\mathbb{P}(2)$, for two stacky points in the domain $C$. Furthermore there is a unique (up to isomorphism) homology class $1 / 2$ twisted map with two smooth marked points and one stacky marked point with $\mathbb{Z}_{2}$ automorphism group. It follows that if the symplectic class $\omega$ has area $1 / 2$ on the fundamental class of $\mathbb{P}(1,2)$ then the quantum product is defined by

$$
\theta_{+} \star_{\omega} \theta_{+}=q^{1 / 2} \theta_{-} / 2, \quad \theta_{-} \star_{\omega} \theta_{+}=q^{1 / 2} / 2, \quad \theta_{-} \star_{\omega} \theta_{-}=\theta_{+} .
$$

Thus after inverting $q^{1 / 2}$, the orbifold quantum cohomology is generated by $\theta_{+}$ with the relation $\theta_{+}^{3}=q / 4$. 
Remark 1.3. (Alternative power series rings) Some confusion may be caused by the multitude of formal power series rings that one can work over; unfortunately almost every set of authors has a different convention.

(a) The equivariant quantum cohomology $Q H_{G}(X)$ can be defined over the larger equivariant Novikov field $\Lambda_{X}^{G} \subset \operatorname{Map}\left(H_{2}^{G}(X, \mathbb{Z}), \mathbb{Q}\right)$ consisting of infinite sums $\sum_{i=1}^{\infty} c_{i} q^{d_{i}}$ with $\left\langle d_{i}, \omega\right\rangle \rightarrow \infty$, where $q^{d_{i}}$ is the delta function at $d_{i} \in H_{2}^{G}(X, \mathbb{Z})$. Similarly, the quantum cohomology of the quotient $Q H(X / / G)$ can be defined over the Novikov field $\Lambda_{X / / G} \subset \operatorname{Map}(H(X / / G, \mathbb{Q}), \mathbb{Q})$ consisting of infinite sums $\sum_{i=1}^{\infty} c_{i} q^{d_{i}}$ with $\left\langle d_{i}, \omega\right\rangle \rightarrow \infty$, where $q^{d_{i}}$ is the delta function at $d_{i} \in H_{2}(X / / G, \mathbb{Q})$. The advantage of these rings is that the equivariant quantum cohomology $Q H_{G}(X)$ becomes $\mathbb{Z}$-graded.

(b) $Q H_{G}(X)$ is also defined over the universal Novikov ring $\Lambda_{0}$. If $\omega$ is integral, then $Q H_{G}(X)$ is defined over $\mathbb{Q}[[q]]$. Similarly, $Q H(X / / G)$ is defined over the Novikov ring $\Lambda_{0}$, and if $\omega$ is integral, over $\mathbb{Q}\left[\left[q^{1 / n}\right]\right]$ for $n$ equal to the least common multiple of the orders of the automorphism groups in $X / / G$. However, it is convenient to work over the field $\Lambda$. Invariance under Hamiltonian perturbation only holds for Floer/quantum cohomology over the Novikov field $\Lambda$, and so working over $\Lambda$ is more natural for the purposes of symplectic geometry.

(c) Unfortunately, $\Lambda$ and $\Lambda_{0}$ are not finitely generated over $\mathbb{C}$ and so some care is required when talking about intersection multiplicities. In practice, when we wish to talk about intersection multiplicities we assume that the symplectic form is integral in which case our algebras are defined over $\mathbb{C}\left[q, q^{-1}\right]$.

(d) In algebraic geometry, one often uses the monoid-algebra of effective curve classes, but we prefer Novikov fields because of the better invariance properties. In fact, the cone of effective curve classes is not any more explicit than working over the Novikov field since it is the classes of connected curves that appear in the GromovWitten potentials, and these are rather hard to determine.

In $[50,51,52]$ the second author studied the relationship between $Q H_{G}(X)$ and $Q H(X / / G)$ given by virtual enumeration of affine gauged maps, called the quantum Kirwan map. An $n$-marked affine gauged map is a representable morphism from a weighted projective line $\mathbb{P}(1, r)$ for some $r>0$ to the quotient stack $X / G$ mapping $\mathbb{P}(r) \subset \mathbb{P}(1, r)$ to the semistable locus $X / / G$. Some of the results of $[50,51,52]$ are:

Theorem 1.4. (Definition and properties of the quantum Kirwan map)

(a) The stack $\mathcal{M}_{n, 1}^{G}(\mathbb{A}, X, d)$ of $n$-marked affine gauged maps of class $d \in H_{2}^{G}(X, \mathbb{Q})$ has a natural compactification $\overline{\mathcal{M}}_{n, 1}^{G}(\mathbb{A}, X, d)$. Denote by $\mathrm{ev}, \mathrm{ev}_{\infty}$ the evaluation maps

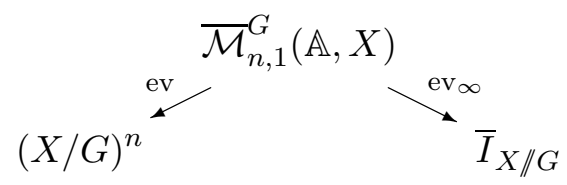


and $\mathrm{ev}_{d}, \mathrm{ev}_{d, \infty}$ their restrictions to maps of class $d$. The moduli stack $\overline{\mathcal{M}}_{n, 1}^{G}(\mathbb{A}, X, d)$ has a perfect relative obstruction theory over $\overline{\mathcal{M}}_{n, 1}(\mathbb{A})$ (the case of $X$ and $G$ trivial) where $\overline{\mathcal{M}}_{n, 1}(\mathbb{A})$ is the complexification of Stasheff's multiplihedron.

(b) For any $n \geq 0$, the map defined by virtual enumeration of stable $n$-marked affine gauged maps

$$
\kappa_{X}^{G, n}: Q H_{G}(X, \mathbb{Q}) \rightarrow Q H(X / / G, \mathbb{Q})
$$

$$
\alpha \mapsto \sum_{d \in H_{2}^{G}(X, \mathbb{Q})} q^{\langle d, \omega\rangle} \mathrm{ev}_{d, \infty, *} \operatorname{ev}_{d}^{*}(\alpha, \ldots, \alpha)
$$

is well-defined.

(c) The sum

$$
\kappa_{X}^{G}: Q H_{G}(X, \mathbb{Q}) \rightarrow Q H(X / / G, \mathbb{Q}), \quad \alpha \mapsto \sum_{n \geq 0} \frac{\kappa_{X}^{G, n}(\alpha)}{n !}
$$

defines a formal map from $Q H_{G}(X, \mathbb{Q})$ to $Q H(X / / G, \mathbb{Q})$ in a neighborhood of the symplectic class $\omega \in H_{G}^{2}(X, \mathbb{Q})$ with the property that each linearization

$$
D_{\alpha} \kappa_{X}^{G}: T_{\alpha} Q H_{G}(X, \mathbb{Q}) \rightarrow T_{\kappa_{X}^{G}(\alpha)} Q H(X / / G, \mathbb{Q})
$$

is a -homomorphism with respect to the quantum products.

By analogy with the classical case one hopes to obtain a presentation of the quantum cohomology algebra $T_{\kappa_{X}^{G}(\alpha)} Q H(X / / G, \mathbb{Q})$ by showing that $D_{\alpha} \kappa_{X}^{G}$ is surjective and computing its kernel. This hope leads to the following strong and weak quantum version of Kirwan surjectivity. In the strong form, one might hope that $\kappa_{X}^{G}$ has infinite radius of convergence, $\kappa_{X}^{G}$ is surjective, and $D_{\alpha} \kappa_{X}^{G}$ is surjective for any $\alpha \in Q H_{G}(X, \mathbb{Q})$. More modestly, one might hope that $D_{\alpha} \kappa_{X}^{G}$ is surjective for $\alpha$ in a formal neighborhood of a rational symplectic class $\omega \in H_{2}^{G}(X, \mathbb{Q})$.

We now specialize to the toric case. Suppose that $G$ is a complex torus with Lie algebra $\mathfrak{g}$ acting on a finite-dimensional complex vector space $X$.

Notation 1.5. (a) (Weights) Let $X_{1}, \ldots, X_{k} \subset X$ be the weight spaces of $X$ where $\operatorname{dim}\left(X_{j}\right)=1$ and $G$ acts on $X_{j}$ with weight $\mu_{j} \in \mathfrak{g}^{\vee}$ in the sense that for $x \in X_{j}$ and $\xi \in \mathfrak{g}$ we have $\exp (\xi) x=\exp \left(i\left\langle\xi, \mu_{j}\right\rangle\right), j=1, \ldots, k$. We assume that the weights $\mu_{j} \in \mathfrak{g}^{\vee}$ are contained in an open half-space, that is, for some $\nu \in \mathfrak{g}$ we have $\left\langle\nu, \mu_{i}\right\rangle \in \mathbb{R}_{>0}, i=1, \ldots, k$. We also assume that the weights $\mu_{i}$ span $\mathfrak{g}^{\vee}$, so that $G$ acts generically locally free on $X$.

(b) (Polarization and semistable locus) We assume that $X$ is equipped with a polarization, that is, an ample $G$-line bundle $L \rightarrow X$, which we may allow to be rational, that is, an integer root of an honest $G$-line bundle. Let $\omega \in \mathfrak{g}_{\mathbb{Q}}^{\vee}$ be the vector representing the first Chern class of the polarization $c_{1}^{G}(L) \in H_{2}^{G}(X, \mathbb{Q})$ under the isomorphism $\mathfrak{g}_{\mathbb{Q}}^{\vee} \cong H_{G}^{2}(X, \mathbb{Q})$. The point $\omega$ determines a rational polarization on 
$X$ with semistable locus given as follows. Let

$$
\mathcal{I}(\omega)=\left\{I \subset\{1, \ldots, k\} \mid \omega \notin \sum_{i \in I} \mathbb{R}_{\geq 0} \mu_{i}\right\}
$$

be the set of subsets so that $\omega$ is not in the span of the corresponding weights. Let $X^{I}$ be the intersection of coordinate hyperplanes

$$
X^{I}=\left\{\left(x_{1}, \ldots, x_{k}\right) \mid x_{i}=0, \forall i \notin I\right\} .
$$

Then

$$
X^{\mathrm{ss}}=X \backslash \bigcup_{I \in \mathcal{I}(\omega)} X^{I}
$$

The stable=semistable condition assumption translates to the condition for each $I \notin \mathcal{I}(\omega)$ the weights $\mu_{i}, i \in I$ span $\mathfrak{g}^{\vee}$. In this case the quotient $X / / G=X^{\mathrm{ss}} / G$ is then a smooth (possibly empty) proper Deligne-Mumford stack. We suppose that $X / / G$ is non-empty.

(c) (Quantum Stanley-Reisner ideal) The quantum Stanley-Reisner ideal is

$$
Q S R_{X, G}(\alpha):=\left\langle Q S R_{X, G}(d, \alpha), d \in H_{2}^{G}(X, \mathbb{Z})\right\rangle \subset Q H_{G}(X, \mathbb{Q})
$$

where

$$
Q S R_{X, G}(d, \alpha):=\prod_{\left\langle\mu_{j}, d\right\rangle \geq 0} \mu_{j}^{\left\langle\mu_{j}, d\right\rangle}-q^{\langle d, \alpha\rangle} \prod_{\left\langle\mu_{j}, d\right\rangle \leq 0} \mu_{j}^{-\left\langle\mu_{j}, d\right\rangle} .
$$

If $\alpha$ is the given symplectic class $\omega$, we write $Q S R_{X, G}:=Q S R_{X, G}(\omega)$. The quotient $T_{\omega} Q H_{G}(X, \mathbb{Q}) / Q S R_{X, G}$ is the quantum Stanley-Reisner a.k.a Batyrev ring.

Example 1.6. (a) (Batyrev ring for projective space) Let $G=\mathbb{C}^{\times}$act on $X=\mathbb{C}^{k}$ by scalar multiplication. All weights $\mu_{1}, \ldots, \mu_{k}$ are equal to $1 \in \mathfrak{g}_{\mathbb{Z}}^{\vee} \cong \mathbb{Z}$ and the polarization vector $\omega=1 \in \mathfrak{g}_{\mathbb{Q}}^{\vee} \cong H_{G}^{2}(X, \mathbb{Q})$. There is a unique subset $I=\emptyset$ in $\mathcal{I}(\omega)$ and $X^{I}=\{0\} \subset X$. Thus the semistable locus is $X^{\mathrm{ss}}=X-X^{\emptyset}=X-\{0\}$ and the git quotient is $X / / G=X^{\mathrm{ss}} / G=\mathbb{P}^{k-1}$. The quantum Stanley-Reisner ideal is generated by the single element $Q S R_{X, G}(1)=\xi^{k}-q$. The Batyrev ring is $\Lambda[\xi] /\left\langle\xi^{k}-q\right\rangle$.

(b) (Batyrev ring for the teardrop orbifold) Continuing Example 1.2 (b), suppose that $G=\mathbb{C}^{\times}$acts on $X=\mathbb{C}^{2}$ with weights 1,2 so that $X / / G=\mathbb{P}(1,2)$ is a weighted projective line. The Batyrev ring is $\Lambda[\xi] /\left\langle(\xi)(2 \xi)^{2}-q\right\rangle$.

(c) (Batyrev ring for the $B \mathbb{Z}_{2}$ ) Continuing Example 1.2 (b), suppose that $G=\mathbb{C}^{\times}$ acts on $X=\mathbb{C}$ with weights 2 so that $X / / G=\mathbb{P}(2) \cong B \mathbb{Z}_{2}$. The Batyrev ring is $\Lambda[\xi] /\left\langle(2 \xi)^{2}-q\right\rangle$. After specializing $q$, the Batyrev ring is isomorphic to the group ring of $\mathbb{Z}_{2}$.

Our main result says that Batyrev's original suggestion [4] for the quantum cohomology is true, after passing to a suitable formal version of the equivariant cohomology and "quantizing" the divisor classes: 
Theorem 1.7. For a suitable formal version $\widehat{Q H}_{G}(X)$ of the equivariant quantum cohomology $Q H_{G}(X)$ (see Section 2) the linearized quantum Kirwan map $D_{\omega} \kappa_{X}^{G}$ induces an isomorphism

$$
T_{\alpha} \widehat{Q H}_{G}(X, \mathbb{Q}) / \widehat{Q S R}_{X, G}(\omega) \rightarrow T_{\kappa_{X}^{G}(\omega)} Q H(X / / G, \mathbb{Q})
$$

at the tangent space to the rational symplectic class $\omega \in H_{G}^{2}(X, \mathbb{Q})$.

Remark 1.8. (a) Many earlier cases of this theorem were known. Batyrev [4] proved a similar presentation in the case of convex toric manifolds, that is, in the case that the deformations of any stable map are un-obstructed. In the semi-Fano case (that is, $c_{1}(X / / G)$ is non-negative on any curve class) a presentation was given by Givental [24]. For non-weak-Fano toric manifolds, Iritani [34, 5.11] gave an isomorphism with the Batyrev ring, see also Brown [8]. From the symplectic point of view a presentation for the quantum cohomology of toric manifolds was given in Fukaya et al [21], using results of McDuff-Tolman [43] on the Seidel representation. The latter approach uses open-closed Gromov-Witten invariants to define a potential counting holomorphic disks whose leading order terms are the potential above. The quantum Stanley-Reisner relations were proved by Coates, Corti, Iritani, and Tseng [13, Theorem 5.13], see also Woodward [50, 51, 52], in papers that appeared after the first version of this manuscript. That these relations generate the ideal was expected for some time, see Iritani [33]. Thus the main content of this paper is that these relations suffice. A quantization of the Borisov-Chen-Smith presentation of the orbifold cohomology [7] was given in Tseng-Wang [48]. The latter is not a presentation in terms of divisor classes; for example, for weighted projective spaces the typical number of generators is much larger than one, while the Batyrev ring has a single generator.

(b) For the result above to hold the quantum cohomology must be defined over the Novikov field, or at least, that a suitable rational power of the formal parameter $q$ has been inverted: over a polynomial ring such as $\mathbb{C}[q]$, one does not obtain an surjection because certain elements in twisted sectors are not contained in the image for $q=0$. Thus one sees a Batyrev presentation of the quantum cohomology only for non-zero $q$. The necessity of corrections to Batyrev's original conjecture, which involved the divisor classes as generators, was noted in Cox-Katz [14, Example 11.2.5.2] for the second Hirzebruch surface and Spielberg [46] for a toric threefold. The fact that the change of coordinates restores the original presentation was noted in Guest [30] for semi-Fano toric varieties, and Iritani [34, Section 5], for not-necessarily-Fano toric varieties in general, after passing to a formal completion. See Iritani [34, Example 5.5] and González-Iritani [26, Example 3.5] for examples in the toric manifold case.

(c) Note that Danilov's results [16] do not require projectivity of the coarse moduli space. It seems possible that quantum cohomology might also be defined for nonprojective toric varieties. Namely certain convergence conditions would remove the necessity of working over a Novikov ring, and one might have a theorem similar to 1.7 , but we lack any results in this direction. 
The presentation of the quantum cohomology in Theorem 1.7 can be re-phrased in terms of Landau-Ginzburg potential as follows, according to suggestions of Givental [23] and the physicists related to mirror symmetry. This formulation will be essential in our proof of the injectivity of the map in Theorem 1.7.

Notation 1.9. (a) (Residual torus) Let $\tilde{G}:=\left(\mathbb{C}^{\times}\right)^{k}$ denote the "big torus" act on $X=\mathbb{C}^{k}$ in the standard way. The residual torus

$$
T:=\tilde{G} / G
$$

has an induced action on $X / / G$. The Lie algebra $\mathfrak{t}$ of $T$ admits a canonical splitting into real and imaginary parts $\operatorname{Re} \oplus \operatorname{Im}: \mathfrak{t} \rightarrow \mathfrak{t}_{\mathbb{R}} \oplus i \mathfrak{t}_{\mathbb{R}}$. Let $T_{\mathbb{R}} \subset T$ denote the unitary part of $T$

$$
T_{\mathbb{R}}=\exp \left(\mathfrak{t}_{\mathbb{R}}\right), \quad \mathfrak{t}_{\mathbb{R}}=\operatorname{span}_{\mathbb{R}} \mathfrak{t}_{\mathbb{Z}}, \quad \mathfrak{t}_{\mathbb{Z}}=\exp ^{-1}(1)
$$

given by exponentiating the real span $\mathfrak{t}_{\mathbb{R}}$ of the coweights $\mathfrak{t}_{\mathbb{Z}}$ of $T$. We have an exact sequence of Lie algebras resp. finitely generated abelian groups

$$
0 \rightarrow \mathfrak{g} \rightarrow \tilde{\mathfrak{g}} \rightarrow \mathfrak{t} \rightarrow 0, \quad 0 \rightarrow \mathfrak{g}_{\mathbb{Z}} \rightarrow \tilde{\mathfrak{g}}_{\mathbb{Z}} \rightarrow \tilde{\mathfrak{t}}_{\mathbb{Z}} \rightarrow 0
$$

where $\tilde{\mathfrak{t}}_{\mathbb{Z}}:=\tilde{\mathfrak{g}}_{\mathbb{Z}} / \mathfrak{g}_{\mathbb{Z}}$. We write $\tilde{\mathfrak{t}}_{\mathbb{Z}}$ as the product of a free part $\mathfrak{t}_{\mathbb{Z}}$ of $\tilde{\mathfrak{t}}_{\mathbb{Z}}$ which is a lattice in $\mathfrak{t}$, and a torsion part $\Gamma$ which is isomorphic to the generic stabilizer of $G$ on $X$. A canonical parametrization of the residual torus $T=\tilde{G} / G$ can be found by row-reduction on the matrix of weights, see Example 1.11 below.

(b) (Moment polytope) The action of $T_{\mathbb{R}}$ on $X / / G$ is Hamiltonian, with moment map $\Phi: X / / G \rightarrow \mathfrak{t}_{\mathbb{R}}^{\vee}$ induced by the choice of moment map for the action of $\tilde{G}_{\mathbb{R}}=U(1)^{k}$ on $X$. Let $\Delta_{X / / G} \subset \mathfrak{t}_{\mathbb{R}}^{\vee}$ denote its image

$$
\Delta_{X / / G}:=\Phi(X / / G)
$$

the moment polytope of $X / / G$.

(c) (Facets and spurious inequalities) Let $\nu_{j} \in \mathfrak{t}_{\mathbb{Z}}, j=1, \ldots, k$ be the inward normal vectors to the facets of $\Delta_{X / / G}$; these are the images of minus the standard basis vectors $e_{j}$ of $\tilde{\mathfrak{g}}_{\mathbb{R}} \cong \mathbb{R}^{k}$ under the projection $\pi_{\mathfrak{t}}$ to $\mathfrak{t}_{\mathbb{R}}$ :

$$
\nu_{j}=\pi_{\mathfrak{t}}\left(-e_{j}\right), j=1, \ldots, k .
$$

The moment polytope $\Delta_{X / / G}$ is of the form

$$
\Delta_{X / / G}=\left\{\mu \in \mathfrak{t}_{\mathbb{R}}^{\vee} \mid\left\langle\mu, \nu_{j}\right\rangle \geq-\omega_{j}, j=1, \ldots, k\right\}
$$

with positions of the facets determined by elements $-\omega_{j} \in \mathbb{Q}$. We say that $\left\langle\mu, \nu_{j}\right\rangle \geq$ $-\omega_{j}$ is a spurious inequality if it does not correspond to a facet of $\Delta_{X / / G}$.

(d) (Support constants) The support constants $\omega_{j}$ defining the positions of the possible facets $F_{1}, \ldots, F_{k}$ of $\Delta_{X / / G}$ can be chosen as follows. Given an extension of $\omega$ to $H_{\tilde{G}}^{2}(X, \mathbb{Q}) \cong \mathbb{Q}^{k}$, the constants $\omega_{j}$ are the coefficients of $\omega$.

(e) (Dual torus) Recall from Iritani [33, Section 3] that the Landau-Ginzburg potential for toric orbifolds has domain a certain formal version of a finite cover of the dual torus $T^{\vee}$ to $T$. Define

$$
\begin{aligned}
& T^{\vee}=\operatorname{Hom}\left(\mathfrak{t}_{\mathbb{Z}}, \mathbb{C}^{\times}\right) \quad \tilde{T}^{\vee}=\operatorname{Hom}\left(\tilde{\mathfrak{t}}_{\mathbb{Z}}, \mathbb{C}^{\times}\right) \\
& G^{\vee}=\operatorname{Hom}\left(\mathfrak{g}_{\mathbb{Z}}, \mathbb{C}^{\times}\right) \quad \tilde{G}^{\vee}=\operatorname{Hom}\left(\tilde{\mathfrak{g}}_{\mathbb{Z}}^{k}, \mathbb{C}^{\times}\right) .
\end{aligned}
$$


We have $T^{\vee}=\tilde{T}^{\vee} / \Gamma$. Dualizing (5) gives a short exact sequence

$$
0 \rightarrow \tilde{T}^{\vee} \rightarrow \tilde{G}^{\vee} \rightarrow G^{\vee} \rightarrow 0 .
$$

In particular $\tilde{T}^{\vee}$ becomes a subgroup of $\tilde{G}^{\vee} \cong\left(\mathbb{C}^{\times}\right)^{k}$. Define the dual group over $\Lambda$

$$
\tilde{T}^{\vee}(\Lambda):=\operatorname{Hom}\left(\tilde{\mathfrak{t}}_{\mathbb{Z}}, \Lambda-\{0\}\right) .
$$

and similarly for $\tilde{G}^{\vee}(\Lambda)$. Define an injection

$$
\iota_{\omega}: \tilde{T}^{\vee}(\Lambda) \rightarrow \tilde{G}^{\vee}(\Lambda), \quad \tilde{g} \mapsto\left(q^{\omega_{1}}, \ldots, q^{\omega_{k}}\right) \tilde{g}
$$

that we call the quantum embedding of $\tilde{T}^{\vee}$. (The map $\iota_{\omega}$ is not a homomorphism.)

(f) (Givental potential) In the case of trivial generic stabilizer, the naive LandauGinzburg potential associated to the toric stack $X / / G$ is the function on the dual torus given as a sum of monomials whose exponents are the normal vectors to the facets of $\Delta_{X / / G}$ with coefficients $q^{\omega_{j}}$ :

$$
W_{X, G}: \tilde{T}^{\vee}(\Lambda) \rightarrow \Lambda, \quad y \mapsto \sum_{j=1}^{k} q^{\omega_{j}} y^{\nu_{j}}
$$

More generally, in the case of not-necessarily trivial generic stabilizer let $W_{X, G}$ denote the restriction of the function $\tilde{g}_{1}+\ldots+\tilde{g}_{k}$ to the subset $\iota_{\omega} \tilde{T}^{\vee} \subset \tilde{G}^{\vee}$. The reader may wish to compare with the definition of potential in Fukaya et al [21, Definition 2.1], where the potential is an element of a completed power series ring in coordinates $y_{j}^{ \pm}, j=1, \ldots, \operatorname{dim}(T)$. It was first noticed by Givental [25] that this function is related to the Gromov-Witten theory of $X / / G$. An explanation from the point of view of mirror symmetry was given in Hori-Vafa [31], and a connection to Floer theory is described in Fukaya et al [20]. In the latter the potential appears as a count of holomorphic disks with boundary in a fiber of the moment map. In the later version, the potential receives corrections from nodal holomorphic disks, whereas in Givental [25] and Hori-Vafa [31] there are no corrections.

Remark 1.10. (a) (Elimination of negative powers of $q$ ) As it stands, the values of $W_{X, G}$ have negative powers of $q$. However, later we will always assume that 0 is contained in the interior of the moment polytope $\Delta_{X / / G}$. In this case only positive powers $q^{\omega_{j}}$ of $q$ occur as coefficients in $W_{X / / G}$.

(b) (Naive small potential versus corrected small potential) For the many purposes (non-displaceability, Batyrev presentation) it seems that the naive potential is "as good as" the corrected potential defined by disk counts in Fukaya et all [20]. A heuristic argument that the two potentials are related by a geometrically-defined change of coordinates was given in Woodward [53]; for semi-Fano cases it is proved in Chan et al [9] that this coordinate change is the mirror map from GromovWitten theory, while Fukaya et al [21, Theorem 11.1] show the existence of some coordinate transformation relating the two. An approach to relating the potentials using an open version of the quantum Kirwan map is described in Woodward-Xu [54]. 
Example 1.11. (a) (Product of projective lines) Let $X=\mathbb{C}^{4}$ and $G=\left(\mathbb{C}^{\times}\right)^{2}$ with weights $\mu_{1}=(1,0), \mu_{2}=(1,0), \mu_{3}=(0,1), \mu_{4}=(0,1)$ and polarization vector $\omega=(0,1,0,1)$. The git quotient is $X / / G=\mathbb{P}^{1} \times \mathbb{P}^{1}$. The perpendicular space to the weights is found by row-reduction to be the span of the vectors $(1,-1,0,0),(0,0,1,-1)$. With the corresponding parametrization of the dual torus $T=\left(\mathbb{C}^{\times}\right)^{4} / G \cong\left(\mathbb{C}^{\times}\right)^{2}$ the normal vectors to the facets $F_{1}, F_{2}, F_{3}, F_{4}$ are

$$
\nu_{1}=(1,0), \nu_{2}=(-1,0), \nu_{3}=(0,1), \nu_{4}=(0,-1) .
$$

The moment polytope is $\Delta_{X / / G}=[0,1]^{2}$. The potential is

$$
W_{X, G}\left(y_{1}, y_{2}\right)=y_{1}+q / y_{1}+y_{2}+q / y_{2} \text {. }
$$

(b) (Projective line with extra term) The quotient of $X=\mathbb{C}^{3}$ by the action of $G=$ $\left(\mathbb{C}^{\times}\right)^{2}$ with weights $(1,0),(1,1),(-1,1)$ and polarization vector $(3,0,1)$ (which projects to $\left.(2,1) \in \mathfrak{g}^{\vee}\right)$ has semistable locus

$$
X^{\mathrm{ss}}=\left\{\left(x_{1}, x_{2}, x_{3}\right), x_{1} \neq 0,\left(x_{2}, x_{3}\right) \neq 0\right\}
$$

and git quotient $X / / G \cong \mathbb{P}^{1}$. The residual torus $T$ has Lie algebra $\mathfrak{t}$ identified with the span of $(-2,1,-1)$ in $\tilde{\mathfrak{g}}$. The moment polytope is

$$
\Delta_{X / / G}=\{\mu \in \mathbb{R} \mid 2 \mu \leq 3, \mu \geq 0, \mu \leq 1\} .
$$

The first inequality $2 \mu \leq 3$ is spurious, that is, may be removed without changing $\Delta_{X / / G}$. The potential is

$$
W_{X, G}(y)=q^{3} / y^{2}+y+q / y .
$$

(c) (Stacky half-point) Let $X=\mathbb{C}$ with weight $\mu_{1}=2$ so that $X / / G=\mathbb{P}(2) \cong B \mathbb{Z}_{2}$. Then $\tilde{T} \cong \mathbb{Z}_{2}$ and the embedding $\tilde{T}^{\vee} \rightarrow \tilde{G}^{\vee}$ is the standard one with image $\{ \pm 1\} \subset \tilde{G}^{\vee}$. The potential is then the isomorphism

$$
W_{X, G}: \tilde{T}^{\vee}(\Lambda) \cong \mathbb{Z}_{2} \rightarrow\{ \pm 1\} \subset \Lambda .
$$

Definition 1.12. (Critical locus and Jacobian ring) The critical locus $\operatorname{Crit}\left(W_{X, G}\right)$ of $W_{X, G}$ is the set of points with vanishing logarithmic derivatives with respect to the coordinates on $T^{\vee}$,

$$
\begin{aligned}
\operatorname{Crit}\left(W_{X, G}\right) & =\left\{y \in \tilde{T}^{\vee}(\Lambda)\left|\partial_{\lambda} W_{X, G}\left(y e^{\lambda}\right)\right|_{\lambda=0}=0 \quad \forall \lambda \in \mathfrak{t}_{\mathbb{R}}^{\vee}\right\} \\
& =\left\{y \in \tilde{T}^{\vee}(\Lambda) \mid \sum_{i=1}^{k}\left\langle\nu_{i}, \lambda\right\rangle q^{\omega_{i}} y^{\nu_{i}}=0, \quad \forall \lambda \in \mathfrak{t}_{\mathbb{R}}^{\vee}\right\} .
\end{aligned}
$$

Define the ring of functions on $\tilde{T}^{\vee}$

$$
\Lambda\left(\tilde{T}^{\vee}\right)=\bigoplus_{\lambda \in \tilde{\mathfrak{t}}_{\mathbb{Z}}} \Lambda y^{\lambda} .
$$

The ideal generated by the logarithmic partial derivatives of the potential is

$$
\left\langle\left.\partial_{\lambda} W_{X, G}\left(y e^{\lambda}\right)\right|_{\lambda=0}, \quad \lambda \in \mathfrak{t}_{\mathbb{R}}\right\rangle \subset \Lambda\left(\tilde{T}^{\vee}\right) .
$$


The Jacobian ring $\operatorname{Jac}\left(W_{X, G}\right)$ of the Givental potential $W_{X, G}$ is the ring of functions on $\operatorname{Crit}\left(W_{X, G}\right)$, or more precisely the quotient

$$
\operatorname{Jac}\left(W_{X, G}\right)=\Lambda\left(\tilde{T}^{\vee}\right) /\left\langle\partial_{\lambda} W_{X, G}\left(y e^{\lambda}\right)_{\lambda=0}\right\rangle
$$

If the generic stabilizer is trivial then we have equivalently using the notation (8)

$$
\operatorname{Jac}\left(W_{X, G}\right)=\Lambda\left[y^{ \pm \nu_{1}}, \ldots, y^{ \pm \nu_{k}}\right] /\left\langle y_{i} \partial_{y_{i}} W_{X, G}\right\rangle .
$$

Assuming that 0 is contained in the interior of the moment polytope and $\omega$ is integral then the potential $W_{X, G}$ is defined over $\mathbb{C}[q]$.

We wish to define a certain "positive part" of Crit $\left(W_{X, G}\right)$ whose coordinate ring corresponds to the quantum cohomology of $X / / G$.

Definition 1.13. (a) (Positive part of the Jacobian ring) Let $\mathcal{J} \subset \operatorname{Jac}\left(W_{X, G}\right)$ denote the ideal generated by the elements $q^{\omega_{j}} y_{j}, j=1, \ldots, k$, and $\widehat{\operatorname{Jac}}\left(W_{X, G}\right)$ the completion of $\operatorname{Jac}\left(W_{X, G}\right)$ with respect to $\mathcal{J}$,

$$
\widehat{\operatorname{Jac}}\left(W_{X, G}\right):={\underset{m}{\lim } \operatorname{Jac}} \operatorname{S}\left(W_{X, G}\right) / \mathcal{J}^{m} \text {. }
$$

Let $\mathrm{Jac}_{+}\left(W_{X, G}\right)$ denote the ring obtained from the formal completion by inverting $q$,

$$
\operatorname{Jac}_{+}\left(W_{X, G}\right):=\widehat{\operatorname{Jac}}\left(W_{X, G}\right)\left[q^{-1}\right] .
$$

(b) (Positive part of the critical locus) The filtered rings $\widehat{\operatorname{Jac}}\left(W_{X, G}\right)$ respectively $\mathrm{Jac}_{+}\left(W_{X, G}\right)$ are the ring of functions on the formal scheme $\widehat{\operatorname{Crit}}\left(W_{X, G}\right)$ resp. Crit $+\left(W_{X, G}\right)$ obtained by taking a formal neighborhood of $(y, q)=(0,0)$ in the closure of $\operatorname{Crit}\left(W_{X, G}\right)$ with respect to the embedding (7) resp. and removing the fiber over $q=0$. The scheme $\operatorname{Crit}_{+}\left(W_{X, G}\right)$ represents the locus of critical points $y(q)$ of $W_{X, G}$ that have limit $y(q) \rightarrow 0$ as $q \rightarrow 0$ with respect to the injection (7), that is, each expression $q^{\omega_{j}} y_{j}, j=1, \ldots, k$ has only positive powers of $q$. After passing to a cover $\operatorname{Spec} \mathbb{C}\left[q^{1 / n}, q^{-1}\right] \rightarrow \operatorname{Spec} \mathbb{C}\left[q, q^{-1}\right]$ for some $n$ we may write each solution near $q=0$ as a function $y(q)$ of a variable $q^{1 / n}$, that is, each component of $\operatorname{Crit}\left(W_{X, G}\right)$ becomes unramified over Spec $\mathbb{C}\left[q, q^{-1}\right]$. By a simple case of the Grothendieck Existence Theorem [29], any point of $\mathrm{Crit}_{+}\left(W_{X, G}\right)$ is obtained by completion and removing the locus with $q=0$ from a point of $\operatorname{Crit}\left(W_{X, G}\right)$ containing $(y, q)=(0,0)$. This ends the Definition.

Example 1.14. (a) (Critical locus for a product of projective lines) Continuing the example of a product of projective lines $X / / G=\mathbb{P}^{1} \times \mathbb{P}^{1}$ from Example 1.11 (a), the critical locus is defined by

$$
\begin{aligned}
& 0=y_{1} \partial_{y_{1}} W_{X, G}\left(y_{1}, y_{2}\right)=y_{1}-q / y_{1} \\
& 0=y_{2} \partial_{y_{2}} W_{X, G}\left(y_{1}, y_{2}\right)=y_{2}-q / y_{2} .
\end{aligned}
$$

The solutions are $\left(y_{1}, y_{2}\right)=( \pm \sqrt{q}, \pm \sqrt{q}) \in \operatorname{Crit}(W) \subset \mathcal{T}^{\vee}(\Lambda)$. Under the map (7) these map to $( \pm \sqrt{q}, \pm \sqrt{q}, \pm \sqrt{q}, \pm \sqrt{q}) \in \tilde{G}^{\vee}$. All of these solutions approach $y=0$ as $q \rightarrow 0$. 
(b) (Critical locus for a projective line with extra term) Continuing the example of the projective line $X / / G$ with potential with extra term from Example 1.11 (b) $W_{X, G}(y)=q^{3} / y^{2}+y+q / y$. The critical points are $y \sim \pm q^{1 / 2}$ and $y \sim-2 q^{2}$. Under the injection (7) these map to

$$
y(q) \sim\left(q^{2}, \pm q^{1 / 2}, \pm q^{1 / 2}\right)
$$

which converges to 0 as $q \rightarrow 0$; and

$$
y(q) \sim\left(q^{-1} / 4,-2 q^{-2},-q^{-1} / 2\right)
$$

which does not converge to 0 as $q \rightarrow 0$.

(c) (Critical locus for the stacky half-point) Continuing Example 1.11 (c), let $X / / G \cong$ $\mathbb{P}(2)$ so that

$$
W_{X, G}: \tilde{T} \cong \mathbb{Z}_{2} \rightarrow\{ \pm 1\} \subset \Lambda
$$

is the potential for the half-point. Then $\operatorname{Crit}\left(W_{X, G}\right)=\tilde{T}$ and $\operatorname{Jac}\left(W_{X, G}\right)$ is the group ring on $\mathbb{Z}_{2}$, isomorphic to the orbifold cohomology of $\mathbb{P}(2)=B \mathbb{Z}_{2}$.

An interpretation in terms of critical points that lie over the interior of the moment polytope is given in Proposition 3.19. We will prove the following identification with the Jacobian ring:

Theorem 1.15. For any rational symplectic class $\omega \in H_{2}^{G}(X)$, there is a canonical isomorphism

$$
T_{\kappa_{X}^{G}(\omega)} Q H(X / / G) \rightarrow \mathrm{Jac}_{+}\left(W_{X, G}\right) .
$$

Remark 1.16. (a) The left-hand-side $T_{\kappa_{X}^{G}(\omega)} Q H(X / / G)$ of (9) is independent of the presentation of $X / / G$ as a git quotient of $X$ by $G$. On the other hand, the righthand-side $\mathrm{Jac}_{+}\left(W_{X, G}\right)$ depends on the presentation.

(b) That the rings $T_{\kappa_{X}^{G}(\omega)} Q H(X / / G), \mathrm{Jac}_{+}\left(W_{X, G}\right)$ have the same dimension follows in the Fano case $c_{1}(X / / G)>0$ from Kouchnirenko's theorem [39, 3] Theorem 3.9 below. In general, we deduce the dimension equality

$$
\operatorname{dim}\left(T_{\kappa_{X}^{G}(\omega)} Q H(X / / G)\right)=\operatorname{dim}\left(\mathrm{Jac}_{+}\left(W_{X, G}\right)\right)
$$

from the toric minimal model program and an induction in Theorem 4.19 below. A similar procedure is used by Kawamata [37] to show the existence of an exceptional collection in the derived category $D^{b} \operatorname{Coh}(X / / G)$ of any toric orbifold $X / / G$.

(c) The Frobenius manifold structure $Q H(Y)$, including the pairing, is expected to be equivalent to Saito's Frobenius structure corresponding to the Landau-Ginzburg potential $W$, see for example Fukaya et al [21]. However, we do not discuss the Frobenius inner product in this paper.

We end the introduction with examples of the projective plane, written in different ways as a quotient:

Example 1.17. $\quad$ (a) (Projective plane as a quotient by a circle action) Suppose that $G=\mathbb{C}^{\times}$acts on $X=\mathbb{C}^{3}$ by scalar multiplication. Suppose that the polarization corresponds to a trivial line bundle with a negative weight on the fiber at the origin. 
The semistable locus is $X^{\mathrm{ss}}=X-\{0\}$ and the git quotient is $X / / G=\mathbb{P}^{2}$. We take the residual action of $T=\left(\mathbb{C}^{\times}\right)^{3} / \mathbb{C}^{\times}$to have moment polytope in $\mathfrak{t}^{\vee} \cong \mathbb{R}^{2}$ equal to

$$
\Delta_{X / / G}=\left\{\left(\lambda_{1}, \lambda_{2}\right) \in \mathbb{R}^{2} \mid \lambda_{1} \geq 0, \lambda_{2} \geq 0, \lambda_{1}+\lambda_{2} \leq 1\right\} .
$$

The corresponding potential is

$$
W_{X, G}\left(y_{1}, y_{2}\right)=y_{1}+y_{2}+q / y_{1} y_{2} .
$$

The critical points are the solutions to

$$
\begin{aligned}
& y_{1} \partial_{y_{1}} W_{X, G}\left(y_{1}, y_{2}\right)=y_{1}-q / y_{1} y_{2}=0 \\
& y_{2} \partial_{y_{2}} W_{X, G}\left(y_{1}, y_{2}\right)=y_{2}-q / y_{1} y_{2}=0
\end{aligned}
$$

Solutions are $y_{1}=y_{2}, \quad y_{1}^{3}=y_{2}^{3}=q$. These generators and relations give a presentation of the quantum cohomology of $\mathbb{P}^{2}$.

(b) (Projective plane as a quotient by a two-torus action) The projective plane $\mathbb{P}^{2}$ can be realized as a git quotient by a two-dimensional torus as follows. Suppose that $G=\left(\mathbb{C}^{\times}\right)^{2}$ acts on $X=\mathbb{C}^{4}$ with weights $(-1,1),(0,1),(1,1),(1,0)$. The symplectic quotient $X / / G$ is the "symplectic cut" of $\mathbb{C}^{2}$ by the circle actions with directions $(-1,1),(1,1)$ in the sense of Lerman [40]. The polytope $\Delta_{X / / G}$ is the intersection of a quadrant with two half-spaces with directions $(-1,1),(1,1)$ :

$$
\Delta_{X / / G}=\left\{\left(\lambda_{1}, \lambda_{2}\right) \in \mathbb{R}_{\geq 0}^{2} \mid \lambda_{1}+\lambda_{2} \leq c_{1},-\lambda_{1}+\lambda_{2} \leq c_{2}\right\}
$$

for some constants $c_{1}<c_{2}$. Suppose that the polarization corresponds to the weight $(2,1)$; this is the right-most chamber in Figure 1.

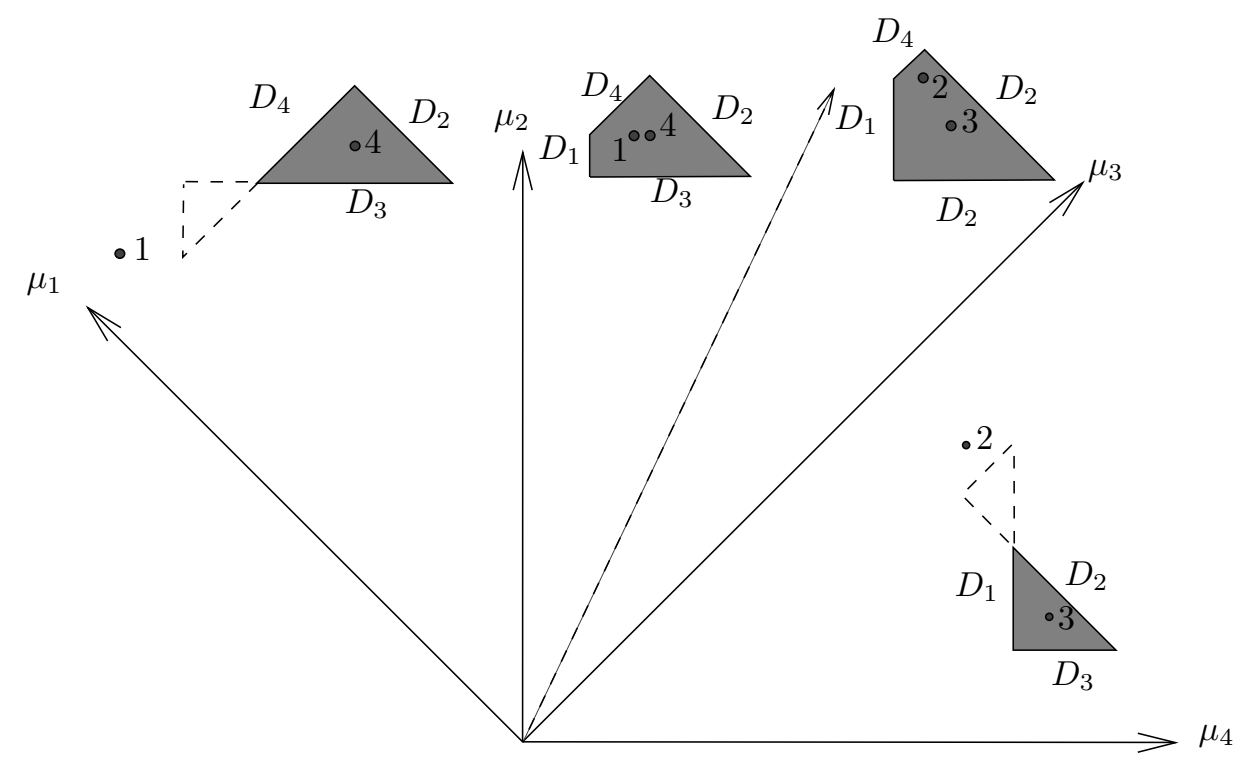

Figure 1. Chamber structure for git quotients and the moment images of the critical values, with multiplicities 
The semistable locus is

$$
X^{\mathrm{ss}}=\left\{x=\left(x_{1}, x_{2}, x_{3}, x_{4}\right) \mid x_{4} \neq 0,\left(x_{1}, x_{2}, x_{3}\right) \neq 0\right\} .
$$

The git quotient is $X / / G=\mathbb{P}^{2}$. In particular, the equation $x_{4}=0$ does not define a divisor in $X / / G$. The potential is

$$
W_{X, G}\left(y_{1}, y_{2}\right)=y_{1}+y_{2}+q / y_{1} y_{2}+q^{2} y_{1} / y_{2} \text {. }
$$

The partial derivatives are

$$
\begin{aligned}
& \partial_{y_{1}} W_{X, G}\left(y_{1}, y_{2}\right)=1-q / y_{1}^{2} y_{2}+q^{2} / y_{2}, \\
& \partial_{y_{2}} W_{X, G}\left(y_{1}, y_{2}\right)=1-q / y_{1} y_{2}^{2}-q^{2} y_{1} / y_{2}^{2} .
\end{aligned}
$$

The critical points, to leading order, are

$$
y_{1} \sim y_{2} \sim \exp (2 \pi i k / 3) q^{1 / 3}, k=0,1,2
$$

and the two critical points

$$
y_{1} \sim \pm i q^{-1 / 2}, \quad y_{2} \sim-2 q^{2}
$$

as shown in Figure 1. The first three (resp. second two) points (resp. do not) define elements of $\mathrm{Crit}_{+}\left(W_{X, G}\right)$. Hence $\mathrm{Crit}_{+}\left(W_{X, G}\right)$ consists of three reduced points, $Q H(X / / G) \cong \mathbb{C}^{\oplus 3}$. The other pictures in Figure 1 show the quotients for the other polarizations; the dotted line represents the ray $\mathbb{R}_{\geq 0} c_{1}^{G}(T X)$ generated by the equivariant first Chern class $c_{1}^{G}(T X)$, for which the quotient $X / / G$ has a potential with all critical points located at $0 \in \mathfrak{t}^{\vee}$. The number of critical points $y \in \operatorname{Crit}\left(W_{X, G}\right)$ mapping to each point in $\Psi(y) \in \Delta_{X / / G}$ is indicated in Figure 1. This ends the example.

We thank D. Cox, H. Iritani, D. McDuff, and C. Teleman for helpful comments.

\section{QUANTUM KIRWAN SURJECTIVITY FOR TORIC ORBIFOLDS}

In this section we prove surjectivity for the linearization of the quantum Kirwan map on a formal completion of equivariant quantum cohomology; the surjectivity also holds for the uncompleted cohomology but does not lead to an isomorphism. Let $X$ be a smooth polarized projective $G$-variety, or more generally, a smooth polarized quasiprojective $G$ variety convex at infinity in the sense of $[50,51,52]$, such as a finite-dimensional vector space with the action of a torus whose weights are contained in a half-space. The version of quantum Kirwan surjectivity we need involves a formal completion of the equivariant quantum cohomology. In this completion not only the powers of $q$ but also the degrees of the cohomology classes can go to infinity:

Definition 2.1. (Formal equivariant quantum cohomology ring) Let $\widehat{Q H}_{G}(X)$ be the vector space of infinite sums

$$
\widehat{Q H}_{G}(X)=\left\{\sum_{i=1}^{\infty} q^{\rho_{i}} \alpha_{i} \mid \alpha_{i} \in H_{G}^{a_{i}}(X), \inf _{i} \rho_{i}>-\infty, \lim _{i \rightarrow \infty} \rho_{i}+a_{i}=\infty\right\} .
$$


Equivalently, $\widehat{Q H}_{G}(X)$ is obtained by completing $H_{G}(X) \otimes \Lambda_{0}$ with respect to the degree filtration on $H_{G}(X)$, and then inverting $q$.

Remark 2.2. (Other completions) Note that there are various other natural completions. For example, completing $H_{G}(X) \otimes \Lambda$ with respect to the degree filtration on $H_{G}(X)$ gives a space of formal sums whose $q$-degree is not necessarily bounded below. The quantum Kirwan map does not extend to this formal completion. In the toric case the relationship between various completions is discussed by Fukaya et al in [21, Section 12].

Proposition 2.3. (Extension of the quantum Kirwan map to the formal equivariant quantum cohomology) Each Taylor coefficient $\kappa_{X}^{G, n}: Q H_{G}(X)^{n} \rightarrow Q H(X / / G), n \in \mathbb{Z}_{\geq 0}$ extends to a map $\widehat{Q H}_{G}(X)^{n} \rightarrow Q H(X / / G)$, still denoted $\kappa_{X}^{G, n}$.

Proof. The statement of the proposition follows from the properness result for scaled gauged maps with bounded energy: for any $e>0$, the set of non-empty $\overline{\mathcal{M}}_{n, 1}^{G}(\mathbb{A}, X, d)$ for which $\langle d, \omega\rangle<e$ is finite [27, Theorem 1.1]. In particular, for any energy bound $e$, the virtual dimensions of the components $\overline{\mathcal{M}}_{n, 1}^{G}(\mathbb{A}, X, d)$ of energy $\langle d, \omega\rangle<e$ are bounded from above by some number $f(e)$. Thus if $\alpha_{i} \in H_{G}^{a_{i}}(X), i=1, \ldots, n$ satisfy

$$
\sum_{i=1}^{n} a_{i}>f(e)+\operatorname{dim}(X / / G)
$$

then the push-forward of $\operatorname{ev}^{*}\left(\alpha_{1}, \ldots, \alpha_{n}\right) \in H\left(\overline{\mathcal{M}}_{n, 1}^{G}(\mathbb{A}, X, d)\right)$ to $\bar{I}_{X / / G}$ is zero for reasons of dimension. If $\alpha \in H_{G}(X)$ has degree bounded from below by $f(e)+\operatorname{dim}(X / / G)$ then the contribution of $\kappa_{X}^{G, n}(\alpha)$ contains only terms with $q$-degree at least $e$. The claim follows.

We now partially compute the quantum Kirwan map in the toric case. Let $X$ be a finite-dimensional complex vector space with an action of a complex torus $G$, with weights $\mu_{1}, \ldots, \mu_{k}$ contained in an open half-space and equipped with a polarization so that the quotient $X / / G$ is locally free. Our first step is to classify the affine gauged maps which appear in the definition of $\kappa_{X}^{G}$ :

Theorem 2.4. (Classification of affine gauged maps in the toric case) An affine gauged map to $X / G$ of homology class $d \in H_{2}^{G}(X, \mathbb{Q})$ is equivalent to a morphism $u=\left(u_{1}, \ldots, u_{k}\right)$ : $\mathbb{A} \rightarrow X$ satisfying the conditions that

(a) the degree of $u_{j}$ is at most $\left\langle\mu_{j}, d\right\rangle$; and

(b) if $u(\infty)=\left(u_{j}(\infty)=\left\{\begin{array}{ll}u_{j}^{\left\langle\mu_{j}, d\right\rangle} /\left\langle\mu_{j}, d\right\rangle ! & \left\langle\mu_{j}, d\right\rangle \in \mathbb{Z}_{\geq 0} \\ 0 & \text { otherwise }\end{array}\right)_{j=1}^{k}\right.$ denotes the vector of leading order coefficients with integer exponents, then $u(\infty) \in X^{\mathrm{ss}}$.

Thus $\mathcal{M}_{1,1}^{G}(\mathbb{A}, X, d)^{G}$ resp. $\mathcal{M}_{1,0}^{G}(\mathbb{A}, X, d)^{G}$ is the quotient of the space of such morphisms by the action of $G$ resp. the action of $G$ and translation.

Example 2.5. (Examples of the classification of affine gauged maps) 
(a) (Jaffe-Taubes classification) If $G=\mathbb{C}^{\times}$acts on $X=\mathbb{C}$ with weight 1 then $\mathcal{M}_{1,1}^{G}(\mathbb{A}, X, d)$ consists of polynomials $u(z)$ of degree exactly $d$ quotiented by the action of $G$. Since any such polynomial $u(z)$ is classified by its roots $z, u(z)=0$, we have $\mathcal{M}_{1,1}^{G}(\mathbb{A}, X, d) \cong \operatorname{Sym}^{d}(\mathbb{A})$.

(b) (Stacky half-point) Continuing Examples 1.11 (c) and 1.14 (c), suppose $G=\mathbb{C}^{\times}$ acts on $X=\mathbb{C}$ with weight 2 . Let $d$ to be a half-integer. The moduli stack $\mathcal{M}_{1,1}^{G}(\mathbb{A}, X, d)$ consists of non-zero polynomials $u(z)$ of degree $2 d$ quotiented by the action of $G$. Since any such polynomial is classified by its roots, $\overline{\mathcal{M}}_{1,1}^{G}(\mathbb{A}, X, d) \cong$ $\operatorname{Sym}^{2 d}(\mathbb{A})$. The evaluation map to $X / / G=\mathbb{P}(2)$ maps to the trivial resp. twisted sector if $2 d$ is even resp. odd.

(c) (Projective space) If $G=\mathbb{C}^{\times}$acts on $X=\mathbb{C}^{k}$ by scalar multiplication then $\mathcal{M}_{1,1}^{G}(\mathbb{A}, X, d)$ consists of tuples $\left(u_{1}, \ldots, u_{k}\right)$ of polynomials of degree most $d$ such that at least one of the polynomials $u_{j}$ is degree exactly $d$, quotiented by the action of $G$. One sees that $\mathcal{M}_{1,1}^{G}(\mathbb{A}, X, d)$ is a vector bundle over $\mathbb{P}^{k-1}$ of rank $d k$.

Proof of Theorem. By definition, a morphism $u: \mathbb{P}(1, r) \rightarrow X / G$ consists of a $G$-bundle $P \rightarrow \mathbb{P}(1, r)$ and a section of the associated $X$-bundle $u: \mathbb{P}(1, r) \rightarrow P \times_{G} X$. The bundle can be described by a clutching function $z \mapsto z^{\lambda / r}$ for some $\lambda \in \mathfrak{g}_{\mathbb{Z}}^{\vee}$ with $\lambda / r=d$. The first condition (a) is the condition that a map $\mathbb{A} \rightarrow X$ extend to a global section. The second condition (b) is that the extension maps $\mathbb{P}(r)$ to the semistable locus $X / / G$. The representability condition for the morphism $u$ is that the image of $\mathbb{P}(r)$ is a point $u(\infty)$ in $X / / G$ with automorphism group containing a group $\mathbb{Z}_{r}$ generated by $\exp (\lambda / r)$, so that $\lambda / r$ is the minimal representation of $d$.

Theorem 2.6. (Quantum Kirwan surjectivity, toric case) For any rational symplectic class $\omega \in H_{2}^{G}(X, \mathbb{Q})$, the map $D_{\omega} \kappa_{X}^{G}: T_{\omega} \widehat{Q H}_{G}(X) \rightarrow T_{\kappa_{X}^{G}(\omega)} Q H(X / / G)$ is surjective.

Example 2.7. (The case of a free quotient) If $X / / G$ is a smooth variety, that is, has no orbifold points, then the statement of Theorem 2.6 follows from Kirwan's surjectivity result from [38], or an explicit description of the classical Kirwan map in the toric case. Indeed the leading order term (setting the Novikov parameter $q$ to zero) is the classical Kirwan map. The novelty of the above theorem is that in case that $X / / G$ is an orbifold, the twisted sectors in $Q H(X / / G)$ are also in the image of the quantum Kirwan map, so that $T_{\kappa_{X}^{G}(\omega)} Q H(X / / G)$ is a quotient of the usual ring of polynomial invariants $T_{\omega} Q H_{G}(X) \cong$ $\operatorname{Sym}\left(\mathfrak{g}^{\vee}\right)^{G} \otimes \Lambda$.

The proof of Theorem 2.6 in general relies on the following computation which we call a fractional Batyrev relation based on similarity with Batyrev [4].

Notation 2.8. (a) (Ceiling) The ceiling $\lceil x\rceil$ is the smallest integer greater than or equal to $x$.

(b) (Classification of twisted sectors) We identify $H_{2}^{G}(X) \cong \mathfrak{g}$ and $H_{G}^{2}(X) \cong \mathfrak{g}^{\vee}$. Any $d \in H_{2}^{G}(X)$ thus defines an element $\exp (d) \in G$. This element corresponds to a summand in $H\left(I_{X / / G}\right)$ if it has non-trivial fixed point set in $X^{\text {ss }}$. 
(c) (Identity in each twisted sector) For such $d$ we denote by $1_{\exp (d)} \in H\left(I_{X / / G}\right)$ the degree zero class in the twisted sector (which will have non-zero degree with respect to the grading on $Q H(X / / G)$.)

(d) (Divisor classes in twisted sectors) For each $j=1, \ldots, k$, we denote by $\nu_{j} 1_{\exp (d)}$ the (possibly empty) divisor class in the twisted sector for $\exp (d)$, obtained by setting the $j$-th coordinate $x_{j}$ equal to 0 . The product of the classes $\nu_{j}$ with $1_{\exp (d)}$ is the product of these cohomology classes in the twisted sector in $H\left(I_{X / / G}\right)$.

Proposition 2.9 (Fractional Batyrev relation). For any $d \in \mathfrak{g}$ such that $\exp (d)$ has nonempty fixed point set in $X^{\mathrm{ss}}$ and $\mathcal{M}_{1,1}(\mathbb{A}, X, d)$ is non-empty,

$$
D_{\omega} \kappa_{X}^{G}\left(\prod_{\left\langle\mu_{j}, d\right\rangle \geq 0} \mu_{j}^{\left\lceil\left\langle\mu_{j}, d\right\rangle\right\rceil}\right)=\prod_{\left\langle\mu_{j}, d\right\rangle \leq 0} \nu_{j}^{-\left\lceil\left\langle\mu_{j}, d\right\rangle\right\rceil} q^{\langle\omega, d\rangle} 1_{\exp (d)}+\text { higher order in } q .
$$

Proof of Proposition 2.9. Recall that $\overline{\mathcal{M}}_{1,1}^{G}(\mathbb{A}, X, d)$ is the stack of once-marked stable scaled affine gauged maps to $X[50,51,52]$. In general, this compactification allows bubbles in $X / / G$ and ghost bubbles in $X$ when the markings $z_{1}, \ldots, z_{n}$ on the domain come together. However, since $X$ is affine, there are no non-constant holomorphic maps $u: \mathbb{P}^{1} \rightarrow X$ from projective lines $\mathbb{P}^{1}$ to $X$. Thus any element of $\overline{\mathcal{M}}_{1,1}^{G}(\mathbb{A}, X, d)$ consists of components that are affine gauged maps to $X$, and components that are stable maps to $X / / G$. We wish to compute the virtual push-forward under ev $\infty: \overline{\mathcal{M}}_{1,1}^{G}(\mathbb{A}, X, d) \rightarrow \bar{I}_{X / / G}$ of

$$
\mathrm{ev}^{*} \prod_{\left\langle\mu_{j}, d\right\rangle \geq 0} \mu_{j}^{\left\lceil\left\langle\mu_{j}, d\right\rangle\right\rceil}=\mathrm{ev}^{*} \operatorname{Eul}\left(\prod_{\left\langle\mu_{j}, d\right\rangle \geq 0} \mathbb{C}_{\mu_{j}}^{\left\lceil\left\langle\mu_{j}, d\right\rangle\right\rceil}\right) .
$$

On the families of affine gauged maps considered here, there is a lift of $\mathrm{ev}_{\infty}$ from the rigidified inertia stack $\bar{I}_{X / / G}$ to $I_{X / / G}$, and we may ignore the rigidification. Consider the section

$$
\sigma: \overline{\mathcal{M}}_{1,1}^{G}(\mathbb{A}, X, d) \rightarrow \mathrm{ev}^{*} \prod_{\left\langle\mu_{j}, d\right\rangle \geq 0} \mathbb{C}_{\mu_{j}}^{\left\lceil\left\langle\mu_{j}, d\right\rangle\right\rceil}, \quad u \mapsto\left(u_{j}^{(i)}\left(z_{1}\right)\right)_{j=1, i=0}^{k,\left\lceil\left\langle\mu_{j}, d\right\rangle\right\rceil}
$$

consisting of the derivatives $u^{(i)}$ of $u$ at the marking $z_{1}$; note this is well-defined because of the scaling on the domain, that is, we are modding out by translation on the domain only. On the stratum $\mathcal{M}_{1,1}^{G}(\mathbb{A}, X), \sigma$ has zeroes corresponding to maps with all lower-order terms vanishing. The restriction of $\mathrm{ev}_{\infty}$ to $\sigma^{-1}(0)$ defines an isomorphism

$$
\left.\mathrm{ev}_{\infty}\right|_{\sigma^{-1}(0)}: \sigma^{-1}(0) \rightarrow \bar{I}_{X / / G}(\exp (d)) \cap\left\{x_{j}=0,\left\langle\mu_{j}, d\right\rangle \leq-1\right\}
$$

where $\bar{I}_{X / / G}(\exp (d))$ is the sector with stabilizer $\exp (d)$, defined as the git quotient of the subspace of $X$ corresponding to coordinates $x_{j}$ with $\left\langle d, \mu_{j}\right\rangle \in \mathbb{Z}$. The obstruction space at any morphism $u$ is the higher cohomology of the vector bundle $P \times_{G} X$. This higher cohomology may be identified via duality with the span of monomials whose $j$-th component has degree strictly between 0 and $-\left\lceil\left\langle\mu_{j}, d\right\rangle\right\rceil$. Thus the obstruction bundle has 
Euler class

$$
\operatorname{Eul}\left(\prod_{\left\langle\mu_{j}, d\right\rangle \leq-1} \mathbb{C}_{\mu_{j}}^{-\left\lceil\left\langle\mu_{j}, d\right\rangle\right\rceil-1}\right) .
$$

It follows that the contribution to the pushforward (10) from the stratum with irreducible domain is

as claimed.

$$
\left(\prod_{\left\langle\mu_{j}, d\right\rangle \leq-1} \nu_{j}^{-\left\lceil\left\langle\mu_{j}, d\right\rangle\right\rceil-1+1}\right) 1_{\exp (d)}
$$

Next we examine contributions from the boundary. Any boundary configuration in $\overline{\mathcal{M}}_{\Gamma}(\mathbb{A}, X, d)-\mathcal{M}_{\Gamma}(\mathbb{A}, X, d)$ contains a component with a marking and a gauged map $u: \mathbb{A} \rightarrow X$ of class $d^{\prime}$ with $\left\langle d^{\prime}, \omega\right\rangle<\langle d, \omega\rangle$ together with other components that are morphisms to $X / / G$ and affine gauged maps without markings. The zero set $\sigma^{-1}(0)$ of $\sigma$ on such a stratum $\mathcal{M}_{\Gamma}(\mathbb{A}, X)$ consists of configurations where $u: C \rightarrow X / G$ has $j$-th component $u_{j}=0$ if $\left\langle\mu_{j}, d\right\rangle>\left\langle\mu_{j}, d^{\prime}\right\rangle \in \mathbb{Z}$. So the components $u_{j}$ with non-zero leading order correspond to $j$ with $\left\langle\mu_{j}, d\right\rangle \leq\left\langle\mu_{j}, d^{\prime}\right\rangle$. Clearly, the convex hull hull $\left(\left\{\mu_{j}\right\}\right)$ of such weights cannot contain $\omega$, since $\left\langle d-d^{\prime}, \omega\right\rangle>0$ but $\left\langle d-d^{\prime}, \mu_{j}\right\rangle \leq 0$. By the description of the unstable locus in (4), the asymptotic limit of points in $\sigma^{-1}(0)$ consist of unstable points in $X$. So the zero set $\sigma^{-1}(0)$ is empty.

Finally we consider the integral

$$
\int_{\left[\overline{\mathcal{M}}_{1,1}^{G}\left(\mathbb{A}, X, d^{\prime}\right)\right]} \operatorname{ev}^{*} \prod_{\left\langle\mu_{j}, d\right\rangle \geq 0} \mu_{j}^{\left\lceil\left\langle\mu_{j}, d\right\rangle\right\rceil}
$$

for $d \neq d^{\prime}$. The same argument as in the previous paragraph shows that the integral (11) is zero unless $\langle d, \omega\rangle \leq\left\langle d^{\prime}, \omega\right\rangle$. But since $\omega$ is generic, this inequality implies strict inequality. Hence the contributions (11) from such degrees $d^{\prime}$ are of higher energy than the leader order terms.

Corollary 2.10. (Surjectivity onto twisted units) For any $g \in G$ with non-trivial stabilizer in $X^{\mathrm{ss}}$, there exists an element $d \in \mathfrak{g}$ with $\exp (d)=g$ and $\left\langle d, \mu_{j}\right\rangle>0$ for all $j=1, \ldots, k$ and thus

$$
D_{\omega} \kappa_{X}^{G}\left(\prod_{j=1}^{k} \mu_{j}^{\left\lceil\left\langle\mu_{j}, d\right\rangle\right\rceil}\right)=1_{\exp (d)} q^{\langle d, \omega\rangle} \quad \bmod \text { higher order in } q \text {. }
$$

Proof. Since the weights $\mu_{j}, j=1, \ldots, k$ are contained in a half-space, there exists a vector $\zeta \in \mathfrak{g}_{\mathbb{R}}$ such that $\left\langle\zeta, \mu_{j}\right\rangle>0$ for $j=1, \ldots, k$. Let $U \subset \mathfrak{g}$ be a compact subset such that $\exp (U)=G$. Then $c \zeta+U$ contains the desired vector $d$, for $c \gg 0$.

Notation 2.11. (Cohomology classes in twisted sectors) For any $j \in\{1, \ldots, k\}$ such that $\left\langle\mu_{j}, d\right\rangle \in \mathbb{Z}$ denote by $1_{\exp (d)} \delta_{j} \in H^{2}\left(I_{X / / G}\right)$ the corresponding divisor class in the twisted sector corresponding to $\exp (d)$. Let

$$
1_{\exp (d)} \delta_{J}=1_{\exp (d)} \prod_{j \in J} \delta_{j} \in H\left(I_{X / / G}\right)
$$


be the classical product of divisor classes in the twisted sector for $\exp (d)$. Since each component of $I_{X / / G}$ is itself a rationally smooth toric stack, any cohomology class of $I_{X / / G}$ arises in this way by the classical description by Danilov-Jurkiewicz [16, 35, 36].

Corollary 2.12. (Surjectivity onto twisted sectors) With $d$ as in Corollary 2.10, for any subset $J$ of $\left\{j,\left\langle\mu_{j}, d\right\rangle \in \mathbb{Z}\right\}$,

$$
D_{\omega} \kappa_{X}^{G}\left(\prod_{j=1}^{k} \mu_{j}^{\left\lceil\left\langle\mu_{j}, d\right\rangle\right\rceil} \prod_{j \in J} \mu_{j}\right)=1_{\exp (d)} \delta_{J} q^{\langle d, \omega\rangle} \quad \bmod \text { higher order in } q .
$$

Proof of Theorem 2.6. For each $(g, J)$ let $\alpha_{g, J}:=1_{g} \delta_{J} \in H\left(I_{X / / G}\right)$ and choose an element $\tilde{\alpha}_{g, J}$ such that $D_{\omega} \kappa_{X}^{G}\left(\tilde{\alpha}_{g, J}\right)$ is equal to $\alpha_{g, J}$ plus terms of higher energy. A recursion produces an inverse. (Note that by choosing a basis, one obtains an inverse involving a finite number of classes in $H_{G}(X)$, that is, the formal completion is not necessary for surjectivity.)

We give several examples.

Example 2.13. (a) (Stacky half-point) Continuing Example 2.5 (b), let $X=\mathbb{C}$ with $G=\mathbb{C}^{\times}$acting with weight 2 , so that $X / / G=\mathbb{P}(2)=B \mathbb{Z}_{2}$. The quantum cohomology is $Q H(X / / G)=\Lambda \oplus \Lambda$, corresponding to the stabilizers 1, -1 , called the untwisted and twisted sectors. We identify $\mathfrak{g}_{\mathbb{Z}}^{\vee}=\mathbb{Z}$ in the standard way. By the classification Theorem 2.4 the class zero component consists of constant maps

$$
\overline{\mathcal{M}}_{1,1}^{G}(\mathbb{A}, X, 0)=(X-\{0\}) / G \cong \mathbb{P}(2) .
$$

The class-1/2 component is of dimension one:

$$
\mathcal{M}_{1,1}^{G}(\mathbb{A}, X, 1 / 2)=\left\{c_{1} z+c_{0}, c_{1} \neq 0\right\} / G \cong \mathbb{C} / \mathbb{Z}_{2} .
$$

If $Q H_{G}(X)=\Lambda[\xi]$ with generator the Euler class $\xi$ of the weight one representation then the fractional Batyrev relations give

$$
D_{\omega} \kappa_{X}^{G}(1)=1, \quad D_{\omega} \kappa_{X}^{G}(\xi)=\left(q^{1 / 2} / 2\right) \theta_{-}, \quad D_{\omega} \kappa_{X}^{G}\left(\xi^{2}\right)=q / 4 .
$$

Thus $D_{\omega} \kappa_{X}^{G}$ is surjective and we obtain a presentation

$$
T_{\kappa_{X}^{G}(\omega)} Q H(\mathbb{P}(2)) \cong \Lambda[\xi] /\left(\xi^{2}-q / 4\right) .
$$

Because $c_{1}^{G}(X)$ is positive on curve classes, in this case $\kappa_{X}^{G}(0)=0$ and $\kappa_{X}^{G}(\omega)$ is the reduced symplectic class. Note that under the identification $\xi \mapsto q^{1 / 2} / 2 \theta_{-}$this agrees with the isomorphism of $Q H(\mathbb{P}(2))$ with the group ring of $\mathbb{Z}_{2}$ in Example 1.2 .

(b) (Teardrop orbifold) If $G=\mathbb{C}^{\times}$acts on $X=\mathbb{C}^{2}$ with weights 1,2 , so that $X / / G=$ $\mathbb{P}(1,2)$ is the teardrop orbifold,

$$
D_{\omega} \kappa_{X}^{G}(\xi)=\theta_{+}, \quad D_{\omega} \kappa_{X}^{G}\left(\xi^{2}\right)=q^{1 / 2} \theta_{-} / 2, \quad D_{\omega} \kappa_{X}^{G}(\xi)^{3}=q / 4 .
$$

Thus $D_{0} \kappa_{X}^{G}$ is surjective and we obtain a presentation

$$
T_{\kappa_{X}^{G}(\omega)} Q H(\mathbb{P}(1,2))=\Lambda[\xi] /\left(\xi^{3}-q / 4\right) .
$$


See Coates-Lee-Corti-Tseng [11] and Mann [41] for more on the quantum cohomology of weighted projective spaces.

Remark 2.14. (Generation by divisor classes) In particular, our version of quantum Kirwan surjectivity implies that $Q H(X / / G)$ is generated by the "divisor classes" $D_{\omega} \kappa_{X}^{G}\left(\mu_{i}\right)$. Note that this is the case even if $X / / G$ has no divisors, for example, when $X / / G$ is the stacky half-point $\mathbb{P}(2)$ then $Q H(X / / G) \cong \Lambda\left[\mathbb{Z}_{2}\right]$ is the group ring of $\mathbb{Z}_{2}$. These "divisor classes" are degree two only when working over the larger Novikov ring $\Lambda_{X}^{G}$ to achieve a $\mathbb{Z}$-grading.

\section{Quantum Stanley-Reisner Ring And Jacobian Ring}

In this section we identify the quantum Stanley-Reisner ring with the Jacobian ring, and discuss various extensions to formal versions. Consider a git quotient $X / / G$ of a finitedimensional complex vector space $X$ by the action of a complex torus $G$ with weights $\mu_{1}, \ldots, \mu_{k}$ contained in an open half-space and spanning $\mathfrak{g}^{\vee}$.

Definition 3.1. (a) (Classical Stanley-Reisner ideal) The Stanley-Reisner ideal $S R_{X}^{G}$ in $Q H_{G}(X)$ is the ideal generated by products of weights

$$
\mu_{I}=\prod_{i \in I} \mu_{i} \in Q H_{G}^{2|I|}(X)
$$

where $I$ is a primitive collection $I \subset\{1, \ldots, k\}$ with respect to the fan of $X / / G$ : the set $X_{I}=\left\{x_{i}=0, i \in I\right\}$ is contained in the unstable locus of $X$ and $I$ is a minimal subset with this property.

(b) (Classical Stanley-Reisner ring) The Stanley-Reisner ring is the quotient of $H_{G}(X)$ by the Stanley-Reisner ideal $S R_{X}^{G}$.

Theorem 3.2. (Rational cohomology of a projective simplicial toric variety) Suppose that stable $=$ semistable for the $G$-action on $X$. Then the Kirwan map $H_{G}(X, \mathbb{Q}) \rightarrow H(X / / G, \mathbb{Q})$ induces an isomorphism $H_{G}(X, \mathbb{Q}) / S R_{X}^{G} \rightarrow H(X / / G, \mathbb{Q})$.

Proof. This is essentially the Danilov-Jurkiewicz description of the cohomology ring [16, $35,36]$, using the fact that each weight function $\mu_{i} \in \mathfrak{g}_{\mathbb{Q}}^{\vee} \cong H_{G}^{2}(X, \mathbb{Q})$ maps to the corresponding divisor class in $H(X / / G, \mathbb{Q})$ under the classical Kirwan map. A description from the point of view of equivariant cohomology can be found in Bonavero-Brion [6].

Note that there are no "linear relations" in the above description; these are in the standard description the kernel of the map $H_{\tilde{G}}(X, \mathbb{Q}) \rightarrow H_{G}(X, \mathbb{Q})$, where $\tilde{G}$ is the "big torus" from Notation 1.9.

Definition 3.3. (Formal quantum Stanley-Reisner ring)

(a) The formal quantum Stanley-Reisner ideal $\widehat{Q S R}_{X}^{G} \subset \widehat{Q H}_{G}(X, \mathbb{Q})$ is the completion (or equivalently, the closure) of $Q S R_{X, G}$ in $\widehat{Q H}_{G}(X, \mathbb{Q})$. The quotient $\widehat{Q H}_{G}(X, \mathbb{Q}) / \widehat{Q S R}_{X}^{G}$ is the formal Batyrev ring. 
(b) The equivariant resp. formal equivariant quantum Stanley-Reisner ideal resp. Batyrev ring are obtained by replacing the expressions $\mu_{j}$ by their unrestricted versions. Denote by $\epsilon_{1}, \ldots, \epsilon_{k} \in \tilde{\mathfrak{g}}^{\vee} \cong H_{\tilde{G}}^{2}(X)$ the coordinates (weights) on the big torus $\tilde{G}$ acting on $X$ and so that $\mu_{1}, \ldots, \mu_{k} \in \mathfrak{g}^{\vee} \cong Q H_{G}^{2}(X)$ are their restrictions to $\mathfrak{g}$. The equivariant quantum Stanley-Reisner ideal is the closure of the ideal generated by

$$
Q S R_{X}^{G, \tilde{G}}(d):=\prod_{\left\langle\mu_{j}, d\right\rangle \geq 0} \epsilon_{j}^{\left\langle\mu_{j}, d\right\rangle}-q^{\langle d, \omega\rangle} \prod_{\left\langle\mu_{j}, d\right\rangle \leq 0} \epsilon_{j}^{-\left\langle\mu_{j}, d\right\rangle} .
$$

Example 3.4. (Batyrev relations for a quotient of affine four-space by a two-torus) Let $G=\left(\mathbb{C}^{\times}\right)^{2}$ acting on $X=\mathbb{C}^{4}$ with weights $(-1,1),(0,1),(1,1),(1,0)$. The corresponding chamber structure and polytopes of the quotients are shown in the Figure 1. The equivariant quantum Stanley-Reisner relations include

$$
\epsilon_{1}^{-d_{1}+d_{2}} \epsilon_{2}^{d_{2}} \epsilon_{3}^{d_{1}+d_{2}} \epsilon_{4}^{d_{1}}=q^{d_{1}+d_{2}}, \quad-d_{1}+d_{2}, d_{1} \geq 0
$$

where $\epsilon_{i}$ are the equivariant generators from Definition 3.3. In particular we have

$$
\epsilon_{1} \epsilon_{2} \epsilon_{3}=q, \quad \epsilon_{2} \epsilon_{3}^{2} \epsilon_{4}=q^{2} .
$$

Notice the first relation in (12) defines the quantum cohomology $Q H(X / / G)$ for the quotient $X / / G$ in the right-most chamber in Figure 1. The second relation in (12) defines quantum cohomology for the quantum cohomology in the left-most chamber. The nonequivariant relations are

$$
\left(-\xi_{1}+\xi_{2}\right)\left(\xi_{2}\right)\left(\xi_{1}+\xi_{2}\right)=q, \quad \xi_{2}\left(\xi_{1}+\xi_{2}\right)^{2} \xi_{1}=q^{2} .
$$

Proposition 3.5. (Inclusion of the Batyrev relations in the kernel of the quantum Kirwan map) $[50,51,52]$ The kernel of the linearized quantum Kirwan map

$$
D_{\alpha} \kappa_{X}^{G}: T_{\alpha} Q H_{G}(X) \rightarrow T_{\kappa_{X}^{G}(\alpha)} Q H(X / / G)
$$

contains the quantum Stanley-Reisner ideal for $\alpha$ in a formal neighborhood of the symplectic class $\omega \in Q H_{G}^{2}(X)$. So there is a quotient map

$$
T_{\alpha} Q H_{G}(X) / Q S R_{X, G}(\alpha) \rightarrow T_{\kappa_{X}^{G}(\alpha)} Q H(X / / G) .
$$

Proof. The adiabatic limit theorem of $[50,51,52]$ relates the localized genus zero graph potential $\tau_{X / / G,-} \in H(X / / G)\left[\left[\hbar^{-1}\right]\right]$ of $X / / G$ with the localized gauged graph potential $\tau_{X,-}^{G} \in H_{G}(X)\left[\left[\hbar^{-1}\right]\right]$ of $X$,

$$
\tau_{X,-}^{G}(1, \hbar, q)=\sum_{d \in H_{2}^{G}(X)} q^{d} \frac{\prod_{j=1}^{k} \prod_{m=-\infty}^{\left\langle\mu_{j}, d\right\rangle}\left(\mu_{j}+m \hbar\right)}{\prod_{j=1}^{k} \prod_{m=-\infty}^{0}\left(\mu_{j}+m \hbar\right)}
$$

The localized gauged graph potential $\tau_{X,-}^{G}(1, \hbar, q)$ is defined by virtual enumeration of quasimaps, or more generally, Mundet-stable maps to the quotient stack. The localized gauged potential is the solution to the Gelfand-Kapranov-Zelevinsky hypergeometric system, see for example Iritani [32], Cox-Katz [14, (11.92)]. The Batyrev relations correspond 
to differential operators of that system,

$$
\square_{d}=\prod_{\left\langle\mu_{j}, d\right\rangle \geq 0} \partial_{j}^{\left\langle\mu_{j}, d\right\rangle}-q^{\langle\omega, d\rangle} \prod_{\left\langle\mu_{j}, d\right\rangle \leq 0} \partial_{j}^{-\left\langle\mu_{j}, d\right\rangle}
$$

Now $\tau_{X / / G,-}$, as in Givental [23] is a fundamental solution for the quantum differential equation on $X / / G$. It follows that any differential operator that annihilates the localized gauged graph potential, is transformed via $D_{\omega} \kappa_{X}^{G}$ to a differential operator annihilating the fundamental solution, and so defines a relation.

The following is a direct, geometric argument using the fundamental property of the Euler class. Define bundles

$$
E_{ \pm}:=\bigoplus_{\left( \pm \mu_{j}(d) \geq 0\right.} \mathrm{ev}_{1}^{*} \mathbb{C}_{\mu_{j}}^{\oplus \mu_{j}(d)}
$$

The Euler class of $E_{ \pm}$is

$$
\zeta_{ \pm}(d):=\operatorname{Eul}\left(E_{ \pm}\right)=\prod_{ \pm \mu_{j}(d) \geq 0} \mu_{j}^{\mu_{j}(d)}
$$

Let $\sigma$ denote the section of $E_{+}$given by the taking the derivatives $\sigma_{i, j}, i=1, \ldots, d_{j}=$ $\min \left(\mu_{j}(d), \mu_{j}\left(d^{\prime}\right)\right)$ of $u$ at $z=z_{1}$. Consider the diagram

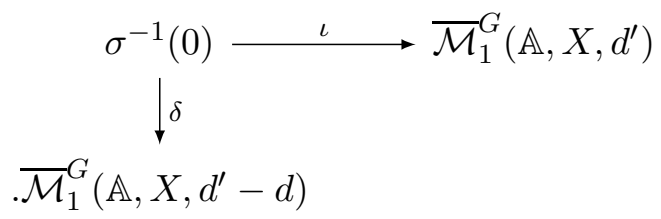

defined as follows. The map $\iota$ is the inclusion. To construct $\delta$, note that $\sigma^{-1}(0)$ consists of maps whose $j$-th component vanishes to order $d_{j}$ at the marking $z_{1}$. Therefore, for any $[u] \in \sigma^{-1}(0)$ we may define new map of degree $d^{\prime}-d$ by dividing by the $i$-th component of $u: C \rightarrow X / G$ on the component of $C$ containing $z_{1}$ by $\left(z-z_{1}\right)^{d_{i}}$ on the component $C_{1}$ containing $z_{1}$, and otherwise leaving the map unchanged, to obtain a map denoted $\left(z-z_{1}\right)^{-d} u$. Note that this is a change of $u$ by the action of an element of the oneparameter subgroup corresponding to $d$. Thus there is a canonical map

$$
\delta: \sigma^{-1}(0) \rightarrow \overline{\mathcal{M}}_{1}^{G}\left(\mathbb{A}, X, d^{\prime}-d\right), \quad u \mapsto\left[u /\left(z-z_{1}\right)^{d}\right] .
$$

Similarly the Euler class of the normal bundle to $\delta$ is, if non-empty, the product of classes $\mu_{j}^{\min \left(\mu_{j}(-d), \mu_{j}\left(d^{\prime}-d\right)\right)}$ for $\mu_{j}(d)<0$.

The remaining factors are accounted for by the difference in perfect obstruction theories. We denote by $p^{d^{\prime}}$ the restriction of the map $p$ from the universal curve to maps of homology class $d^{\prime}$. To compute the difference in classes we note that (if $e^{d^{\prime}}, e^{d^{\prime}-d}$ denote the universal 
evaluation maps)

$$
\begin{aligned}
\iota^{*}\left[R p_{*}^{d^{\prime}} e^{*} T(X / G)\right]-\delta^{*}\left[R p_{*}^{d^{\prime}-d} e^{*} T(X / G)\right]= & \iota^{*}\left[R p_{*}^{d^{\prime}} \sum_{j}\left(\mathcal{O}_{z_{1}}\left(\mu_{j}\left(d^{\prime}\right)\right)\right]\right. \\
& -\delta^{*}\left[R p_{*}^{d^{\prime}-d} \sum_{j} \mathcal{O}_{z_{1}}\left(\mu_{j}\left(d^{\prime}-d\right)\right)\right] \\
= & \iota^{*}\left[E_{+}\right]-\delta^{*}\left[E_{-}\right] .
\end{aligned}
$$

Hence for any class $\alpha_{0} \in H(X / / G)$ we obtain

$$
\int_{\overline{\mathcal{M}}_{1}^{G}\left(\mathbb{A}, X, d^{\prime}\right)} \mathrm{ev}_{0}^{*} \alpha_{0} \otimes \mathrm{ev}_{1}^{*} \zeta_{+}(d)=\int_{\overline{\mathcal{M}}_{1}^{G}\left(\mathbb{A}, X, d^{\prime}-d\right)} \mathrm{ev}_{0}^{*} \alpha_{0} \otimes \mathrm{ev}_{1}^{*} \zeta_{-}(d) .
$$

By definition of the quantum Kirwan map this implies

$$
D_{0} \kappa_{X}^{G}\left(\zeta_{+}(d)\right)=q^{d} D_{0} \kappa_{X}^{G}\left(\zeta_{-}(d)\right) .
$$

which proves the claim.

Similar results were obtained for toric manifolds under the name of mirror theorems in, for example, Iritani [34], by writing the toric variety as a complete intersection in another Fano toric variety and applying the Givental formalism, and for toric orbifolds in Coates, Corti, Iritani, and Tseng [12], [13, Theorem 5.13].

Example 3.6. (The second Hirzebruch surface, as in [26, Example 3.5]) The second Hirzebruch surface $F_{2}$ is a quotient of $X=\mathbb{C}^{4}$ by the $G=\mathbb{C}^{\times, 2}$-action with weights $(0,1),(-2,1),(1,0),(1,0)$. Let $p_{1}, p_{2} \in H_{2}(X / / G)$ be the the zero section and fiber classes. The divisor classes are

$$
D_{1}=p_{2}, \quad D_{2}=p_{2}-2 p_{1}, \quad D_{3}=D_{4}=p_{1} .
$$

In the case of nef toric varieties it follows from the adiabatic limit theorem of $[50,51,52]$ that the quantum Kirwan map intertwines the $I$-function and $J$-function of Givental [23], and so must agree with the mirror transformation for the second Hirzebruch surface, computed in Cox-Katz [14, Example 11.2.5.2]. Let $s_{1}, s_{2}$ resp $r_{1}, r_{2}$ be the coordinates on the torus with Lie algebra $H_{G}^{2}(X, \mathbb{C})$ resp. $H^{2}(X / / G, \mathbb{C})$ corresponding to the basis above. These are isomorphic via Kirwan's map; the variables $r_{1}, r_{2}$ are called $q_{1}, q_{2}$ in most of the mirror symmetry literature but we wish to avoid confusion with the universal Novikov parameter $q$. The mirror transformation is

$$
s_{1}=r_{1} /\left(1+r_{1}\right)^{2}, \quad s_{2}=r_{2}\left(1+r_{1}\right) ;
$$

the quantum Kirwan map is obtained from inverting this coordinate transformation and inserting suitable powers of $q$ in the power series expansion, determined by the symplectic class $\omega$. Here we set $q=1$ for simplicity; if $\log \left(r_{i}\right)=\log \left(s_{i}\right)+g_{i}\left(r_{1}, r_{2}\right), \log (r)=$ $\left(\log \left(r_{1}\right), \log \left(r_{2}\right)\right)$ then

$$
\kappa_{X}^{G}(\log (s))=\log (r)=\log (s)+g(s)
$$


so that the "quantum correction" to the Kirwan map is $g(s)$. The image of the divisors classes under the linearized Kirwan map are given by differentiating the mirror transformation. If $\tilde{D}_{j}=D_{\omega} \kappa_{X}^{G}\left(\mu_{j}\right)$, where $\mu_{j}$ is the $j$-th weight, then

$$
\tilde{D}_{1}=D_{1}, \quad \tilde{D}_{2}=D_{2}+2 \frac{r_{1}}{1-r_{1}} D_{2}, \quad \tilde{D}_{3}=D_{3}-\frac{r_{1}}{1-r_{1}} D_{2}, \quad \tilde{D}_{4}=D_{4}-\frac{r_{1}}{1-r_{1}} D_{2} ;
$$

these are called Batyrev elements in González-Iritani [26]. It was noted in Guest [30] for semi-Fano toric varieties, and Iritani [34, Section 5], González-Iritani [26, Example 3.5] these elements satisfy the Batyrev relations with respect to the variables $s_{1}, s_{2}$, which for $d=\left(d_{1}, d_{2}\right) \in \mathbb{Z}_{\geq 0}^{2}, d_{2}-2 d_{1} \geq 0 \mathrm{read}$

$$
\tilde{D}_{1}^{d_{2}} \star_{\log (s)} \tilde{D}_{2}^{d_{2}-2 d_{1}} \star_{\log (s)} \tilde{D}_{3}^{d_{1}} \star_{\log (s)} \tilde{D}_{4}^{d_{1}}=s_{1}^{d_{1}} s_{2}^{d_{2}} .
$$

The effect of using the bulk-deformed quantum product $\star_{\log (r(s))}$ instead of the small quantum product $\star_{\log (s)}$ can be computed using the divisor equation, and leads to the replacement of $s_{1}^{d_{1}} s_{2}^{d_{2}}$ by $r_{1}^{d_{1}} r_{2}^{d_{2}}$ on the right-hand-side. Indeed, for any classes $\alpha, \beta \in$ $H(X / / G)$ and basis $\{\gamma\}$ for $H(X / / G)$ with dual basis $\left\{\gamma^{\vee}\right\}$

$$
\begin{aligned}
\alpha \star_{\log (r(s))} \beta & =\sum_{n, d, \gamma} \frac{s_{1}^{d_{1}} s_{2}^{d_{2}}}{n !}\left\langle\alpha, \beta, \gamma^{\vee}, g(s), \ldots, g(s)\right\rangle_{0, d, n+3} \gamma \\
& =\sum_{d, \gamma} s_{1}^{d_{1}} s_{2}^{d_{2}} \exp \left(g_{1}(s) d_{1}+g_{2}(s) d_{2}\right)\left\langle\alpha, \beta, \gamma^{\vee}\right\rangle_{0, d, 3} \gamma \\
& =\sum_{d, \gamma}\left(s_{1} \exp \left(g_{1}(s)\right)^{d_{1}}\left(s_{2} \exp \left(g_{2}(s)\right)\right)^{d_{2}}\left\langle\alpha, \beta, \gamma^{\vee}\right\rangle_{0, d, 3} \gamma\right. \\
& =\sum_{d, \gamma}\left(s_{1} \exp \left(g_{1}(s)\right)\right)^{d_{1}}\left(s_{2} \exp \left(g_{2}(s)\right)\right)^{d_{2}}\left\langle\alpha, \beta, \gamma^{\vee}\right\rangle_{0, d, 3} \gamma \\
& =\sum_{d, \gamma} r_{1}^{d_{1}} r_{2}^{d_{2}}\left\langle\alpha, \beta, \gamma^{\vee}\right\rangle_{0, d, 3} \gamma \\
& =\left.\left(\alpha \star_{\log (s)} \beta\right)\right|_{y=r .}
\end{aligned}
$$

Thus for the deformed product the Batyrev elements satisfy the Batyrev relation

$$
\tilde{D}_{1}^{d_{2}} \star_{\log (r(s))} \tilde{D}_{2}^{d_{2}-2 d_{1}} \star_{\log (r(s))} \tilde{D}_{3}^{d_{1}} \star_{\log (r(s))} \tilde{D}_{4}^{d_{1}}=r_{1}(s)^{d_{1}} r_{2}(s)^{d_{2}}
$$

which is a special case of Proposition 3.5. It would interesting to derive the formula for $\kappa_{X}^{G}$ above directly from the geometric definition of the quantum Kirwan map.

Motivated by considerations from mirror symmetry, Givental [24] and later Hori-Vafa [31], proposed a description of the quantum cohomology in terms of the Jacobian ring of functions on the critical locus of a certain function, arising as the Landau-Ginzburg potential of the mirror sigma model. In particular, Givental [24] proved an isomorphism of the quantum cohomology of a smooth Fano toric variety with the Jacobian ring.

Proposition 3.7. (Isomorphism of the Batyrev ring with the Jacobian ring of the naive potential)

$$
Q H_{G}(X) / Q S R_{X, G} \rightarrow \operatorname{Jac}\left(W_{X, G}\right),\left[\mu_{j}\right] \mapsto\left[q^{\omega_{j}} y_{j}\right], j=1, \ldots, k
$$


is well-defined and induces an isomorphism.

Proof. Without the Novikov field, the result is Iritani $[33,3.9]$ : the linear relations among the weights for $\mathfrak{g}$ on $X$ correspond to the relations on the coordinate ring of $\tilde{T}^{\vee}$ given by the derivatives of the Landau-Ginzburg potential $W_{X, G}$. Any such relation is of the form

$$
\sum_{i=1}^{k} \mu_{i}\left\langle\lambda, \nu_{i}\right\rangle=0
$$

for some $\lambda \in \mathfrak{t}$. Furthermore the quantum Stanley-Reisner relations $Q S R_{X, G}$ correspond to the relations on the various coordinates on the big dual torus $\tilde{G}^{\vee}$ restricted to $\iota_{\omega} \tilde{T}^{\vee}$ :

$$
\begin{aligned}
\prod_{\left\langle\mu_{j}, d\right\rangle>0} \mu_{j}^{\left\langle\mu_{j}, d\right\rangle} & \mapsto \prod_{\left\langle\mu_{j}, d\right\rangle>0} q^{\omega_{j}\left\langle\mu_{j}, d\right\rangle} y_{j}^{\left\langle\mu_{j}, d\right\rangle} \\
& =q^{\sum_{\left\langle\mu_{j}, d\right\rangle>0} \omega_{j}\left\langle\mu_{j}, d\right\rangle} \prod_{\left\langle\mu_{j}, d\right\rangle>0} y_{j}^{\left\langle\mu_{j}, d\right\rangle} \\
q^{\langle d, \omega\rangle} \prod_{\left\langle\mu_{j}, d\right\rangle<0} \mu_{j}^{\left\langle\mu_{j}, d\right\rangle} & \mapsto q^{\langle d, \omega\rangle} \prod_{\left\langle\mu_{j}, d\right\rangle<0} q^{\omega_{j}\left\langle\mu_{j}, d\right\rangle} y_{j}^{\left\langle\mu_{j}, d\right\rangle} \\
& =q^{d+\sum_{\left\langle\mu_{j}, d\right\rangle<0} \omega_{j}\left\langle\mu_{j}, d\right\rangle} \prod_{\left\langle\mu_{j}, d\right\rangle<0} y_{j}^{\left\langle\mu_{j}, d\right\rangle} .
\end{aligned}
$$

The quantities (15) and (17) are equal since

$$
\prod_{j=1}^{k} y_{j}^{\mu_{j}}=1, \quad \sum_{j=1}^{k} \omega_{j}\left\langle\mu_{j}, d\right\rangle=\langle\omega, d\rangle .
$$

The claimed isomorphism follows.

To compute the dimension of the Jacobian ring we recall the following theorem of Kouchnirenko's [39, 3] describing the number of critical points of a polynomial in several variables. Consider a function given by restricting a finite sum of monomials on $\tilde{G}^{\vee}$ to $\tilde{T}^{\vee}$ :

$$
W: \tilde{T}^{\vee} \rightarrow \mathbb{C}, \quad y \mapsto \sum_{\lambda \in \tilde{\mathfrak{g}}_{\mathbb{Z}}} c_{\lambda} y^{\lambda}, \quad c_{\lambda} \in \mathbb{C} .
$$

Let $\pi: \tilde{\mathfrak{t}}_{\mathbb{Z}} \rightarrow \mathfrak{t}_{\mathbb{Z}}$ denote the projection onto the free part $\mathfrak{t}_{\mathbb{Z}}$ of $\tilde{\mathfrak{t}}_{Z}$.

Definition 3.8. $\quad$ (a) (Newton polytope) The convex polyhedron

$$
\Delta(W):=\operatorname{hull}\left\{\pi(\lambda) \in \mathfrak{t}_{\mathbb{Z}}, c_{\lambda} \neq 0\right\}
$$

is the Newton polytope of $W$.

(b) (Non-degeneracy at infinity) The function $W$ is non-degenerate at infinity if for any face $F \subset \Delta(W)$, the face polynomial

$$
W_{F}: T^{\vee} \rightarrow \mathbb{C}, \quad y \mapsto \sum_{\lambda \in F} c_{\lambda} y^{\lambda}
$$


has no critical points.

(c) (Multiplicity of a critical point) The multiplicity of an isolated critical point $y \in$ $\operatorname{Crit}(W)$ is the intersection multiplicity of $\mathrm{d} W(y)$ at 0 as in Fulton [22, Lemma 12.1].

Theorem 3.9. (Kouchnirenko theorem) Suppose that $W: T^{\vee} \rightarrow \mathbb{C}$ is non-degenerate at infinity, $\left\{\lambda \in \mathfrak{t}_{\mathbb{Z}}, c_{\lambda} \neq 0\right\}$ generate $\mathfrak{t}_{\mathbb{Z}}$, an $\left\{\lambda-\mu, c_{\lambda} \neq 0, c_{\mu} \neq 0\right\}$ generate $\mathfrak{t}_{\mathbb{Z}}$. Then the number \# $\operatorname{Crit}(W)$ of zeroes of $\mathrm{d} W$ counted with multiplicity is equal to

$$
\# \operatorname{Crit}(W)=\operatorname{dim}(T) ! \operatorname{Vol}(\Delta(W)) \# \Gamma \text {. }
$$

Sketch of proof. We sketch the idea of proof, following the survey of Atiyah [3] who reduces the equality to a computation of the volume of a certain toric variety. It suffices to consider the case that the generic stabilizer is $\Gamma=\{1\}$, by considering the components of $\tilde{T}^{\vee}$ separately. Consider the closure of an orbit of $T^{\vee}$ on $\mathbb{P}\left(\oplus_{c_{\lambda} \neq 0} \mathbb{C}_{\lambda}\right)$ of the sum of weight spaces $C_{\lambda}$ with non-zero coefficient $c_{\lambda}$. The function $W: T^{\vee} \rightarrow \mathbb{C}$ extends to a section on the the hyperplane bundle $\mathcal{O}(1)$. The critical locus $\operatorname{Crit}(W)$ is a subset of the intersection of a collection of hyperplanes $H_{j} \subset \mathbb{P}\left(\oplus_{c_{\lambda} \neq 0} \mathbb{C}_{\lambda}\right)$ defined by $y_{j} \partial_{y_{j}} W=0$. The non-degeneracy condition implies that all intersection points $y \in \operatorname{Crit}(W)$ occur in the open torus orbit. It follows that \# Crit $(W)$ equals the degree of the toric variety $X(W)$ associated to $\Delta(W)$. A standard computation shows that the Duistermaat-Heckman measure $\Phi_{*} \operatorname{Vol}_{X(W)} \in$ $\mathcal{D}^{\prime}\left(\mathfrak{t}_{\mathbb{R}}^{\vee}\right)$, the push-forward of the measure $\operatorname{Vol}_{X(W)}$ defined by the Fubini-Study form on $X(W)$ to $\mathfrak{t}_{\mathbb{R}}^{\vee}$, is the characteristic measure $\mu_{\Delta(W)}$ of the polytope $\Delta(W)$. Hence

$$
\begin{aligned}
\# \operatorname{Crit}(W) & =\int_{X(W)} \operatorname{Eul}\left(\mathcal{O}_{X(W)}(1)^{\oplus \operatorname{dim}(T)}\right) \\
& =\operatorname{dim}(T) ! \int_{X(W)} \exp \left(\operatorname{Eul}\left(\mathcal{O}_{X(W)}(1)\right)\right) \\
& =\operatorname{dim}(T) ! \operatorname{Vol}(\Delta(W)) .
\end{aligned}
$$

We apply Kouchnirenko's Theorem 3.9 to the potential. Let $\tilde{T}_{g}^{\vee} \cong \tilde{T}^{\vee}$ denote the fiber of $\tilde{G}^{\vee} \rightarrow G^{\vee}$ over $g \in G$ in (6). Let

$$
W_{X, G, g}: \tilde{T}_{g}^{\vee} \rightarrow \mathbb{C}, \quad y \mapsto \sum_{j=1}^{k} y_{j}
$$

denote the restriction of the sum of coordinate functions $y_{j}, j=1, \ldots, k$ to $\tilde{G}_{g}^{\vee}$. Let $\operatorname{Jac}\left(W_{X, G, g}\right)$ be the coordinate ring of $\operatorname{Crit}\left(W_{X, G, g}\right)$. Let $G^{\vee}$,o be the space of parameters $g \in G^{\vee}$ for which $W_{X, G, g}$ is non-degenerate at infinity. Let $\Delta_{X / / G}^{\vee}$ given as the convex hull of the normal vectors $\nu_{j}$ of facets of $\Delta_{X / / G}$. Then

Lemma 3.10. (Iritani [33, Propositions 3.7,3.10]) $G^{\vee, \circ}$ is a Zariski-open subset of $G^{\vee}$, and for $g \in G^{\vee, \circ}$ the number \# $\operatorname{Crit}\left(W_{X, G, g}\right)$ of critical points of $W_{X, G, g}$, counted with multiplicity is equal to

$$
\# \operatorname{Crit}\left(W_{X, G, g}\right)=\# \Gamma \operatorname{dim}(T) ! \operatorname{Vol}\left(\Delta_{X / / G}^{\vee}\right)
$$


Furthermore, the set of points $G^{\vee, \circ} \subset G^{\vee, \circ}$ where the critical points are non-degenerate is open and dense.

We now compare the number of critical points to the dimension of the quantum cohomology. For each cone $C$ of maximal dimension in the fan $\mathcal{C}(X / / G)$, let $\Sigma(C) \subset \mathfrak{t}_{\mathbb{R}}$ denote the simplex spanned by its generating vectors $\mu_{j}$ and the origin 0 , and $\operatorname{Vol}(\Sigma(C)) \in(0, \infty)$ its volume. An example is shown in Figure 2, where $X / / G$ is a Hirzebruch surface, the dual polytope to $\Delta_{X / / G}$ contains four simplices of volume $1 / 2$, each contributing one to the Euler characteristic $\chi(X / / G)=4$, but these simplices do not cover the dual polytope and so the dual polytope is "bigger than expected".

The following computation of the Euler characteristic the quantum cohomology of a toric stack can be found in Iritani [34] or less explicitly in the earlier paper of BorisovChen-Smith [7]:

Proposition 3.11. [34, Chapter 5] We have

$$
\operatorname{dim} Q H(X / / G)=\operatorname{dim}(X / / G) ! \sum_{C \in \mathcal{C}(X / / G)} \operatorname{Vol}(\Sigma(C))
$$

where the sum is over cones of maximal dimension.

Proof. Since the odd cohomology vanishes we have

$$
\operatorname{dim}(Q H(X / / G))=\chi\left(I_{X / / G}\right)=\chi\left(I_{X / / G}^{T}\right)=\chi\left(I_{(X / / G)^{T}}\right) .
$$

The correspondence between fixed points and cones of maximal dimension proves the identity in the case of smooth toric varieties. In the stack case one uses in addition that the order of the stabilizer $T_{[x]}$ at the fixed point $[x] \in X / / G$ corresponding to $C$ is

$$
\# T_{[x]}=\operatorname{dim}(X / / G) ! \operatorname{Vol}(\Sigma(C))
$$

see $[34,5.10]$.

Corollary 3.12. ([33, 3.10], $[34,5.10])$ The order of $\operatorname{Crit}\left(W_{X, G, g}\right)$ for general $g$ is at least $\operatorname{dim}(Q H(X / / G))$, with equality if and only if $c_{1}(X / / G) \geq 0$.

Because the dimensions of $Q H(X / / G)$ and $\operatorname{Jac}\left(W_{X, G, g}\right)$ for generic $g$ do not match in the non-semi-positive case, see Corollary 3.12, there must be additional relations, that is, generators in the kernel of the map $Q H_{G}(X) \rightarrow Q H(X / / G)$ in (13). Several authors suggested, and Iritani [34] and Fukaya et al [21] proved in the case of toric manifolds, that the additional relations in the kernel of (13) correspond to functions on the critical points $y \in \operatorname{Crit}\left(W_{X, G}\right)$ outside of the moment polytope $\Delta_{X / / G}$. These extra generators can be removed by using the formal version of the Jacobian ring of the potential introduced in Definition 1.13 (a).

Proposition 3.13. (Isomorphism of formal Batyrev and Jacobian rings) The map from $T_{\omega} Q H_{G}(X) / Q S R_{X, G}$ to $\operatorname{Jac}\left(W_{X, G}\right)$ of Proposition 3.7 extends to an isomorphism from $T_{\omega} \widehat{Q H}_{G}(X) / \widehat{Q S R}_{X}^{G}$ to $\mathrm{Jac}_{+}\left(W_{X, G}\right)$. 

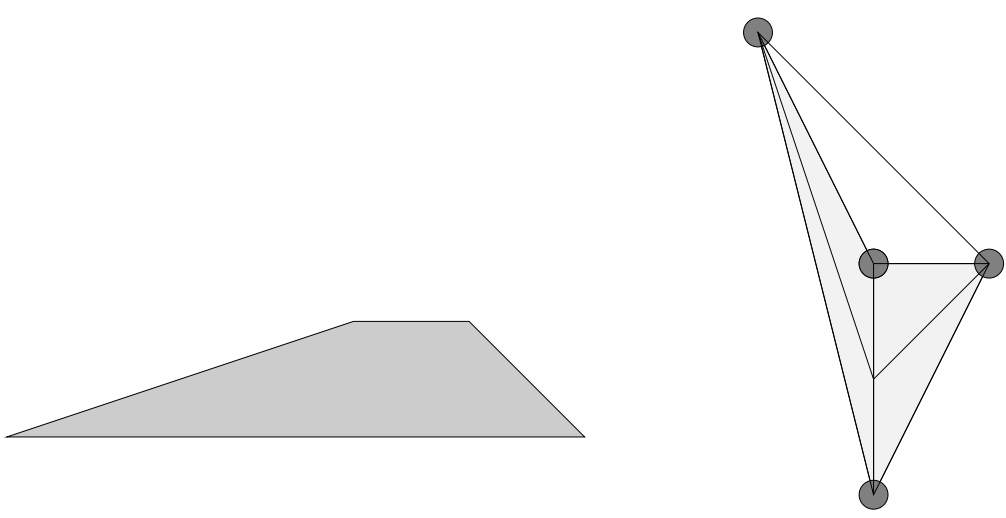

Figure 2. A polytope whose dual polytope has too much volume

Proof. It suffices to check that the degrees giving the filtration used to define completions on both $T_{\omega} Q H_{G}(X) / Q S R_{X, G}$ and $\operatorname{Jac}\left(W_{X, G}\right)$ of Proposition agree. The degree of each weight $\mu_{i} \in Q H_{G}(X)$ is one, while the degree of its image $q^{\omega_{i}} \tilde{g}_{i}$ in the grading of the Jacobian ring given by (6) is also one, by definition. Extension of the isomorphism to the completions $T_{\omega} \widehat{Q H}_{G}(X) / \widehat{Q S R}_{X}^{G}$ and $\mathrm{Jac}_{+}\left(W_{X, G}\right)$ follows.

We now explore the meaning of the positive part of the critical locus more geometrically, in terms of the moment map.

Definition 3.14. (Tropical moment map) The tropical moment map is the map obtained by taking $q$-valuations

$$
\Psi: \tilde{T}^{\vee}(\Lambda) \rightarrow \mathfrak{t}_{\mathbb{R}}^{\vee}, \quad\left(y_{1}, \ldots, y_{k}\right) \mapsto\left(\operatorname{val}_{q}\left(y_{1}\right), \ldots, \operatorname{val}_{q}\left(y_{k}\right)\right) .
$$

where $\mathfrak{t}_{\mathbb{R}}^{\vee}$ is considered a subspace of $\tilde{\mathfrak{g}}_{\mathbb{R}}^{\vee}$ via (6).

Definition 3.15. (Minimal facets) Let $\lambda \in \mathfrak{t}_{\mathbb{R}}^{\vee}$. Let

$$
I(\lambda)=\left\{i \in\{1, \ldots, k\} \mid\left\langle\lambda, \nu_{i}\right\rangle+\omega_{i}=\inf \left\{\left\langle\lambda, \nu_{j}\right\rangle+\omega_{j} \in \mathbb{R}, \quad j=1, \ldots, k\right\}\right.
$$

denote the indices of the "closest" facets to $\lambda$. More generally, for any subspace $\mathfrak{s} \subset \mathfrak{t}_{\mathbb{R}}^{\vee}$, denote by $I(\lambda, \mathfrak{s})$ the set of facets minimal for $\lambda$ among those with $\nu_{j} \mid \mathfrak{s} \neq 0$.

Remark 3.16. For a generic point $\lambda \in \mathfrak{t}_{\mathbb{R}}^{\vee}$, there will be a unique closest facet so $I(\lambda)$ will have order 1 . Each point $\lambda$ on the boundary of $\Delta_{X / G}$ has minimal facets $I(\lambda)$ equal to the set of facets of $\Delta_{X / / G}$ containing $\lambda$. The same holds in a neighborhood of the boundary $\partial \Delta_{X / / G}$, by continuity. For examples of points $\lambda$ with more than $\operatorname{dim}(T)$ minimal facets, see Figure 3.

Proposition 3.17. Let $y \in \operatorname{Crit}\left(W_{X, G}\right)$ be a critical point. The tropical moment map $\zeta=\Psi(y) \in \mathfrak{t}_{\mathbb{R}}^{\vee}$ has the property that for any $\mathfrak{s}$ with $I(\zeta, \mathfrak{s}) \neq \emptyset$, the normal vectors $\nu_{j}, j \in I(\zeta, \mathfrak{s})$ are linearly dependent after restriction to $\mathfrak{s}$ in $\mathfrak{t}_{\mathbb{R}}^{\vee}$. 
Proof. We take the derivative of the potential: For $\lambda \in \mathfrak{s}$ and $y \in \operatorname{Crit}\left(W_{X, G}\right)$

$$
0=\partial_{\lambda} W_{X, G}(y)=\sum_{j=1}^{k} q^{\omega_{j}} y^{\nu_{j}}\left\langle\nu_{j}, \lambda\right\rangle .
$$

In particular the leading order powers of $q$ in (19) must cancel. Thus

$$
\forall \lambda, \sum_{j \in I(\zeta, \mathfrak{s})} y^{\nu_{j}}\left\langle\nu_{j}, \lambda\right\rangle=0 \quad \text { so } \sum_{j \in I(\zeta, \mathfrak{s})} \operatorname{val}_{q}\left(y^{\nu_{j}}\right) \nu_{j}=0
$$

so the vectors $\nu_{j}$ are dependent after restriction to the subspace $\mathfrak{s}$.

Example 3.18. (a) (Tropical moment map for critical locus for a product of projective lines) Let $X=\mathbb{C}^{4}$ with $G=\left(\mathbb{C}^{\times}\right)^{2}$ acting with weights $(1,0),(1,0)$, $(0,1),(0,1)$. Consider the reduction at $\omega=(2,1)$ then $X / / G=\mathbb{P}^{1} \times \mathbb{P}^{1}$ with moment polytope $[4,0] \times[2,0]$ and normal vectors $(1,0),(-1,0),(0,1),(0,-1)$. The critical points are $\left(y_{1}, y_{2}\right)=\left( \pm q^{2}, \pm q\right)$ which have valuations (leading order $q$-powers) $(2,1)$, all mapping to the barycenter of the moment polytope. We have $I(\lambda)=\{3,4\}$, the facets closest to the critical point. If $\mathfrak{s}=\operatorname{span}(1,0)$ then $I(\lambda, \mathfrak{s})=\{1,2\}$. Note that the vectors $\nu_{j}, j \in I(\lambda)$ or $I(\lambda, \mathfrak{s})$ are dependent.

(b) (Tropical moment map for critical locus for a family of toric surfaces) Suppose that $X=\mathbb{C}^{5}$ with $G=\left(\mathbb{C}^{\times}\right)^{3}$ acting with weight matrix

$$
\left[\begin{array}{rrrrr}
1 & 1 & 0 & 0 & 0 \\
0 & 0 & 1 & 1 & 0 \\
-1 & 0 & -1 & 0 & 1
\end{array}\right]
$$

For a suitable choice of $\omega$ the quotient $X / / G$ is the blow-up of projective lines $\mathbb{P}^{1} \times \mathbb{P}^{1}$ at a fixed point, say $([1,0],[1,0])$, with moment polytope

$$
\Delta_{X / / G}=\left\{\left(\mu_{1}, \mu_{2}\right) \in[0,4] \times[0,2] \mid \mu_{1}+\mu_{2} \geq \epsilon\right\} .
$$

For $\epsilon<1$, there are two possible values of $\Psi$ on $\operatorname{Crit}\left(W_{X, G}\right)$ : one critical point maps to $(\epsilon, \epsilon)$, while four other critical points map to $(2,1)$. For $1<\epsilon$, one critical point maps to $(2-\epsilon, \epsilon)$. The others map to $((3+\epsilon) / 2,1)$. See Figure 3. The case $\epsilon=1$ is special: in this case, one can obtain a line segment of critical values $\Psi(y), y \in \operatorname{Crit}\left(W_{X, G}\right)$ by varying the "bulk deformation", see [19]. This is shown as a dotted line connecting the two critical values in the Figure 3.

Proposition 3.19. A point $y \in \operatorname{Crit}\left(W_{X, G}\right)$ lies in $\operatorname{Crit}_{+}\left(W_{X, G}\right)$ iff $\Psi(y) \in \Delta_{X / / G}$.

Proof. It suffices to consider the case $\Gamma$ trivial. The $j$-th coordinate of $y$ under the embedding $T^{\vee} \rightarrow \tilde{G}^{\vee}$ is $y_{j}=y^{\nu_{j}}$. The shift $y_{j} q^{\omega_{j}}$ has $q$-valuation $\left\langle\nu_{j}, \Psi(y)\right\rangle+\omega_{j}$. Thus $y_{j} q^{\omega_{j}}$ goes to zero as $q \rightarrow 0$ iff $\left\langle\nu_{j}, \Psi(y)\right\rangle>-\omega_{j}$.

Remark 3.20. By the results of [20], [19], [53], the image of $\operatorname{Crit}_{+}\left(W_{X, G}\right)$ in $\mathfrak{t}_{\mathbb{R}}^{\vee}$ consists of moment values such that the corresponding Lagrangian moment fiber is Hamiltonian non-displaceable, see Section 5. 


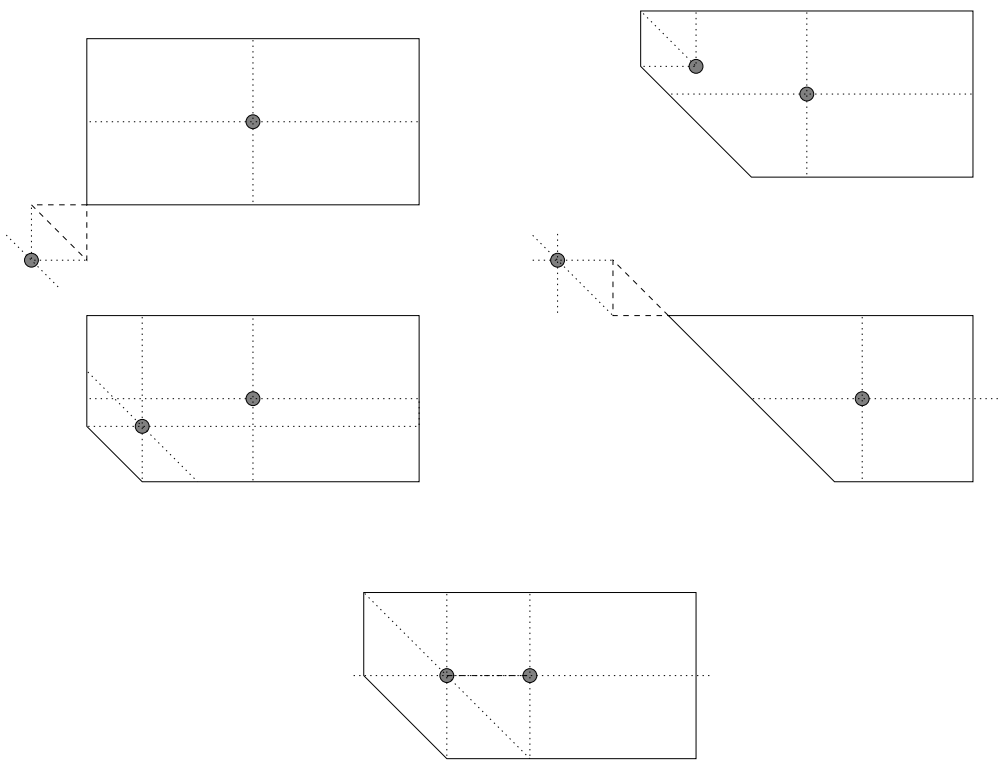

Figure 3. Values of the tropical moment map on the critical locus for a family of toric surfaces

\section{Dimensional equality Via a toric minimal model Program}

In this section we show that the linearized quantum Kirwan map is injective after passing to the formal completion and modding out by the quantum Stanley-Reisner ideal. By the surjectivity result in Theorem 2.6 it suffices to show the equality of dimensions

$$
\operatorname{dim} Q H(X / / G)=\operatorname{dim} \operatorname{Jac}_{+}\left(W_{X, G}\right) .
$$

In the case that $X / / G$ is Fano and minimally presented as a quotient of $X$ by $G$ (that is, every weight space in $X$ defines a prime divisor of $X / / G$ ) this is a consequence of Kouchnirenko's theorem, see Corollary 3.12.

To reduce to the Fano case, we apply the toric minimal model program introduced by M. Reid [45]. More precisely, we vary the Kähler class $[\omega]$ by a multiple $-t c_{1}(X / / G)$ of the canonical class $c_{1}(X / / G)$ until we obtain a Fano fibration, showing that the wall-crossings on both sides of (20) are the same. We wish to emphasize that, although we are using the language of toric minimal models, in fact all of our results are completely combinatorial, that is, could be phrased entirely in terms of fans. However, we find the geometric story accompanying the combinatorics rather helpful. First, recall the general phenomenon of wall-crossing in the context of geometric invariant theory quotients, as in Dolgachev-Hu [17] and Thaddeus [47] in which flips occur as the polarization defining the quotient is varied.

Notation 4.1. (Family of git quotients) Let $\omega_{t} \in H_{G}^{2}(X, \mathbb{Q}), t \in[0,1]_{\mathbb{Q}}$ be an affine linear path of Kähler classes, corresponding to a path of rational polarizations (ample $G$-line 
bundles) $L_{t} \rightarrow X$. For any $t \in[0,1]_{\mathbb{Q}}$ let

$$
X^{t, \mathrm{ss}}=\bigcup_{k>0, s \in H^{0}\left(X, L_{t}^{k}\right)^{G}}\{s \neq 0\}
$$

be the semistable locus, and assume that $G$ acts with finite stabilizers on $X^{t, \mathrm{ss}}$ for $t=0,1$. Then the $G$ acts with finite stabilizers on $X^{t, \text { ss }}$ for generic $t \in[0,1]$. For such $t$ denote by

$$
X / /{ }_{t} G:=X^{t, \mathrm{ss}} / G
$$

the stack-theoretic git quotient with respect to the corresponding polarization. The stack $X / /{ }_{t} G$ is a smooth proper Deligne-Mumford stack with projective coarse moduli space.

Proposition 4.2. (Wall-crossing for git quotients) With $G, X, L_{t}$ as above.

(a) (Walls) there exists a finite collection $t_{1}, \ldots, t_{n} \in(0,1)$ of singular values a.k.a walls such that there exist semistable points that are not stable;

(b) (Chambers) the isomorphism class of the quotient $X / /{ }_{t} G$ is independent of $t$ for $t \in\left(t_{j}, t_{j+1}\right), j=1, \ldots, n-1$;

(c) (Wall-crossing) Suppose that stable=semistable for the $G$-action on $\mathbb{P}\left(L_{0} \oplus L_{1}\right)$. Then as $t$ passes through a singular value $t_{j}$, the quotient $X / /{ }_{t} G$ goes through a stacky-weighted blow-down and blow-up over a center $Z \subset X / / t_{j} G$.

See Figure 4 for an example of the change in moment polytopes under such a variation; the toric case is discussed further in [45], [42, Chapter 14].

Remark 4.3. Suppose that $X / /{ }_{t} G$ is a family of toric quotients and $t \in(0, \infty)$ a singular value. The singularity in $X / /{ }_{t} G$ is necessarily created by an intersection of facets of $\Delta\left(X / /{ }_{t} G\right)$ with linearly dependent normal vectors $\nu_{j}, j \in I(t)$. Let

$$
Z=\bigcap_{j \in I(t)} D_{j} \subset X / /{ }_{t} G
$$

denote the intersection of the prime divisors $D_{j}, j \in I(t)$. Denote the morphisms to the singular quotients on the level of coarse moduli spaces

$$
\pi_{ \pm}: X / / t_{ \pm} G \rightarrow X / / t G
$$

Denote the exceptional loci $E_{ \pm}:=\pi_{ \pm}^{-1}(Z)$. The restrictions of $\pi_{ \pm}$to the exceptional loci

$$
\pi_{ \pm} \mid E_{ \pm}: E_{ \pm} \rightarrow Z
$$

are fiber bundles. The fibers $\pi_{ \pm}^{-1}(z), z \in Z$ are weighted projective stacks $\mathbb{P}\left(I_{ \pm}\right)$corresponding to the subset of weights

$$
I_{ \pm} \subset\left\{\nu_{j}, j \in I(t)\right\}
$$

corresponding to prime divisors not containing $E_{ \pm}$. The fan $\mathcal{C}(Z)$ of the center $Z$ is given by the projections of the cones $\mathcal{C}_{Z}\left(X / /{ }_{t} Z\right)$ of $\mathcal{C}(X / / G)$ corresponding to orbits meeting $Z$ to the Lie algebra $\mathfrak{g}^{\prime}=\mathfrak{g} / \operatorname{span}\left(\nu_{1}, \ldots, \nu_{k}\right)$. The morphism of toric varieties $\pi_{ \pm}$corresponds to a morphism of fans

$$
\mathcal{C}\left(X / / t_{ \pm} G\right) \rightarrow \mathcal{C}\left(X / /{ }_{t} G\right)
$$


that is an isomorphism over the complement of the cones in $\mathcal{C}\left(X / /{ }_{t} G\right)$ containing $C(Z) \in$ $C\left(X / /{ }_{t} G\right)$.

We will be particularly interested in the variation of git quotient for toric quotients corresponding to the anti-canonical class.

Notation 4.4. (Anticanonical variations) A path $\omega_{t} \in H_{G}^{2}(X), t \in[0, T]$ is an anticanonical variation of symplectic class if $\frac{d}{d t} \omega_{t}=-c_{1}^{G}(T X)$, see Figure 4 . Note that since $T X$ is a sum of line bundles with $\tilde{G}$-weights $\epsilon_{i}$,

$$
c_{1}^{\tilde{G}}(T X)=\sum_{i=1}^{k} \epsilon_{i} .
$$

The variation of symplectic class $\omega$ can be taken to be

$$
\left(\omega-t c_{1}^{G}(T X)\right)_{i}=\omega_{i}-t, \quad i=1, \ldots, k .
$$

Hence the facets of the polytope $\Delta_{X / / t}$ "vary at the same rate":

$$
\Delta_{X / / t G}=\left\{\mu \in \mathfrak{t}_{\mathbb{R}}^{\vee} \mid\left\langle\mu, \nu_{j}\right\rangle \geq-\omega_{j}+t, j=1, \ldots, k\right\} .
$$

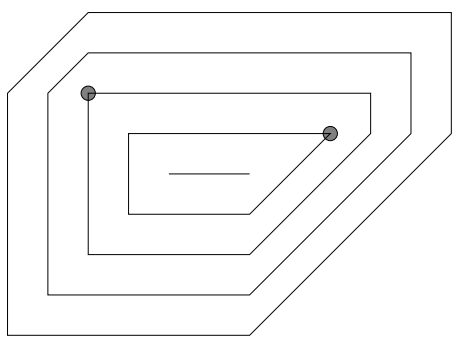

Figure 4. Polytopes for a toric minimal model program

The sequence of a toric varieties obtained in this way is a special case of the minimal model program described in the toric case by Reid [45]; see [15, Chapter 15] for more references. An example of the family of polytopes $\Delta_{X / / t} G$ obtained in this way is shown in Figure 4; the corresponding fans $\mathcal{C}\left(X / /{ }_{t} G\right)$ are shown in Figure 5.

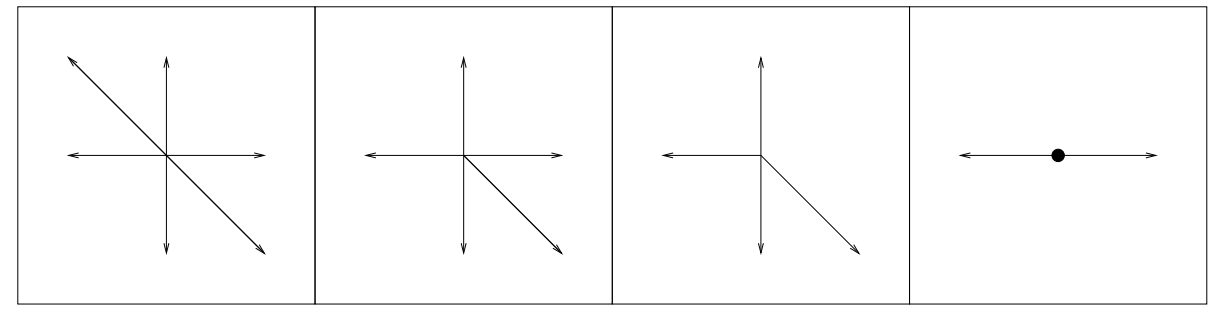

FiguRE 5. Fans for a toric minimal model program

The following is the key property of generic runnings of the toric minimal model program. 
Proposition 4.5. For a generic symplectic class $\omega$, at any singular value $t=t_{1}, \ldots, t_{n}$, given a collection of normal vectors $\nu_{j}, j \in I(t)$ that span a subspace of dimension

$$
\operatorname{dim}\left(\operatorname{span}\left\{n_{j}, j \in(t)\right\}\right)=\ell
$$

the following holds: any point $\mu$ in $\mathfrak{t}^{\vee}$ satisfies at most $\ell+1$ inequalities $\left\langle\mu, \nu_{j}\right\rangle \geq \omega_{j}+t, j \in$ $I(t)$ with strict equality.

Proof. For each subset $I$ such that the vectors $\nu_{j}, j \in I$ span a subspace of $\mathfrak{t}_{\mathbb{R}}$ of size $l$, the space $\mathcal{S}_{I}$ of tuples $(\omega, \lambda, t)$ where such that at least $l+2$ equalities (18) hold is a union of affine subspaces of dimension at most $\operatorname{dim}\left(H_{G}^{2}(X)\right)-1$. The projection $p\left(\mathcal{S}_{I}\right)$ of $\mathcal{S}_{I}$ under $H_{G}^{2}(X) \times \mathfrak{t}_{\mathbb{R}}^{\vee} \times \mathbb{R} \rightarrow H_{G}^{2}(X)$ is a proper affine subspace of codimension at least 1 . Taking the union over all possible subsets $I \subset\{1, \ldots, k\}$ proves the claim.

We will need the following explicit description of the flips arising from variation in the anticanonical direction.

Definition 4.6. (Flipping simplex) Suppose that $\omega_{t}$ is a path of classes as above with $\frac{d}{d t} \omega_{t}=-c_{1}^{G}(X)$. Let $t \in(0, \infty)$ be a singular value, corresponding to an intersection $\cap_{j \in I(t)} F_{j, t} \subset \Delta_{X / / t}$ of facets $F_{j, t}$ with indices $j \in I(t)$ :

$$
F_{j, t}=\left\{\mu \in \Delta_{X / / t} G \mid\left\langle\mu, \nu_{j}\right\rangle=-\omega_{j}+t\right\} .
$$

Let $\Sigma_{t}$ denote the flipping simplex

$$
\Sigma_{t}:=\operatorname{hull}\left(\nu_{j}, j \in I(t)\right) \subset \mathfrak{t}_{\mathbb{R}}
$$

that is the convex hull of normal vectors $\nu_{j}$ corresponding to the facets $F_{j, t}$.

Lemma 4.7. For each singular time $t=t_{1}, \ldots, t_{n}$, the leading order term potential $W_{t}(y)=\sum_{j \in I(t)} y_{j}$, has only non-degenerate critical points.

Proof. We may suppose $\Gamma=\{1\}$ and $I=\{1, \ldots, \ell+1\}$. By the linear dependence assumption, there exist $c_{1}, \ldots, c_{\ell} \in \mathbb{R}$ such that $\nu_{\ell+1}=c_{1} \nu_{1}+\cdots+c_{\ell} \nu_{\ell}$. The equations defining the critical locus $\operatorname{Crit}\left(W_{X, G}\right)$ are the partial derivatives with respect to the local coordinates $y^{\nu_{1}}, \ldots, y^{\nu_{\ell}}$

$$
y^{\nu_{i}}=c_{i} y^{\nu_{\ell+1}}, \quad i=1, \ldots, \ell .
$$

These are solutions to the single equation

$$
z^{c_{1}+\ldots+c_{\ell}+1}=1, \quad z=c_{i} y^{\nu_{\ell+1}}, i=1, \ldots, \ell
$$

and as such are transversally cut out.

Proposition 4.8 (Explicit description of flips for the toric minimal model program). Let $X / / t G$ be as above so that the condition of Proposition 4.5 is satisfied. For each singular value $t$, one of the two possibilities holds:

(a) (Fibration case) Suppose that the flipping simplex $\Sigma_{t}$ of (21) contains the origin 0. Then $X / /{ }_{t} G$ undergoes a Mori fibration with fiber a Fano toric stack $(X / / t G)^{\prime}$ over a toric stack $(X / / t G)^{\prime \prime}$ of lower dimension, and the symplectic class on the fiber is a multiple of the first Chern class $c_{1}\left(\left(X / /{ }_{t} G\right)^{\prime}\right)$. 
(b) (Flip or divisorial contraction case) Suppose that the flipping simplex $\Sigma_{t}$ does not contain 0. Then $X / / t G$ undergoes a flip (resp. divisorial contraction). That is, $X / /{ }_{t} G$ undergoes a stacky-weighted blow-down followed by stacky-weighted blow-up (resp. stacky-weighted blow-down only) over a center $Z \subset X / /{ }_{t} G$.

Proof. The fibration case is straight-forward and left to the reader. For the flip/divisorial contraction case, see [15, Lemma 15.3.11], [42, Proof of Proposition 14-2-11] and especially [42, Figure 14-2-12]. One can give a proof using variation of git as follows: Let $G \times \mathbb{C}^{\times}$ act on $\tilde{X}=X \times \mathbb{C}=\mathbb{C}^{k+1}$ with weights $\tilde{\mu}_{j}=\left(\mu_{j}, 1\right)$ and $(0,1)$ and polarization vector $\tilde{\omega}=(\omega, 0)$. The "master space" given by the quotient $\tilde{X} / / G$ has a residual $\mathbb{C}^{\times}$-action whose quotients are the git quotients $X / / t_{t} G$. The transition times correspond to the fixed point components $Z \subset(\tilde{X} / / G)^{\mathbb{C}^{\times}}$; each is necessarily a subvariety of $X / /{ }_{t} G$ obtained by first restricting to the locus $X^{\chi}$ where $\mathbb{C}^{\times}$acts by a character $\chi$ of $G$ and taking the git quotient. The normal bundle $N$ to $(\tilde{X} / / G)^{\mathbb{C}^{\times}}$splits into the sum of negative resp. positive weight sub-bundles $N_{-}$resp. $N_{+}$, each of which is quotient of the sum of negative resp. positive weights in $X / X^{\chi}$ under the action of $\mathbb{C}^{\times}$. The weighted blow-down and blow-up involved from passing to $X / / t_{-} \mathbb{C}^{\times}$to $X / / t_{+} \mathbb{C}^{\times}$replaces the projectivization $\mathbb{P}_{-}$of the sum of the weight bundles $N_{-}$with the projectivization $\mathbb{P}_{+}$of the sum of the weight bundles $N_{+}$. The claim follows.

An example is shown in Figure 6, continuing that in Figures 4, 5, where the flipping simplices for each step are shaded.

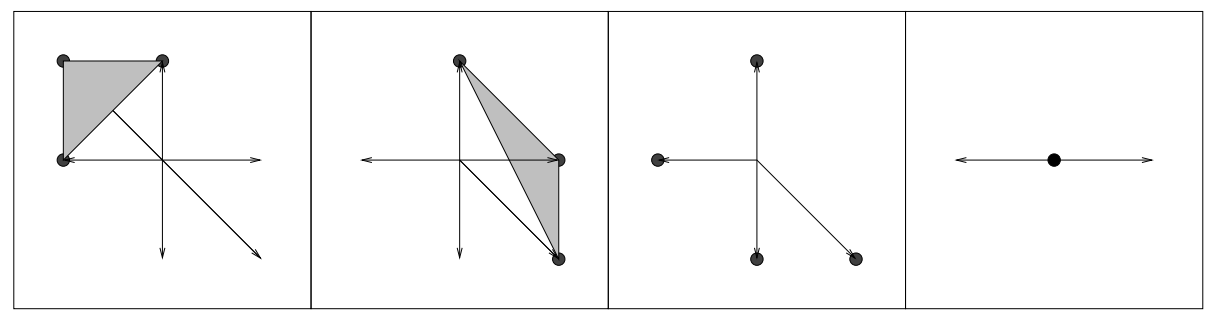

FigURE 6. Flipping simplices for a toric minimal model program

We now describe the change in the critical locus of the potential as the toric stack undergoes an mmp transition. In the fibration case the fan $\mathcal{C}\left(X / / t_{-} G\right)$ admits a morphism to the fan $\mathcal{C}\left(\left(X / / t_{t} G\right)^{\prime}\right)$ of the base, that is, each cone for $X / / t_{-} G$ maps to a cone, possibly of lower dimension, of $\left(X / /{ }_{t} G\right)^{\prime}$; and the cones of $\mathcal{C}\left(X / / t_{-} G\right)$ that map to the origin form the fan of the fiber $\left(X / /{ }_{t} G\right)^{\prime \prime}$. By Proposition 4.5, the fan $\mathcal{C}\left(\left(X / /{ }_{t} G\right)^{\prime}\right)$ for the fiber $\left(X / /{ }_{t} G\right)^{\prime}$ has a minimal number of generators $\nu_{j}$. That is, the corresponding polytope is a simplex $\Delta\left(\left(X / /{ }_{t} G\right)^{\prime}\right)$ and the corresponding toric stack $\left(X / /{ }_{t} G\right)^{\prime}$ is a stacky weighted projective space.

Lemma 4.9. (Critical loci of the Landau-Ginzburg potentials for fibrations) Let $X, G, \omega$ be as above. Suppose that $t \in(0, \infty)$ is a singular value so that $X / /{ }_{t} G$ with potential $W_{X / / t} G$ undergoes a fibration over a toric variety $\left(X / /{ }_{t} G\right)^{\prime}$ of lower dimension with fiber $\left(X / /{ }_{t} G\right)^{\prime \prime}$, 
with potentials $\left.W_{(X / / t} G\right)^{\prime}$ and $\left.W_{(X / / t} G\right)^{\prime \prime}$. Then there is a canonical bijection

$$
\left.\left.\operatorname{Crit}_{+}\left(W_{X / / t_{-} G}\right) \rightarrow \operatorname{Crit}_{+}\left(W_{(X / / t} G\right)^{\prime}\right) \times \operatorname{Crit}_{+}\left(W_{(X / / t} G\right)^{\prime \prime}\right) .
$$

Proof. We may assume that $\Gamma=1$, by treating the components of $\tilde{T}^{\vee}$ individually. The fibration of $X / / t_{-} G$ induces a fibration of tori and dual tori,

$$
1 \rightarrow T^{\prime \prime} \rightarrow T \rightarrow T^{\prime} \rightarrow 1, \quad 1 \rightarrow T^{\vee},^{\prime} \stackrel{p}{\rightarrow} T^{\vee} \stackrel{r}{\rightarrow} T^{\vee},^{\prime \prime} \rightarrow 1
$$

for which we may choose a splitting. We write

$$
W_{X / t_{-} G}=W_{X / / t_{-} G}^{\prime}+W_{X / / t_{-} G}^{\prime \prime}
$$

where

$$
W_{X / t_{-} G}^{\prime \prime}=\sum_{F_{j} \supset \Delta\left(X / / t_{-} G\right)} q^{\omega_{j}} y^{\nu_{j}}
$$

is the sum of terms corresponding to hyperplanes containing the polytope at the singular time, that is, the hyperplanes describing the fiber, and $W_{X / / t_{-} G}^{\prime}$ is the sum of the remaining terms of $W_{X, G}$, corresponding to facets of the base. The leading order terms in $W_{X / / t_{-} G}$ for $y \in \Delta_{X / / t_{-} G}$ are $W_{X / / t_{-} G}^{\prime \prime}$, which restricts to a constant on $T^{\vee,} \subset T^{\vee}$. Thus $\operatorname{Crit}_{+}\left(W_{X / / t_{-} G}\right)$ maps to $\operatorname{Crit}_{+}\left(W_{(X / / t G)^{\prime}}\right)$. The fiber of the projection of $\mathrm{Crit}_{+}\left(W_{X / / t_{-} G}\right)$ onto $\operatorname{Crit}_{+}\left(W_{\left(X / t_{t} G\right)^{\prime}}\right)$ may be identified with $\left.\operatorname{Crit}_{+}\left(W_{(X / t} G\right)^{\prime \prime}\right)$, since $\left.W_{(X / / t}^{\prime \prime} G\right)$ are the nondegenerate terms of leading order, by Proposition 4.13 below.

Remark 4.10. The fibration exact sequence Lemma 4.9 only holds for critical points lying over the interior of the moment polytope. In other words, there is no fibration of $\operatorname{Crit}\left(W_{X / t_{-} G}\right)$ similar to that stated in Lemma 4.9. Indeed suppose that $X / / G$ is a Hirzebruch surface $\mathbb{P}\left(\mathcal{O}_{\mathbb{P}^{1}}(n) \oplus \mathcal{O}_{\mathbb{P}^{1}}\right)$ for some $n \geq 0$. Then $\operatorname{Crit}\left(W_{X / / t_{-} G}\right)$ has order $2+2 n$ for $n \geq 2$ (twice the volume of the polytope with vertices $(-1,0),(0,-1),(1, n)$ ) but $\left.\left.\operatorname{Crit}\left(W_{(X / / t} G\right)^{\prime}\right) \times \operatorname{Crit}\left(W_{(X / / t} G\right)^{\prime \prime}\right)$ has order 4 .

Lemma 4.11. (Dimension lemma for fibrations) Suppose that $X / /{ }_{t} G$ as above with generic initial symplectic class $\omega_{0}$. Let $t \in(0, \infty)$ be a singular value so that $X / / t G$ undergoes a fibration over a toric stack $\left(X / /{ }_{t} G\right)^{\prime}$ of lower dimension with fiber $\left(X / /{ }_{t} G\right)^{\prime \prime}$. Then

(a) $\operatorname{dim}\left(Q H\left(X / / t_{-} G\right)\right)=\operatorname{dim}\left(Q H\left(\left(X / /{ }_{t} G\right)^{\prime}\right)\right) \operatorname{dim}\left(Q H\left(\left(X / /{ }_{t} G\right)^{\prime \prime}\right)\right)$ and

(b) $\left.\operatorname{dim}\left(\operatorname{Jac}_{+}\left(W_{X / / t_{-} G}\right)=\operatorname{dim}\left(\operatorname{Jac}_{+}\left(W_{(X / / t} G\right)^{\prime}\right)\right) \operatorname{dim}\left(\operatorname{Jac}_{+}\left(W_{(X / / t} G\right)^{\prime \prime}\right)\right)$.

Proof. (a) By Proposition 4.8, the cones of $X / / t_{-} G$ of maximal dimension are products of the maximal dimensional cones of $\left(X / /{ }_{t} G\right)^{\prime}$ and $\left(X / /{ }_{t} G\right)^{\prime \prime}$. It follows the sum of the volumes of the maximal dimensional cones of $X / / t_{-} G$ is the product of the corresponding sums for $(X / / t G)^{\prime}$ and $\left(X / /{ }_{t} G\right)^{\prime \prime}$. The conclusion follows from Lemma 3.11. (b) follows from Lemma 4.9.

In order to deal with flips with non-trivial centers with we describe a stage-wise implicit function theorem due that was communicated to us by S. Venugopalan. 
Definition 4.12. A function $W: \tilde{T}^{\vee}(\Lambda) \rightarrow \Lambda$ has a stage-wise non-degenerate critical point $y \in \tilde{T}^{\vee}(\Lambda)$ with exponents $\tau_{1}<\ldots<\tau_{n} \in \mathbb{R}$ if the following holds: There exist decompositions

$$
\tilde{T}^{\vee}(\Lambda)=\tilde{T}_{1}^{\vee}(\Lambda) \times \cdots \times \tilde{T}_{n}^{\vee}(\Lambda), \quad W=W_{1}+\cdots+W_{n}, \quad y=\left(y_{1}, \ldots, y_{n}\right)
$$

where $T_{i}^{\vee}(\Lambda)$ are products of tori with finite groups $\Gamma_{i}$, and

(a) the map $W_{i}: \tilde{T}^{\vee}(\Lambda) \rightarrow \Lambda_{\geq 0}$ factors through the projection onto the first $i$ factors

$$
\pi_{i}: \tilde{T}^{\vee}(\Lambda) \rightarrow \Pi_{j=1}^{i} \tilde{T}_{j}^{\vee}(\Lambda)
$$

(b) the leading order term of $W_{i}$ has $q$-valuation $\tau_{i}$, and

(c) for $i \geq 2$, the point $y_{i} \in \tilde{T}_{i}^{\vee}(\Lambda)$ is a non-degenerate critical point of

$$
\tilde{W}_{i}::=\left.W_{i}\right|_{\tilde{T}_{i}^{\vee}(\Lambda)}: \tilde{T}_{i}^{\vee}(\Lambda) \rightarrow \Lambda
$$

This ends the Definition.

Proposition 4.13. (Stage-wise implicit function theorem) Suppose $W: \tilde{T}^{\vee}(\Lambda) \rightarrow \Lambda$ is a potential function such that every critical point $y \in \mathrm{Crit}_{+}(W)$ is stage-wise non-degenerate. For each decomposition as above $\tilde{T}^{\vee}=\Pi_{i=1}^{n} \tilde{T}_{i}^{\vee}$ there exists a bijection

$$
\prod_{i=1}^{n} \operatorname{Crit}_{+}\left(\tilde{W}_{i}\right) \rightarrow \mathrm{Crit}_{+}(W)
$$

for sufficiently small $\tau_{1}, \ldots, \tau_{n}$.

Proof. Given a critical point for the potentials in each direction, wesolve for a critical point of the full potential order by order, using non-degeneracy of the Hessians. Let $y=\left(y_{1}, \ldots, y_{n}\right) \in \prod_{i=1}^{n} \operatorname{Crit}_{+}\left(\tilde{W}_{i}\right)$ be a critical point of the stage-wise leading order terms. Suppose that the summands of

$$
D_{y} W=\sum_{i=1}^{n} D_{y} W_{i}
$$

have leading order terms divisible by $q^{\tau_{1}+\delta}, \ldots, q^{\tau_{n}+\delta}$ for some $\delta>0$. We solve by taking a Taylor expansion of $D_{y \exp (z)} W$ at $y$

$$
\begin{aligned}
0 & =D_{y \exp (z)} W \\
& =D_{y} W+\frac{1}{2 !} D_{y}^{2} W(z, \cdot)+\frac{1}{3 !} D_{y}^{3} W(z, z, \cdot)+\text { higher order in } z .
\end{aligned}
$$

We work out the details in case $n=2$. The proof extends naturally to higher values of $n$. We claim that we can solve for $z$ satisfying

$$
D_{y} W+\frac{1}{2} D_{y}^{2} W(z, \cdot)=0 .
$$

The operator

$$
\frac{1}{2} D_{y}^{2} W: T_{y} \tilde{T}^{\vee}(\Lambda) \times T_{y} \tilde{T}^{\vee}(\Lambda) \rightarrow \Lambda
$$


after a choice of basis respecting the splitting, has a block matrix representation

$$
\frac{1}{2} D_{y}^{2} W=\left(\begin{array}{cc}
q^{\tau_{1}} G_{11} & q^{\tau_{2}} G_{12} \\
0 & q^{\tau_{2}} G_{22}
\end{array}\right)
$$

where $G_{i j}$ is a $\Lambda_{\geq 0}$-valued matrix. By non-degeneracy $G_{11}$ and $G_{22}$ are invertible. The matrix $G$ has an inverse $G^{-1}:=\left(\begin{array}{cc}q^{-\tau_{1}} G^{11} & q^{-\tau_{1}} G^{12} \\ 0 & q^{\tau_{2}} G^{22}\end{array}\right)$, where for any $i, j, G^{i j}$ is a $\Lambda_{\geq 0^{-}}$ valued matrix. In matrix notation, the solution $z$ is given by

$$
z=\left(\left(D_{y} W\right) G^{-1}\right)^{t} .
$$

Since the leading order terms in $D_{y} W$ are divisible by $\tau_{1}+\delta, \tau_{2}+\delta$, the leading order terms of $z$ have $q$-valuations at least $\delta$. The equation (24) fails to hold only because of the terms quadratic or higher order in $z$. Since the splitting $W=\left(W_{1}, W_{2}\right)$ is divisible by $\left(q^{\tau_{1}}, q^{\tau_{2}}\right)$ and $z$ is divisible by $q^{\delta}$, the term $\frac{1}{3 !} D_{y}^{3}(z, z, \cdot)$ and higher order terms in $(24)$ are divisible by $\left(q^{\tau_{1}+2 \delta}, q^{\tau_{2}+2 \delta}\right)$. Replacing $y$ with $y \exp (z)$ and continuing by induction one obtains a solution to all orders. Conversely, given a stage-wise non-degenerate critical point $y \in \operatorname{Crit}(W)$ we obtain a critical point $y_{i}$ for each $\tilde{W}_{i}$ by projection. Since the $q$-valuation of the critical point $y(q)$ is the minimum of the $q$-valuations of the elements $y_{i}(q)$, the element $y(q)$ has a limit as $q \rightarrow 0$ iff the elements $y_{i}(q)$ also have a limit as $q \rightarrow 0$.

Lemma 4.14. For generic $[\omega] \in H_{G}^{2}(X, \mathbb{Q})$, every critical point $y \in \operatorname{Crit}\left(W_{t}\right)$ for $t \in \mathbb{R}$ is stage-wise non-degenerate.

Proof. The proof is an application of Proposition 4.5 and Lemma 4.7. For any $\epsilon \in \mathbb{R}$ let $I(\mu, \epsilon)$ denote the set of indices of facets at distance $\epsilon$ from $\mu$ :

$$
I(\mu, \epsilon):=\left\{i \in\{1, \ldots, k\} \mid\left\langle\mu, \nu_{i}\right\rangle+\omega_{i}=\epsilon\right\} .
$$

The dimension count in Proposition 4.5 shows that for generic $\omega_{i}$

$$
\# I(\mu, \epsilon) \leq 1+\operatorname{dim}\left(\operatorname{span}\left(\nu_{i}\right), i \in I(\mu, \epsilon)\right) .
$$

Let $\epsilon=\epsilon_{1}$ be the minimum value for which the vectors $\nu_{i}, i \in I(\mu, \epsilon)$ is linearly dependent. The sum of the terms $\sum_{i \in I(\mu, \epsilon)} y^{\nu_{i}}$ has non-degenerate critical locus by Lemma 4.7. Taking the quotient of $\mathfrak{t}^{\vee}$ by the span of $\nu_{i}, i \in I(\mu, \epsilon)$ and repeating the computation for the remaining stages implies stage-wise non-degeneracy.

Lemma 4.15. (Wall-crossing for dimensions) Let $X, G, \omega_{t}$ be as above. In the case that $X / /{ }_{t} G$ undergoes a flip with center $Z$ at a singular value $t \in(0, \infty)$, with $t_{ \pm}=t \pm \epsilon$ for $\epsilon$ small and $W_{Z}$ the Landau-Ginzburg potential of $Z$, we have

$$
\begin{aligned}
\operatorname{dim}\left(Q H\left(X / / t_{+} G\right)\right)-\operatorname{dim}\left(Q H\left(X / / t_{-} G\right)\right) & =\operatorname{dim}\left(\Sigma_{t}\right) ! \operatorname{Vol}\left(\Sigma_{t}\right) \operatorname{dim}(Q H(Z)) ; \\
\operatorname{dim}\left(\operatorname{Jac}_{+}\left(W_{X, G, t_{+}}\right)\right)-\operatorname{dim}\left(\operatorname{Jac}_{+}\left(W_{X, G, t_{-}}\right)\right) & =\operatorname{dim}\left(\Sigma_{t}\right) ! \operatorname{Vol}\left(\Sigma_{t}\right) \operatorname{dim}\left(\operatorname{Jac}_{+}\left(W_{Z}\right)\right) .
\end{aligned}
$$

Proof. The first equality in 4.15 concerns the change in dimension of the quantum cohomology. Denote by $\left(\partial \Sigma_{t}\right)_{ \pm}$the union of facets of $\Sigma_{t}$ defined by half-spaces that do not resp. do contain 0 . The corresponding partition of facets determines a partition $\left\{I_{+}, I_{-}\right\}$ 

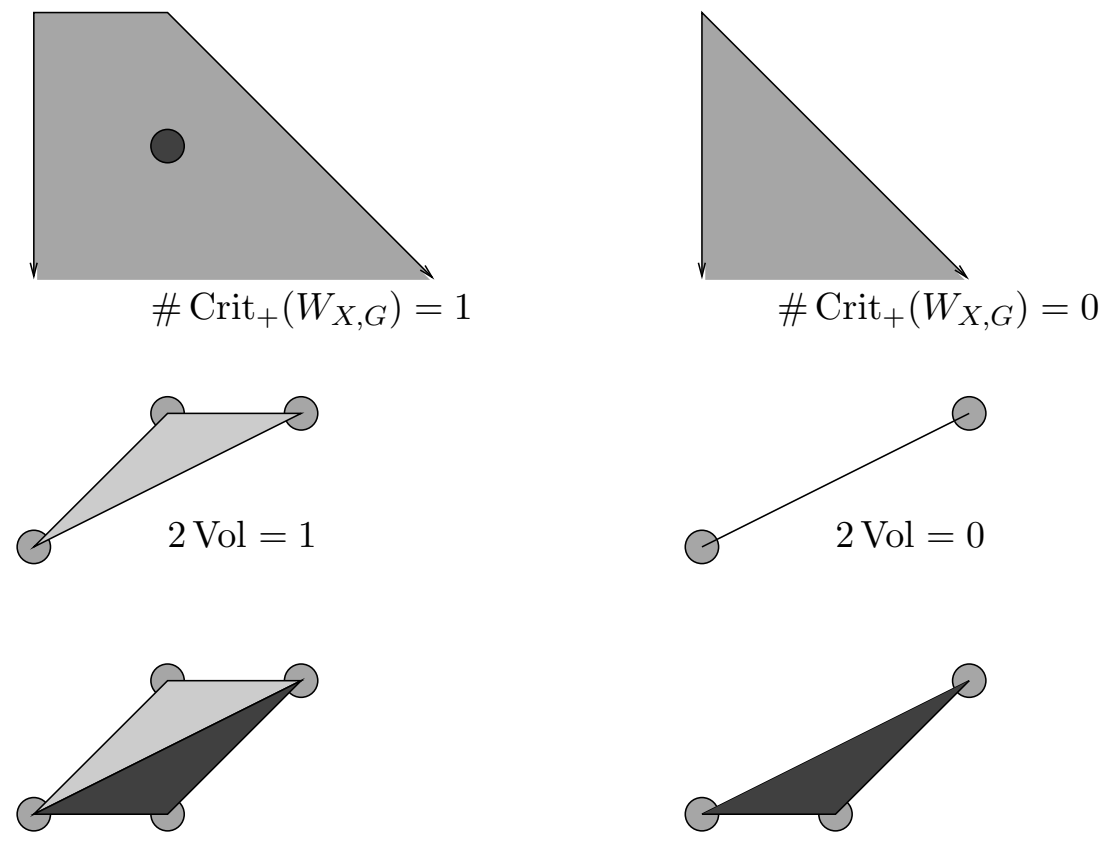

$2 \mathrm{Vol}=2$

$2 \mathrm{Vol}=1$

FiguRE 7. Wall-crossing for dimensions

of $\{1, \ldots, l+1\}$. The morphism of coarse moduli spaces from $X / / t_{ \pm} G$ to $X / / t G$ is a stackyweighted blow-down of the orbit that is the intersection of divisors corresponding to $I_{ \pm}$ onto the center $Z$. Consider the polytope $\Sigma_{t, \pm}$ given as the convex hull of $\left\{\nu_{j}, j \in I_{ \pm}\right\}$ and 0 . The volume of the polytope $\Sigma_{t, \pm}$ is the dimension of the cohomology of a fiber of the inertia stack $I_{E_{ \pm}} \rightarrow Z$ by Lemma 3.11, and $Q H\left(E_{ \pm}\right)=H\left(I_{E_{ \pm}}\right)$by definition. Furthermore, $E_{ \pm}$fibers over $Z$ with fiber a toric stack $E_{ \pm, z}$. The fan of $E_{ \pm, z}$ is the union of cones generated by non-zero vertices in $\Sigma_{t, \pm}$. Hence

$$
\begin{aligned}
\operatorname{dim}\left(Q H\left(E_{ \pm}\right)\right) & =\operatorname{dim}\left(Q H\left(E_{ \pm, z}\right)\right) \operatorname{dim}(Q H(Z)) \\
& =\operatorname{dim}\left(\Sigma_{t, \pm}\right) ! \operatorname{Vol}\left(\Sigma_{t, \pm}\right) \operatorname{dim}(Q H(Z)) .
\end{aligned}
$$

Since $X / / t_{ \pm} G$ are isomorphic away from the exceptional loci,

$$
\begin{aligned}
\frac{\operatorname{dim} Q H\left(X / / t_{+} G\right)-\operatorname{dim} Q H\left(X / / t_{-} G\right)}{\operatorname{dim}(Q H(Z))} & =\operatorname{dim}\left(\Sigma_{t}\right) ! \operatorname{Vol}\left(\Sigma_{t,+}\right)-\operatorname{dim}\left(\Sigma_{t}\right) ! \operatorname{Vol}\left(\Sigma_{t,-}\right) \\
& =\operatorname{dim}\left(\Sigma_{t}\right) ! \operatorname{Vol}\left(\Sigma_{t}\right)
\end{aligned}
$$

which proves the claim.

The second equality in 4.15 describes the change in the dimension of the Jacobian ring. We may suppose that $t_{ \pm}$are sufficiently close to the critical value $t_{j}$ so that there exists a number $c>0$ such that $\Psi\left(\operatorname{Crit}_{+}\left(W_{X, G, t}\right)\right)$ consists of a single value in $(-c, c)$, which crosses the boundary of $\Delta_{X /{ }_{t} G}$ as $t$ crosses $t_{j}$, and the other components of $\Psi\left(\mathrm{Crit}_{+}\left(W_{X, G, t}\right)\right)$ stay outside of $(-c, c)$ for all $t \in\left(t_{-}, t_{+}\right)$. The critical value that crosses 
the boundary corresponds to the intersection of $\operatorname{dim}\left(\Sigma_{t}\right)+1$-hyperplanes varying linearly in $t$. By Kouchnirenko's theorem 3.9 and Proposition 4.13 the number of the critical points $y \in \operatorname{Crit}\left(W_{X, G, t}\right)$ mapping to the singular set in $\Delta_{X / / t}$ is $\operatorname{dim}\left(\Sigma_{t}\right) ! \operatorname{Vol}\left(\Sigma_{t}\right) \operatorname{dim}\left(\operatorname{Jac}_{+}\left(W_{Z}\right)\right)$. For any interval $I \subset \mathbb{R}$, let $\operatorname{Crit}_{I}\left(W_{X, G}\right)$ denote the subset of the critical locus $\operatorname{Crit}\left(W_{X, G}\right)$ consisting of points $y$ with $\inf _{j=1, \ldots, k}\left\langle\Psi(y), \nu_{j}-\omega_{j}\right\rangle \in I$. By the previous paragraph and Propositions 3.9 and 4.13 the difference in the number of critical points of the potential before and after the critical value is equal to

$$
\begin{aligned}
& \operatorname{dim} \operatorname{Jac}_{+}\left(W_{X, G, t_{+}}\right)-\operatorname{dim}_{J_{a c}}\left(W_{X, G, t_{-}}\right)=\left|\operatorname{Crit}_{+}\left(W_{X, G, t_{+}}\right)\right|-\left|\operatorname{Crit}_{+}\left(W_{X, G, t_{-}}\right)\right| \\
& =\left|\operatorname{Crit}_{(c, \infty)}\left(W_{X, G, t_{+}}\right)\right|-\left|\operatorname{Crit}_{(c, \infty)}\left(W_{X, G, t_{-}}\right)\right| \\
& +\left|\operatorname{Crit}_{(0, c)}\left(W_{X, G, t_{+}}\right)\right|-\left|\operatorname{Crit}_{(0, c)}\left(W_{X, G, t_{-}}\right)\right| \\
& =0+\operatorname{dim}\left(\Sigma_{t}\right) ! \operatorname{Vol}\left(\Sigma_{t}\right) \operatorname{dim}\left(\operatorname{Jac}\left(W_{Z}\right)\right)
\end{aligned}
$$

as claimed.

Example 4.16. In Figure 7 we show the flipping simplex for a blow-up of $\mathbb{C}^{2}$ at 0 . More precisely the top figures show the polytope of a blow-up of $\mathbb{C}^{2}$ and of $\mathbb{C}^{2}$ respectively; the image of the critical point under the tropical moment map $\Psi$ is shown as a point in the interior on the upper left. The middle figures show the polytopes $\Sigma_{ \pm}$, spanned by $(-1,0),(0,1),(1,1)$ resp. $(-1,0),(1,1)$ and their volumes $1 / 2$ resp. 0 ; the last figures show the Newton polytopes $\Delta_{X / / t \pm \epsilon}$ of the potentials before and after the blow-down respectively, with the polytopes $\Sigma_{-}$shown as sub-polytope.

Remark 4.17. (a) (Termination of a toric minimal model program) In particular, one sees from the above wall-crossing formula that $\operatorname{dim}\left(Q H\left(X / /{ }_{t} G\right)\right)$ decreases at each wall-crossing. This is one of the proofs of the eventual termination of the toric minimal model program, discussed in [15], [42], where $\operatorname{dim}\left(Q H\left(X / /{ }_{t} G\right)\right)$ is described in combinatorial terms.

(b) (Dependence of the Jacobian ring on the symplectic class) The location of the critical values $\Psi(y), y \in \operatorname{Crit}(W)$ varies with the choice of symplectic class $\omega$, and at certain affine linear hyperplanes (occurring when more than $n+2$ normal vectors have a common value) the critical values $\Psi(y)$ can "collide" as the polarization class $\omega$ varies.

Lemma 4.18. (Equality of dimensions in the Fano case) Suppose that the family $X / /{ }_{t} G$ as above has a unique singular point at $t_{0}$, and undergoes a Mori fibration over a point at $t_{0}$. Then $\operatorname{dim} \mathrm{Jac}_{+}\left(W_{X, G}\right)=\operatorname{dim} Q H\left(X / /{ }_{t} G\right)$.

Proof. In the absence of spurious facets, the Fano case reduces to Kouchnirenko's Theorem 3.9. The assumption that $X / /{ }_{t} G$ undergoes a fibration over a point means that every facet of $\Delta_{X /{ }_{t} G}$ is equidistant from some point $\mu \in \mathfrak{t}_{\mathbb{R}}^{\vee}$, so that $X / /{ }_{t} G$ is Fano. Without loss of generality we may assume that $\mu=0$. Suppose that the presentation of $X$ as a symplectic quotient is the minimal one, that is, each weight of $X$ corresponds to a facet of $\Delta_{X /{ }_{t} G}$ (no spurious facets). In this case all $y \in \mathrm{Crit}_{+}\left(W_{X, G}\right)$ have $\Psi(y)=0$. Thus we may omit the parameters $q$ from the definition of the potential and the number of critical points is equal to $\operatorname{dim} Q H\left(X / /{ }_{t} G\right)$ by Kouchnirenko's Theorem 3.9. 
In the case that the presentation is not minimal, the equality follows from the formal implicit function theorem. Let $W_{X / / G}: \tilde{T}^{\vee}(\Lambda) \rightarrow \Lambda$ denote the Givental potential associated to the minimal presentation. That is, if

$$
\mathcal{T}=\left\{i \mid \operatorname{codim}\left(\left\{\left\langle\nu_{i}, \mu\right\rangle=-\omega_{i}\right\} \cap \Delta_{X / / G}\right)=1\right\} \subset\{1, \ldots, k\}
$$

denotes the indices of the inequalities defining facets of $\Delta_{X / / G}$ then

$$
W_{X / / G}(\tilde{g})=\sum_{i \in \mathcal{T}} q^{\omega_{i}} y_{i}
$$

and each term corresponds to a facet of $\Delta_{X / / G}$. Let $W_{X, G \text {,fake }}=W_{X, G}-W_{X / / G}$ denote the terms arising from the "fake facets", that is, weights of $X$ that do not define facets of $X / / G$ so that

$$
W_{X, G}=W_{X / / G}+W_{X, G, \text { fake }}
$$

Let $c_{1}^{G}(X)_{\min } \in \mathfrak{g}_{\mathbb{Q}}^{\vee} \cong H_{G}^{2}(X)$ be the sum of the weights corresponding to divisors of $X / / G$, that is, the true facets. By the genericity assumption the critical locus $\operatorname{Crit}\left(W_{X / / G}\right)$ is non-degenerate. The formal criterion for smoothness (that is, the formal implicit function theorem as used in the proof of Proposition 4.13) implies that there is an isomorphism $\mathrm{Crit}_{+}\left(W_{X / / G}\right) \rightarrow$ Crit $_{+}\left(W_{X, G}\right)$. That is, adding in the higher order terms give a deformation of the critical locus $\operatorname{Crit}\left(W_{X / / G}\right)$ to $\operatorname{Crit}\left(W_{X, G}\right)$ lying over the interior of the moment polytope $\Delta_{X / / G}$.

By combining the Fano dimensional equality 4.18 and the wall-crossing and fibration formulas 4.15 and 4.11 we have the equality of dimension in general:

Theorem 4.19. (Equality of Dimensions) For $X / / G$ as in the statement of Theorem 1.15, $\operatorname{dim} \mathrm{Jac}_{+}\left(W_{X, G}\right)=\operatorname{dim} Q H(X / / G)$.

Proof. By induction we may assume that the dimensional equality of Theorem 4.19 holds for toric stacks of dimension smaller than $\operatorname{dim}(X / / G)$. By Lemma 4.8 as $t$ varies the toric orbifold $X / / t G$ undergoes a finite sequence of flips or weighted blow-downs $X / / t_{j}-{ }_{\epsilon} G \rightarrow$ $X / / t_{j}+\epsilon G$, followed by a fibration to a toric stack of $\left(X / / t_{n} G\right)^{\prime}$ of smaller dimension with Fano fibers $\left(X / / t_{n} G\right)^{\prime \prime}$. The wall-crossing terms are the same, by Lemma 4.15 . In the case of a fibration, the equality follows from Lemma 4.11.

Proof of Theorems 1.15 and 1.7. By $[50,51,52]$, the linearization $D_{\alpha} \kappa_{X}^{G}$ of the quantum Kirwan map descends to a map

$$
T_{\omega} \kappa_{X}^{G} / Q S R_{X, G}: Q H_{G}(X) / Q S R_{X, G} \rightarrow Q H(X / / G)
$$

is an isomorphism. By Theorem 2.6, $T_{\alpha} \kappa_{X}^{G} / Q S R_{X, G}$ is surjective. By Theorem 4.19, the induced map from $\mathrm{Jac}_{+}\left(W_{X, G}\right)$ to $T_{\kappa_{G}(\omega)} Q H(X / / G)$. Theorem 1.7 follows from the identification with the Jacobian ring in Proposition 3.7.

Corollary 4.20. The quantum cohomology $Q H(X / / G)$ of any proper toric orbifold with projective coarse moduli space $X / / G$ over the universal Novikov field $\Lambda$ is semisimple at bulk deformation $\kappa_{X}^{G}(0)$ for generic symplectic classes $\omega \in H_{G}^{2}(X, \mathbb{Q})$. 
Proof. As explained in [33, Proposition 4.9], semisimplicity at the bulk deformation $\kappa_{X}^{G}(0)$ follows from the identification with the Batyrev ring $\widehat{Q H}_{G}(X) / \widehat{Q S R}_{X}^{G}$, or rather, the Jacobian ring $\mathrm{Jac}_{+}\left(W_{X, G}\right)$ and the fact that for generic $\omega$, the potential $W_{X, G}$ has only stagewise non-degenerate critical values $y \in \operatorname{Crit}(W)$, see Proposition 4.13 and also Iritani [33, Proposition 3.10]. Note that semisimplicity for generic values of $q$ also follows from Lemma 3.10.

Remark 4.21. (a) (Non-semisimple cases) An example of a non-generic symplectic structure with non semi-simple quantum cohomology ring $Q H(X / / G)_{q=1}$ is given in Ostrover-Tyomkin [44].

(b) (Dubrovin conjecture for toric orbifolds) Semisimplicity is related by a conjecture of Dubrovin, see [5], to the existence of a full exceptional collection in the bounded derived category of coherent sheaves $D^{b} \operatorname{Coh}(Y)$ of $Y$. In the toric case the existence of such a collection is proved by Kawamata [37].

(c) (Equivariant first Chern class maps to the potential) Under the isomorphism from $Q H_{G}(X) / Q S R_{X, G}$ to $\operatorname{Jac}\left(W_{X, G}\right)$, the coset of the first Chern class $\left[c_{1}^{G}(X)\right] \in$ $Q H_{G}(X) / Q S R_{X, G}$ maps to the potential $W_{X, G} \in \mathrm{Jac}_{+}\left(W_{X, G}\right)$ itself, by definition of the isomorphism. However, $c_{1}^{G}(X)$ does not map to $c_{1}(X / / G) \in H^{2}(X / / G) \subset$ $Q H(X / / G)$ in general. Consider the example of $G=\mathbb{C}^{\times}$acting on $X=\mathbb{C}$ with weight two, so that $X / / G=\mathbb{C} / / \mathbb{C}^{\times}=\mathbb{P}(2)=B \mathbb{Z}_{2}$ is the stacky half-point. In this case $c_{1}^{G}(X)$ is a degree two class and maps under $D_{\omega} \kappa_{X}^{G}$ to the twisted sector in $X / / G$, since the contributing maps in $\mathcal{M}_{1,1}^{G}(\mathbb{A}, X)$ have degree one. On the other hand, $c_{1}(X / / G)$ is trivial since the tangent bundle is rank zero.

As a corollary to the second part of the Remark and the discussion above we have the following, which "quantifies" the sense in which flips in the minimal model program "make the variety more Fano".

Corollary 4.22. (Decrease in the eigenvalues of $c_{1} \star$ under mmp flips) Suppose that $X /{ }_{t} G$ undergoes a flip at $t=t_{j}$. Then the minimal q-valuation $\min \left(\operatorname{val}_{q}\left(\lambda_{i}\right)\right)$ of the eigenvalues $\lambda_{i} \in \Lambda$ of quantum multiplication by $D_{\omega} \kappa_{X}^{G}\left(c_{1}^{G}(T X)\right)$ on $T_{\kappa_{X}^{G}(\omega)} Q H(X / / G)$ increases.

Proof. The critical values of $W_{X, G}$ moving outside the moment polytope $\Delta_{X / / G}$ at the time $t_{j}$ of the flip are those values $W_{X, G}(y), y \in \operatorname{Crit}\left(W_{X, G}\right)$ with lowest $q$ valuation for $t$ slightly smaller than $t_{j}$. On the other hand, by Remark 4.21 (c) the $q$-valuations of such $y$ are the lowest $q$-valuations of eigenvalues of $D_{\omega} \kappa_{X}^{G} c_{1}^{G}(T X)$

Corollary 4.23. (Equivariant version of the Batyrev presentation) There is a canonical isomorphism $T_{\omega} \widehat{Q H}_{\tilde{G}}(X) / \widehat{Q S R}_{X}^{G, \tilde{G}}(\omega) \rightarrow T_{\kappa_{X}^{\tilde{G}, G}(\omega)} Q H_{\tilde{G} / G}(X / / G)$ for any rational symplectic class $\omega \in H_{\tilde{G}}^{2}(X)$.

Proof. We have already shown in Theorem 1.7 the non-equivariant version of the statement in Corollary 4.23, that is, setting the equivariant parameters for $T=\tilde{G} / G$ to zero. By equivariant formality, $Q H_{\tilde{G} / G}(X / / G)$ is a free $Q H_{\tilde{G} / G}(\mathrm{pt})$-module, and this implies 
that $\widehat{Q H}_{\tilde{G} / G}(X / / G)$ is a free $\widehat{Q H}_{\tilde{G} / G}(\mathrm{pt})$ module. Since the same is true for the lefthand-side, it follows that the linearization of the equivariant quantum Kirwan map map $T_{\omega} \widehat{Q H}_{\tilde{G}}(X) / \widehat{Q S R}_{X}^{G, \tilde{G}} \rightarrow T_{\kappa_{X}^{\tilde{G}, G}(\omega)} Q H_{\tilde{G} / G}(X / / G)$ is also an isomorphism.

4.1. Invariance of quantum cohomology under weighted toric flops. In this section we digress to show that quantum cohomology is invariant under weighted toric flops. Let $X / /{ }_{t} G$ be a family of toric quotients as above (not necessarily in the direction of the canonical class $\left.c_{1}(X / / t G)\right)$ so that $X / /{ }_{t} G$ is a locally free quotient for $t$ generic.

Definition 4.24. The variation of git quotient $X / / t G$ undergoes a flop at a singular time $t_{i}$ if there is a unique point $x \in X$ semistable for $t_{i}$ with positive-dimensional stabilizer $G_{x}$, the group $G_{x}$ is one-dimensional and the sum $\sum_{i=1}^{l} \nu_{i}$ of the weights $\nu_{i} \in \mathbb{Z}, i=1, \ldots, l$ for $G_{x}$ on $T_{x} X$ is zero.

Lemma 4.25. If $X / /{ }_{t} G$ undergoes a flop at $t_{i}$ then the quotients $X / / t_{i} \pm \epsilon$ on either side of the critical value $t_{i}$ are $K$-equivalent in the sense that the canonical bundles are pull-backs of the same (rational) bundle under the morphism of coarse moduli spaces $X / / t_{i} \pm \epsilon \rightarrow$ $X / / t_{i} G$.

Proof. By Kempf's descent criterion, see [18, 2.3], the canonical bundle $K_{X}$ descends to the singular quotient $X / / t_{i} G$ and similarly $K_{X}$ descends to the canonical bundle $K_{X / / t_{i} \pm \epsilon} G$ on $X / / t_{i} \pm \epsilon G$; where the quotients are stacks these bundles exist rationally on the corresponding moduli spaces. The maps $X / / t_{i} \pm \epsilon G \rightarrow X / / t_{i} G$ are induced by inclusions of semistable loci and the claim follows.

Proposition 4.26. If $X / /{ }_{t} G$ undergoes a flop at $t=t_{i}$ then the quantum cohomologies $Q H\left(X / / t_{i} \pm \epsilon G\right)$ are isomorphic as vector spaces, and the quantum products $\star_{t}$ are related by analytic continuation.

Proof. It suffices to show, by the description of the quantum cohomology in Theorem 1.15 that no critical values cross the boundary of the moment polytope at the critical time. We suppose that the facets $F_{j, t_{i} \pm \epsilon} \subset \Delta\left(X / / t_{i} \pm \epsilon G\right)$ from Definition (4.6) meeting the singular moment value (which we may assume maps to 0 ) at time $t_{i}$ (which we may assume equals 0 ) are numbered $1, \ldots, l+1$. By the genericity assumption in Proposition 4.5 and Lemma 4.14, the critical values $y_{t} \in \operatorname{Crit}\left(W_{t}\right)$ for each $t$ mapping to 0 at $t=0$ are stagewise non-degenerate. By the analysis in Lemma 4.13, each such $y_{t}$ maps under $\Psi$ to a point $\mu t \in \mathfrak{t}^{\vee}$ such that $\left\langle\mu t, \nu_{i}\right\rangle-c_{i} t, i=1, \ldots, l+1$ are independent of $i$. By local triviality of the canonical bundle, the divisor $\sum_{i=1}^{l+1} c_{i} D_{i}$ with coefficients $c_{i}$ is locally linearly equivalent to the divisor $\sum_{i=1}^{l+1}\left(c_{i}-c\right) D_{i}$ with coefficients $c_{i}-c$, for any constant $c$. Thus for any $c \in \mathbb{R}$ there exists a $\mu^{\prime} \in \mathfrak{t}_{\mathbb{R}}^{\vee}$ such that $\left\langle\mu^{\prime} t, \nu_{i}\right\rangle=\left(c_{i}-c\right) t, i=1, \ldots, n+1$ is independent of $i$. Taking e.g. $c_{1}=c$ we obtain that $\left\langle\mu^{\prime}, t \nu_{i}\right\rangle=0$ for all $i=1, \ldots, l+1$ vanish for all $i$, and the inequalities $\left\langle\mu^{\prime} t, \nu_{i}\right\rangle \geq\left(c_{i}-c\right) t$ are satisfied with equality for all $t$. Thus the family $\mu^{\prime} t$ does not cross the boundary of the moment polytope. It follows that $\operatorname{dim} \mathrm{Jac}_{+}\left(W_{X / / t} G\right)$ is independent of $t$ in a neighborhood of $t_{i}$, so that $\mathrm{Jac}_{+}\left(W_{X / / t_{i} \pm \epsilon}\right)$ are isomorphic as vector 
spaces. The products $\star_{t}$ are related by analytic continuation by analytic dependence of the Jacobian ideal $\left\langle\partial_{\lambda} W_{X, G}\left(y e^{\lambda}\right)_{\lambda=0}\right\rangle$ in Definition 1.13 on the symplectic class.

\section{Minimal models and nON-Displaceable Lagrangians}

This section is a discussion of how the results here combine with those of [53], [49] on non-displaceable Lagrangian tori. In particular we explain that toric orbifolds can have infinitely many non-displaceable tori because they can have infinitely many runnings of the toric minimal model program. Recall the following from Fukaya-Oh-Ohta-Ono [20], [19, Theorem 3.17,Corollary 4.6] and Woodward [53]:

Theorem 5.1. (Non-displaceable toric moment fibers via critical points of the Givental potential) Let $X$ be a finite dimensional vector space with a linear action of a torus $G$ and polarization so that the git quotient $X / / G$ is a proper toric Deligne-Mumford stack with projective coarse moduli space, moment map $\Phi: X / / G \rightarrow \mathfrak{t}_{\mathbb{R}}^{\vee}$ and moment polytope $\Delta_{X / / G}=\Phi(X / / G)$. Let $W_{X, G}$ denote the Givental potential and $\Psi:$ Crit $_{+}\left(W_{X, G}\right) \rightarrow \Delta_{X / / G}$ the tropical moment map. Then for any $y \in \mathrm{Crit}_{+}\left(W_{X, G}\right)$, the inverse image $\Phi^{-1}(\Psi(y)) \subset$ $X / / G$ is a Hamiltonian non-displaceable Lagrangian torus.

The proof in [53] uses that non-displaceability of a Lagrangian in $X / / G$ is implied by the $G$-non-displaceability of its pre-image in $X$, and this non-displaceability is governed by a suitable $G$-equivariant version of Floer homology.

5.1. Generic tmmp runnings. By the results of the previous sections, we may understand the critical values of the potential in terms of the corresponding minimal model program. Let $Y$ be a smooth proper toric Deligne-Mumford stack with polarized projective coarse moduli space, and $G$ a torus acting on a vector space $X$ so that $X / / G$, equipped with its residual torus action, is isomorphic to $Y$.

Notation 5.2. $\quad$ (a) (Toric minimal model program) The sequence of stacks

$$
X / /{ }_{t} G, t \in[0, \infty), \quad Y=X / / G=X / /{ }_{0} G
$$

obtained by varying the equivariant symplectic class $\omega$ in the direction of $-c_{1}^{G}(X)$ will be called a toric minimal model program (tmmp) running for $Y$. Our terminology differs from the standard terminology in that we include the path $\omega_{t}$ of symplectic classes in the definition of the running.

(b) (Transition times) The values $t_{1}, \ldots, t_{n}$ of $t$ for which $X / /{ }_{t} G$ is singular (that is, there exist points $x$ in the stable locus $X^{\mathrm{ss}}$ with infinite stabilizer subgroups $G_{x}$ ) are the transition times for the tmmp running.

(c) (Dimension jumps) Let $t_{j, \pm}=t_{j} \pm \epsilon$ for $\epsilon$ sufficiently small so that $t_{j-1}+\epsilon<$ $t_{j}-\epsilon, j=2, \ldots, n$. Let

$$
d_{j}=\operatorname{dim} Q H\left(X / / t_{j,-} G\right)-\operatorname{dim} Q H\left(X / / t_{j,+} G\right)
$$

denote the dimension jump at $t_{j}$. 
(d) (Singular moment values) For simplicity, we assume that $X / / t_{j} G$ has a connected singular set with infinite stabilizer subgroups $\left(X / / t_{j} G\right)^{\operatorname{sing}}$ mapping to singular moment value

$$
\Phi\left(\left(X / / t_{j} G\right)^{\text {sing }}\right) \subset \Delta_{X / t_{j} G} \subset \Delta_{X / / 0} G, \quad j=1, \ldots, n .
$$

(e) (Fiber of the Fano fibration) Suppose furthermore that for $t$ just before $t_{n}$, the quotient $X / /{ }_{t} G$ is a fibration over $Y^{\prime \prime}=\left(X / / t_{n} G\right)^{\prime \prime}$, with Fano fiber $Y^{\prime}=\left(X / / t_{n} G\right)^{\prime}$.

Remark 5.3. (a) (Non-uniqueness of tmmp runnings) Many presentations of $X / / G$ as a git quotient will give the same tmmp runnings. However, toric orbifolds can have infinitely many tmmps runnings, corresponding to different realizations of $X / / G$ as git quotients. (Recall we take the family of symplectic class $\omega_{t}$ as part of the definition of the tmmp running.) The "fake facet equalities" $\left\langle\cdot, \mu_{j}\right\rangle=\omega_{t, j}$ (those with empty solution set in $\Delta_{X / G}$ ) can "catch up" to the "true facets" (those with non-empty solution set) under the deformation $\omega_{t}$ at arbitrary times. For example, taking the minimal presentation of $\mathbb{P}(1,3,5)$ as a git quotient $\mathbb{C}^{3} / / \mathbb{C}^{\times}$yields a trivial toric mmp, but introducing a presentation as a quotient $\mathbb{C}^{4} / /\left(\mathbb{C}^{\times}\right)^{2}$ yields a toric mmp with a flip to an "orbifold Hirzebruch surface", which is similar to the example discussed in [49]. See Figure 8. Since in this case the time of the transition depends on the position of the extra spurious facet (shown as the rightmost dotted line in Figure 8) this give an example with infinitely many tmmp runnings. The computation Abreu-Borman-McDuff [2, Proposition 4.1.4] show that in the manifold case there is a unique tmmp, since the fake facets never "catch up".

(b) (Induced tmmp running for fibrations) Any presentation of $Y$ as a git quotient $X / / G$ induces a presentation of the base $\left(X / / t_{n} G\right)^{\prime \prime}$ of the final fibration as a git quotient, corresponding to the inequalities that are not defining inequalities for the Fano fiber $\left(X / / t_{n} G\right)^{\prime \prime}$, that is, the inequalities which become strict equalities for the final polytope $\Delta_{X / t_{n} G}$. Hence, any presentation of $Y$ induces a tmmp running for the base of the final fibration.

Notation 5.4. (Eigenspace decomposition for quantum multiplication by $c_{1}$ ) Given a presentation $\mathcal{P}=(X, G)$ of a toric stack $X / / G$ as a git quotient let $\omega_{\mathcal{P}}=\kappa_{X}^{G}(\omega)$ and

$$
c_{1}(Y, \mathcal{P}):=D_{\omega} \kappa_{X}^{G} c_{1}^{G}(X) \in T_{\omega_{\mathcal{P}}} Q H(Y) .
$$

Consider the decomposition of quantum cohomology into eigenspace sums for quantum multiplication by $c_{1}(Y, \mathcal{P})$ :

$$
T_{\omega_{\mathcal{P}}} Q H(Y) \cong \bigoplus_{j=1}^{n} T_{\omega_{\mathcal{P}}} Q H(Y)_{\mathcal{P}, j} .
$$

where each $Q H(Y)_{\mathcal{P}, j}$ is a sum of eigenspaces for eigenvalue $\lambda$ with the same $q$-valuation $\operatorname{val}_{q}(\lambda)$.

Theorem 5.5. (Relationships between tmmps and quantum cohomology) Let $Y$ be a compact toric orbifold and $\mathcal{P}$ be a generic toric $\mathrm{mmp}$ for $Y$ obtained from a quotient 

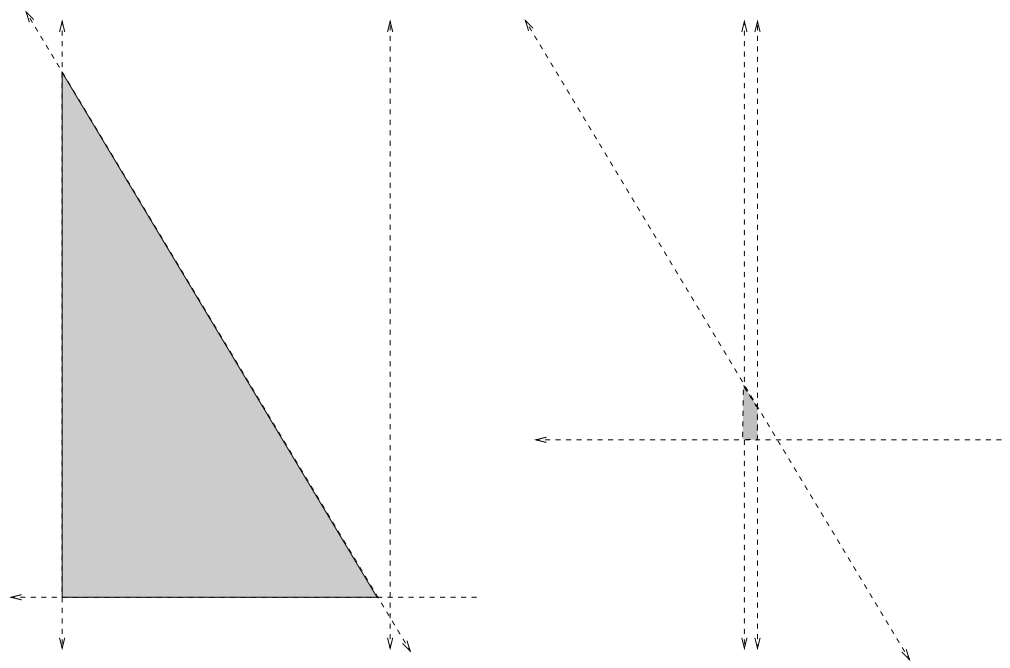

FiguRe 8 . Non-trivial toric mmp for $\mathbb{P}(1,3,5)$

presentation $Y=X / / G$ with transition times $t_{j}$, dimension jumps $d_{j}$ and singular moment values $\psi_{j} \in \Psi\left(\operatorname{Crit}\left(W_{X, G}\right)\right), j=1, \ldots, n$.

(a) The transition times $t_{j}, j=1, \ldots, n$ of the tmmp running are the q-valuations of the eigenvalues $\lambda_{j}$ of quantum multiplication by $c_{1}(Y, \mathcal{P})$.

(b) The dimension jumps $d_{j}, j=1, \ldots, n$ of the tmmp running at time $t_{j}, j=1, \ldots, n$ are the dimensions of the corresponding eigenspaces,

$$
\operatorname{dim} T_{\omega_{\mathcal{P}}} Q H(Y)_{\mathcal{P}, j}=d_{j}
$$

(c) for each $j=1, \ldots, n-1$, the inverse image $L_{j}$ of $\psi_{j}$ in $Y$ is Hamiltonian nondisplaceable. The number of local systems making the Lagrangian Floer homology of $L_{j}$ non-vanishing, counted with multiplicity, is also equal to $d_{j}$;

(d) for $j=n$, the factor $T_{\omega_{\mathcal{P}}} Q H(Y)_{\mathcal{P}, n}$ further splits

$$
T_{\omega_{\mathcal{P}}} Q H(Y)_{\mathcal{P}, n}=\bigoplus_{j=1}^{n_{1}} T_{\omega_{\mathcal{P}}} Q H(Y)_{\mathcal{P}, n, j}
$$

$$
\operatorname{dim} T_{\omega_{\mathcal{P}}} Q H(Y)_{\mathcal{P}, n, j}=\operatorname{dim}\left(T_{\omega_{\mathcal{P}^{\prime}}} Q H\left(Y^{\prime}\right)\right) d_{j, 1}
$$

according to a splitting induced from a tmmp running $\mathcal{P}_{1}$ for the base $Y^{\prime \prime}=$ $\left(X / / t_{n} G\right)^{\prime \prime}$ and fiber $Y^{\prime}=\left(X / / t_{n} G\right)^{\prime}$ with dimension jumps $d_{j, 1}$ as in Remark 5.3 (b), and for each singular value $\psi_{j, 1}$ in such a tmmp running the inverse image in $Y$ is Hamiltonian non-displaceable.

Proof. Suppose that $\mathcal{P}$ is a minimal model program corresponding to a presentation of $Y$ as a quotient of $X$ by $G$. By the main result Theorem $1.15 Q H(Y) \cong \mathrm{Jac}_{+}\left(W_{X, G}\right)$, and the latter admits a decomposition into components corresponding to critical points with fixed value of the tropical moment map from Definition 3.14. Quantum multiplication by $c_{1}(Y, \mathcal{P})=D_{\omega} \kappa_{X, G}\left(c_{1}^{G}(X)\right)$ is given by multiplication by $W_{X, G}$ itself, hence (a) and (b). 
By Lemma 4.15, each summand has dimension that of the dimension jump in the given tmmp running. (c) is Theorem 5.1, with the multiplicity computed using Kouchnirenko's theorem. (d) is a consequence of Lemma 4.11 and Theorem 5.1.

5.2. Non-generic tmmp runnings. The results above are for generic initial symplectic class only. Abreu has pointed out to us that there is still a connection between nondisplaceable Lagrangians and minimal model programs, even in the case that minimal model program involves flips over "singular" toric orbifolds, in the sense that the critical points of the Landau-Ginzburg potential for the singular toric manifolds "cause" nondisplaceable moment fibers in the original manifold or orbifold. More precisely, we suppose that the generic stabilizer is trivial and we are in the following situation:

Notation 5.6. $\quad$ (a) (Singular base of a tmmp transition) Let $Y$ be a compact toric orbifold with symplectic class $\omega_{Y}$. Consider a toric mmp for $Y$ with dimension jumps $d_{j}$ and singular moment values $\psi_{j}, j=1, \ldots, n$, and the flip/contraction at time $t_{j}$ has base a possibly singular toric variety $Z_{j}$ with polytope $\Delta_{j}$. Let $\mathfrak{t}_{j}^{\vee}$ denote the span of $\Delta_{j}$ and $T_{j}^{\vee} \subset T^{\vee}$ the torus with Lie algebra $\mathfrak{t}_{j}^{\vee}$.

(b) (Normal part of the potential) Let

$$
W_{X, G, j}: T^{\vee}\left(\Lambda_{0}\right) \rightarrow \Lambda, \quad y \mapsto \sum_{\left.\mathrm{d} \nu_{j}\right|_{\Delta_{j}}=0} q^{\omega_{j}} y^{\nu_{j}}
$$

denote the part of the potential $W_{X, G}$ corresponding to the normal vectors constant on $\Delta_{j}$. Thus

$$
W_{X, G}=W_{X, G, j}+W_{X, G, j}^{\prime}
$$

where $W_{X, G, j}^{\prime}$ is the sum of terms corresponding to vector $\nu_{j}$ that are non-constant on $\Delta_{j}$.

(c) (Normally non-degenerate) We say $W_{X, G}$ is normally non-degenerate at $\Delta_{j}$ if each critical point $y \in \operatorname{Crit}\left(W_{X, G, j}\right)$ is non-degenerate. An example is shown in Figure 9 .

Theorem 5.7. (Non-displaceable Lagrangians via non-generic tmmps) Suppose that $Y, \mathcal{P}$ are as above so that $W_{X, G}$ is normally non-degenerate at $\Delta_{j}$, with leading order terms of order $q^{\alpha}$. Then each critical point $\left(y^{\prime \prime}, y^{\prime}\right)$ of $W_{X, G, j} \times W_{X, G, j}^{\prime}$ with $\operatorname{val}_{q}\left(y^{\prime \prime}\right)>\alpha$ is equivalent, modulo terms vanishing on $T_{j}$, to a critical point $y$ of $W_{X, G}$, and so $\Psi_{j}(y)$ defines a non-displaceable moment fiber in $Y$.

Proof. The proof is an order-by-order correction argument, using Proposition 4.13, see also the implicit function theorem of Fukaya et al [20, Theorem 10.4]. Note that the tropical moment map $\Psi_{j}: \operatorname{Crit}\left(W_{X, G, j}\right) \rightarrow \operatorname{int}\left(\Delta_{j}\right)$ maps to $\Delta$ via the inclusion $\Delta_{j} \rightarrow \Delta$. Any lift of a critical point $y \in \operatorname{Crit}\left(W_{X, G, j}\right) \subset T_{j}^{\vee}$ has the property that $\mathrm{d} W_{X, G}(y)$ descends to $\mathfrak{t}^{\vee} / \mathfrak{t}_{j}^{\vee}$, since the partial derivatives in the direction of $\mathfrak{t}_{j}^{\vee}$ vanish. As in 4.13, [20, Theorem 10.4], the point $y^{\prime \prime} y^{\prime} \in T$ may be corrected by an element of $T^{\vee} / T_{j}^{\vee}$ to a critical point $y$ of the full potential $W_{X, G}$. It follows from [19, Theorems 3.19,Corollary 4.6] that these fibers have non-trivial Floer cohomology, and so are non-displaceable. 


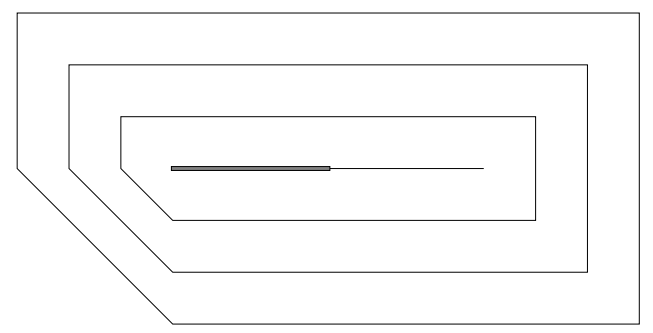

FiguRE 9. A singular minimal model program

Example 5.8. The following example was pointed out to us by M. Abreu. Suppose that $Y$ is the toric manifold whose polytope has vertices $(0,1),(0,2),(1,0),(4,0),(4,2)$, which is a blow-up of a product $\mathbb{P}^{1} \times \mathbb{P}^{1}$; this was the first example, discovered in FukayaOh-Ohta-Ono [20], of a toric manifold with a continuum of non-displaceable toric fibers. The minimal model program for this symplectic class has no flips, and the final step is a "singular fibration" over a $\mathbb{P}^{1}$, with a single singular fiber consisting of a nodal $\mathbb{P}^{1}$. We suppose that $X / / G$ has a non-minimal presentation as a git quotient, so that there is a spurious facet of $\Delta_{X / / G}$ with equation $\lambda_{1}=-\epsilon$ for $\epsilon>0$. The potential $\left.W_{X, G, 1}^{\prime}\right|_{t_{1}^{\vee}}$ for this base, allowing bulk deformations at the divisors with normal vectors $(1,0)$ and $(1,1)$, is of the form $W_{X, G, 1}^{\prime}\left(y_{1}, y_{2}\right)=y_{1}+y_{1} y_{2}+q^{4} / y_{1}+q^{-\epsilon} y_{1}$ while $W_{X, G, 1}\left(y_{2}\right)=y_{2}+q^{2} / y_{2}$. Therefore

$$
W_{X, G, 1}^{\prime}(\cdot,-1)=q^{4} / y_{1}+q^{-\epsilon} y_{1}
$$

has critical points, as $\epsilon$ varies, given by $\left(y_{1},-1\right)$ with $\Psi\left(y_{1},-1\right)=(-\infty, 2)$. Those with $\operatorname{val}_{q}\left(y_{1}\right)>1$ can be corrected by Theorem 5.7 to honest critical points. So the toric moment fibers above the segment between $(1,1)$ and $(2,1)$ are Hamiltonian non-displaceable. Nondisplaceability of the end-points $(1,1)$ and $(2,1)$ holds by continuity. This reproduces the non-displaceability result in this case from [20]. See Figure 9.

Remark 5.9. If $X / / G$ is non-compact, a minimal model program for $X / / G$ may have no transitions. For example, suppose that $X / / G$ is the total space of $\mathcal{O}_{\mathbb{P}^{1}}(-d)$. The moment polytope of $X / / G$ is $\Delta_{X / / G}=\left\{\mu_{2} \geq 0, \mu_{1}+\mu_{2} \geq-d / 2,-\mu_{1}+\mu_{2} \geq-d / 2\right\}$. For $d=2$, the resulting running of the minimal model program has $\Delta_{X / t} G$ a family of translations of $\Delta_{X / / G}$. For $d>2$, the minimal model program running $X / /{ }_{t} G$ corresponds to translation of the polytope $\Delta_{X / / t} G$ together with a dilation $\mathfrak{t}_{\mathbb{R}}^{\vee} \rightarrow \mathfrak{t}_{\mathbb{R}}^{\vee}$ by a constant greater than 1 .

\section{REFERENCES}

[1] D. Abramovich, T. Graber, and A. Vistoli. Gromov-Witten theory of Deligne-Mumford stacks. Amer. J. Math., 130(5):1337-1398, 2008.

[2] M. Abreu, M. Strom Borman, and D. McDuff. Displacing Lagrangian toric fibers by extended probes. arxiv:1203.1074.

[3] M. F. Atiyah. Angular momentum, convex polyhedra and algebraic geometry. Proc. Edinburgh Math. Soc. (2), 26(2):121-133, 1983.

[4] V. V. Batyrev. Quantum cohomology rings of toric manifolds. Astérisque, (218):9-34, 1993. Journées de Géométrie Algébrique d'Orsay (Orsay, 1992).

[5] A. Bayer. Semisimple quantum cohomology and blowups. Int. Math. Res. Not., (40): 2069-2083, 2004. 
[6] L. Bonavero and M. Brion. Geometry of toric varieties. Société Mathématique de France, 2002.

[7] L. A. Borisov, L. Chen, and G. G. Smith. The orbifold Chow ring of toric Deligne-Mumford stacks. J. Amer. Math. Soc., 18(1):193-215 (electronic), 2005.

[8] J. Brown. Gromov- Witten Invariants of Toric Fibrations. Int. Math. Res. Not. IMRN 19:5437-5482, 2014. arxiv:0901.1290.

[9] K. Chan, S.-C. Lau, N. Conan Leung, and H.-H. Tseng. Open Gromov-Witten invariants and mirror maps for semi-Fano toric manifolds. arxiv:1112.0388.

[10] W. Chen and Y. Ruan. Orbifold Gromov-Witten theory. In Orbifolds in mathematics and physics (Madison, WI, 2001), volume 310 of Contemp. Math., pages 25-85. Amer. Math. Soc., Providence, RI, 2002.

[11] T. Coates, Y.-P. Lee, A. Corti, and H.-H. Tseng. The quantum orbifold cohomology of weighted projective spaces. Acta Math., 202(2):139-193, 2009.

[12] T. Coates, A. Corti, H. Iritani, H.-H. Tseng. A Mirror Theorem for Toric Stacks. Compos. Math. 151 (2015), no. 10, 1878-1912. arxiv:1310.4163.

[13] T. Coates, A. Corti, H. Iritani, H.-H. Tseng. Hodge-Theoretic Mirror Symmetry for Toric Stacks. arxiv:1606.07254.

[14] D. A. Cox and S. Katz. Mirror symmetry and algebraic geometry, volume 68 of Mathematical Surveys and Monographs. American Mathematical Society, Providence, RI, 1999.

[15] D. A. Cox, J. B. Little, and H. K. Schenck. Toric varieties, volume 124 of Graduate Studies in Mathematics. American Mathematical Society, Providence, RI, 2011.

[16] V. I. Danilov. The geometry of toric varieties. Uspekhi Mat. Nauk, 33(2(200)):85-134, 247, 1978.

[17] I. V. Dolgachev and Yi Hu. Variation of geometric invariant theory quotients. Inst. Hautes Études Sci. Publ. Math., (87):5-56, 1998. With an appendix by Nicolas Ressayre.

[18] J.-M. Drezet and M. S. Narasimhan. Groupe de Picard des variétés de modules de fibrés semi-stables sur les courbes algébriques. Invent. Math., 97(1):53-94, 1989.

[19] K. Fukaya, Y.-G. Oh, H. Ohta, and K. Ono. Lagrangian Floer theory on compact toric manifolds II : Bulk deformations. Selecta Math., (17): 609-711, 2011.

[20] K. Fukaya, Y.-G. Oh, H. Ohta, and K. Ono. Lagrangian Floer theory on compact toric manifolds. I. Duke Math. J., 151(1):23-174, 2010.

[21] K. Fukaya, Y.-G. Oh, H. Ohta, and K. Ono. Lagrangian floer theory and mirror symmetry on compact toric manifolds. Lagrangian Floer theory and mirror symmetry on compact toric manifolds. Astérisque $376,2016$. arxiv:1009.1648.

[22] W. Fulton. Intersection theory. Springer-Verlag, Berlin, second edition, 1998.

[23] A. B. Givental. Equivariant Gromov-Witten invariants. Internat. Math. Res. Notices, (13):613-663, 1996.

[24] A. Givental. A mirror theorem for toric complete intersections. In Topological field theory, primitive forms and related topics (Kyoto, 1996), volume 160 of Progr. Math., pages 141-175. Birkhäuser Boston, Boston, MA, 1998.

[25] A. Givental. Homological geometry and mirror symmetry. In Proceedings of the International Congress of Mathematicians, Vol. 1, 2 (Zürich, 1994), pages 472-480, Basel, 1995. Birkhäuser.

[26] E. González and H. Iritani. Seidel elements and Mirror transformations. Selecta Math. (N.S.) 18:557590, 2012. arxiv:1103.4171.

[27] E. González, P. Solis, and C. Woodward. Properness for scaled gauged maps. J. Algebra, 490:104-157, 2017.

[28] T. Graber and R. Pandharipande. Localization of virtual classes. Invent. Math., 135(2):487-518, 1999.

[29] A. Grothendieck. Éléments de géométrie algébrique. III. Étude cohomologique des faisceaux cohérents. I. Inst. Hautes Études Sci. Publ. Math., (11):167, 1961, (17):91, 1963.

[30] M. A. Guest. Quantum cohomology via D-modules. Topology, 44(2):263-281, 2005.

[31] K. Hori and C. Vafa. Mirror symmetry. hep:th/0002222.

[32] H. Iritani. Quantum D-modules and generalized mirror transformations. Topology, 47(4):225-276, 2008. 
[33] H. Iritani. An integral structure in quantum cohomology and mirror symmetry for toric orbifolds. Adv. Math., 222(3):1016-1079, 2009.

[34] H. Iritani. Convergence of quantum cohomology by quantum Lefschetz. J. Reine Angew. Math., 610:29-69, 2007.

[35] J. Jurkiewicz. Chow ring of projective nonsingular torus embedding. Colloq. Math., 43(2):261-270 (1981), 1980.

[36] J. Jurkiewicz. Torus embeddings, polyhedra, $k^{*}$-actions and homology. Dissertationes Math. (Rozprawy Mat.), 236:64, 1985.

[37] Y. Kawamata. Derived categories of toric varieties. Michigan Math. J., 54(3):517-535, 2006.

[38] F. C. Kirwan. Cohomology of Quotients in Symplectic and Algebraic Geometry, volume 31 of Mathematical Notes. Princeton Univ. Press, Princeton, 1984.

[39] A. G. Kouchnirenko. Polyèdres de Newton et nombres de Milnor. Invent. Math., 32(1):1-31, 1976.

[40] E. Lerman. Symplectic cuts. Math. Res. Letters, 2:247-258, 1995.

[41] E. Mann. Orbifold quantum cohomology of weighted projective spaces. J. Algebraic Geom., 17(1):137$166,2008$.

[42] K. Matsuki. Introduction to the Mori program. Universitext. Springer-Verlag, New York, 2002.

[43] D. McDuff and S. Tolman. Topological properties of Hamiltonian circle actions. IMRP Int. Math. Res. Pap., pages 72826, 1-77, 2006

[44] Y. Ostrover and I. Tyomkin. On the quantum homology algebra of toric Fano manifolds. Selecta Math. (N.S.), 15(1):121-149, 2009.

[45] M. Reid. Decomposition of toric morphisms. In Arithmetic and geometry, Vol. II, volume 36 of Progr. Math., pages 395-418. Birkhäuser Boston, Boston, MA, 1983.

[46] H. Spielberg. The Gromov-Witten invariants of symplectic toric manifolds, and their quantum cohomology ring. C. R. Acad. Sci. Paris Sér. I Math., 329(8):699-704, 1999.

[47] M. Thaddeus. Geometric invariant theory and flips. J. Amer. Math. Soc., 9(3):691-723, 1996.

[48] H.-H. Tseng and D. Wang. Seidel Representations and Quantum Cohomology of Toric Orbifolds. arxiv:1211.3204.

[49] G. Wilson and C. Woodward. Quasimap Floer cohomology and singular symplectic quotients. arXiv:1105.0712.

[50] Chris T. Woodward. Quantum Kirwan morphism and Gromov-Witten invariants of quotients I. Transformation Groups 20 (2015) 507-556. arXiv:1204.1765.

[51] Chris T. Woodward. Quantum Kirwan morphism and Gromov-Witten invariants of quotients II. Transformation Groups 20 (2015) 881-920. arXiv:1408.5864.

[52] Chris T. Woodward. Quantum Kirwan morphism and Gromov-Witten invariants of quotients III. Transformation Groups 20 (2015) 1155-1193. arXiv:1408.5869.

[53] C. Woodward. Gauged Floer theory of toric moment fibers. Geom. and Func. Anal., 21:680-749, 2011.

[54] C. Woodward and G. Xu. An open quantum Kirwan map. arXiv:1806.06717.

Department of Mathematics University of Massachusetts Boston 100 William T. MorrisSEy Boulevard Boston, MA 02125

E-mail address: eduardo@math.umb.edu

Mathematics-Hill Center, Rutgers University, 110 Frelinghuysen Road, Piscataway, NJ 08854-8019, U.S.A.

E-mail address: woodwardc@gmail.com 


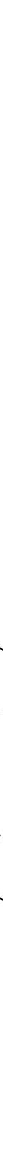

